\title{
HIGH RESOLUTION NUCLEAR MAGNETIC RESONANCE SPECTROSCOPY FOR LAB-ON-A-CHIP DEVICES VIA INDUCTIVE COUPLING
}

\author{
A Thesis Presented to the \\ Faculty of the School of Engineering and Applied Science \\ UNIVERSITY OF VIRGINIA \\ In Partial Fulfillment \\ of the requirements for the \\ Degree of Master of Science in the \\ Department of Mechanical and Aerospace Engineering
}

by

Herbert Ryan

August 2013 
APPROVAL SHEET

The thesis

is submitted in partial fulfillment of the requirements

for the degree of

Master of Science

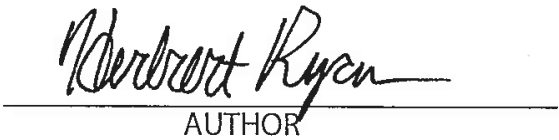

The thesis has been read and approved by the examining committee:

Marcel Utz

Advisor

Hossein Haj-Hariri

David S. Cafiso

Accepted for the School of Engineering and Applied Science:

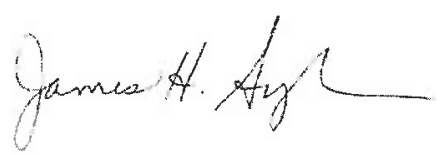

Dean, School of Engineering and Applied Science

August

2013 


\begin{abstract}
The goal of this work is to create a integrated microcoil "lab-on-a-chip" (LOC) device for inductively coupled Nuclear Magnetic Resonance spectroscopy, with sufficient sensitivity and spectral resolution such that metabolomic analysis, for small sample volumes in a wide range of applications, becomes feasible.

Much excitement has surrounded the field of microfluidic LOC devices for reasons of cost and high-throughput capability. These devices combine reagents in a miniaturized chemical reactor network to sort, process, and ultimately analyze a desired sample. Fabrication in glass or polymer substrates via lithographic methods, similar to those used in microelectronics, make LOC devices very cheap and expendable. In contrast to this emerging field is Nuclear Magnetic Resonance (NMR) spectroscopy which is an established tool, ranging in applicability from protein structure determination to non-invasive whole body imaging (called Magnetic Resonance Imaging (MRI) in practice). Although in principle, NMR possesses the high specificity needed for a microfluidic analytical tool, in practice there are several limitations relating to sensitivity and resolution. This thesis reports on methods to integrate NMR spectroscopy with microfluidic devices without sacrificing sensitivity.
\end{abstract}

Theoretical aspects of NMR are explored in regards to applicability of this method to the small sample regime. Further, microcoil fabrication and integration work is presented for inductively coupled coils. Planar spiral resonators are fabricated lithographically by etching $\mathrm{Cu}$, laminated on polyimide. Coil performance is characterized by both magnetic resonance images and spectroscopy. A single-scan limit of detection $\mathrm{LOD}_{t}=0.95 \mathrm{nmol}$ $\cdot \mathrm{s}^{1 / 2}$ was obtained from sample volumes around $1 \mu \mathrm{l}$. 
The sensitivity of this approach is compared with contemporary methods that utilize solenoid, micro-stripline, and micro-slot probe microcoils. It is demonstrated that planar microcoils are very very comparable to these designs in sensitivity, with the added advantage of being a flexible platform. 


\section{Acknowledgements}

I would like to thank my advisor, Professor Marcel Utz for his teaching, guidance, encouragement, and near saintly patience that allowed me the opportunity to pursue this challenging and unique research project. I would also like to thank Professors David Cafiso and Hossein Haj-Hariri for taking time out of their busy schedule to be on my committee. The contributions of Anja Zaß, Dr. Jan Korvink, Dr. Kailiang Wang, Jon Elliot, Kyle Lamson, and Dr. Reza Monazami to the early stages of this project are gratefully acknowledged. This work was supported by U.S. National Science Foundation under grant number CHE-0809795 and by the National Institute of Health under award number 5R01EB011591.

Lewis Steva, Joe Beatrice, Alex Lobo, and Ed Spencely were all immensely helpful in any number of tasks ranging from equipment repair to fabrication work. Thanks to my colleagues Dr. Gavin Garner, Brian Poe, Dr. Jenny Lounsbury, Dr. Carmen Reedy, Dr. Murali Ghatkesar, Dr. Suk-Heung Song, Dr. John Gaskins, Kerui Xu, Trevor Kemp, Dr. Francisco Lara, and Dr. Huihui Wang for thoughtfully replying to my endless emails and providing impromptu training sessions. The graduate community at large, most especially from the academical village, must also be acknowledged for their camaraderie in commiserating the woes upon the life of a graduate student.

Finally, thanks to my friends and family for support and encouragement every step of the way. 


\section{Contents}

$\begin{array}{ll}\text { Abstract } & \text { ii }\end{array}$

Acknowledgements

List of Figures viii

List of Tables $\quad$ xi

Abbreviations $\quad$ xii

Symbols $\quad$ xiii

1 Motivation 1

2 MicroNMR - Theory 5

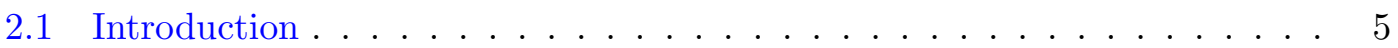

2.2 Fundamentals of NMR . . . . . . . . . . . . . . . . 7

2.2 .1 Spin Polarization . . . . . . . . . . . . . . . . 7

2.2 .2 Transverse Magnetization . . . . . . . . . . . . . . . . . . . . . 13

2.2 .3 Detecting a NMR Signal . . . . . . . . . . . . . . . . . . . . . . 17

2.2.4 Chemical Shift and Spin-Spin Coupling . . . . . . . . . . 18

2.3 Microfluidic NMR . . . . . . . . . . . . . . . . . . . . . . . . . . 21

2.3 .1 Sensitivity . . . . . . . . . . . . . . . . 23

2.3 .2 Resolution . . . . . . . . . . . . . . . . . . . . . . . . . . . 25

2.3 .3 Microfabricated Coils . . . . . . . . . . . . . . . 26

2.3.3.1 Solenoid . . . . . . . . . . . . . . . 27

2.3.3.2 Linear . . . . . . . . . . . . . . . . 28

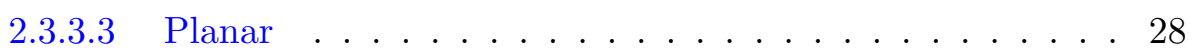

2.4 Conclusion . . . . . . . . . . . . . . . . . . . . . . 29

3 Integrated Planar Microcoils for Inductively Coupled NMR 30

3.1 Introduction . . . . . . . . . . . . . . . . . 30

3.1 .1 Prior Work . . . . . . . . . . . . . . . . 31 
3.1.1.1 Velocity Profile . . . . . . . . . . . . . . 32

3.1.1.2 Planar Microcoil Fabrication . . . . . . . . . . . 32

3.1 .2 Experiment Configuration . . . . . . . . . . . . . . . . 33

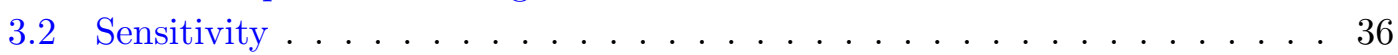

3.2.1 Limit of Detection . . . . . . . . . . . . . . . . . . . . . . . . . . . 37

3.2 .2 Planar Coils . . . . . . . . . . . . . . . . . . . . 37

3.2 .3 Cost of Inductive Coupling . . . . . . . . . . . . . . . . . 39

3.3 Resolution . . . . . . . . . . . . . . . . . . . . . . 41

3.4 Design . . . . . . . . . . . . . . . . . . . . . . . . . . . . . . . . . . . .

3.4 .1 Inductive Coupling . . . . . . . . . . . . . . . . . 44

3.4 .2 Microresonator Design . . . . . . . . . . . . . . . . 49

3.5 Fabrication Methods . . . . . . . . . . . . . . . . . . . 51

3.5.1 Fluidic Channels . . . . . . . . . . . . . . . . . . . 52

3.5.1.1 Glass Substrate . . . . . . . . . . . . . . . . . 52

3.5.1.2 Polymer Substrate . . . . . . . . . . . . . . . . . . . . . . . . . . 53

3.5 .2 Microcoil Lithography . . . . . . . . . . . . . . . . . 53

3.5.2.1 Glass Deposition . . . . . . . . . . . . . . . 54

3.5.2.2 Upisel-N Etching . . . . . . . . . . . . . . . . . . . . 55

3.6 Microcoil Characterization . . . . . . . . . . . . . . 56

3.6 .1 Network Analyzer . . . . . . . . . . . . . . . . . 56

3.6 .2 Microimaging . . . . . . . . . . . . . . . . . . . 58

3.7 NMR Experiments . . . . . . . . . . . . . . . . . . 58

4 Characterization and Performance of Optimized Planar Microcoils 61

4.1 Microcoil Characterization . . . . . . . . . . . . . . . . . . 61

4.1 .1 Resonance Frequency and Q Factor . . . . . . . . . . . . . . . . 62

4.1.2 Signal Strength Dependence on Applied Power . . . . . . . . . . . 64

4.2 Lineshape and Signal/Noise Ratio . . . . . . . . . . . . . . . . . . . . 67

4.3 Limit of Detection . . . . . . . . . . . . . . . . . . . 69

4.4 Experimental Results . . . . . . . . . . . . . . . . . . . 71

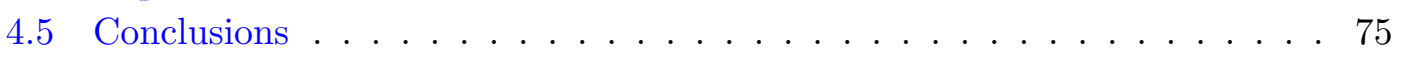

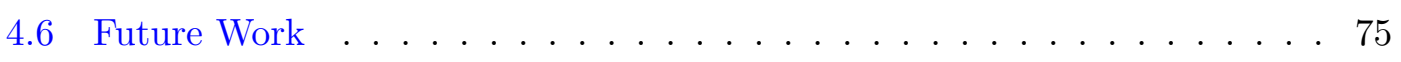

$\begin{array}{llr}5 & \text { Final Remarks } & 78\end{array}$

$\begin{array}{lr}\text { A Inductive Coupling Calculation } & 79\end{array}$

B Mathematica Coil Calculations $\quad 86$

$\begin{array}{lr}\text { C Microfabrication Masks } & 92\end{array}$

$\begin{array}{ll}\text { D Mathematica Network Analyzer Data Processing } & 97\end{array}$

E Mathematica FID and Imaging Data Processing 105 


\section{List of Figures}

1.1 Basic microfluidic NMR concept . . . . . . . . . . . . . . . 2

1.2 Microfluidic chip assembly . . . . . . . . . . . . . . 3

2.1 NMR experimental schematic . . . . . . . . . . . . . . . . . 6

2.2 Bar magnet and atom . . . . . . . . . . . . . . . 8

2.3 Ensemble of spins in applied field . . . . . . . . . . . . . . . . . . . 9

2.4 Spin up and spin down . . . . . . . . . . . . . . . 10

2.5 Magnetic dipole moment precession . . . . . . . . . . . . . . . . . 11

2.6 Net magnetization being flipped into the xy-plane . . . . . . . . . . . . . 14

2.7 Magnetization vector trajectory and decay . . . . . . . . . . . . . . 17

2.8 First recorded NMR signal . . . . . . . . . . . . . . . . . . . . . 18

2.9 Experimental ethanol spectrum with idealized J-couplings . . . . . . . . . . 19

2.10 Statistical spin distribution in ethanol . . . . . . . . . . . . . . . . 21

2.11 Metabolomics . . . . . . . . . . . . . . . . . . 22

2.12 Paramagnetism and diamagnetism . . . . . . . . . . . . . . . . 25

2.13 Lineshape prediction from material type . . . . . . . . . . . . . 26

2.14 Review of $\mu$-coil NMR designs . . . . . . . . . . . . . . . . 27

3.1 Velocity profile with hand wound solenoid . . . . . . . . . . . . . 32

3.2 Farbrication of planar microcoil LOC device . . . . . . . . . . . . . . . 33

3.3 Experimental setup schematic . . . . . . . . . . . . . . . . . . 34

3.4 NMR console overview . . . . . . . . . . . . . . . . . . . 35

3.5 SNR versus $\mathrm{Z} / r_{0}$ for solenoid and planar coils . . . . . . . . . . . . . . 39

3.6 Tank circuit and predicted sensitivity ratio . . . . . . . . . . . . . 40

3.7 Profile of coil holder . . . . . . . . . . . . . . . . . . . . . . . 42

3.8 Pickup coil designs . . . . . . . . . . . . . . . . . . . . 45

3.9 Inductive coupling circuit diagram . . . . . . . . . . . . . . . . . . . . 46

3.10 Experimental tuning curve . . . . . . . . . . . . . . . . . . . . . . . . . . . . . . . . . . . . . .

3.11 Planar coil and comb capacitor . . . . . . . . . . . . . . . . . . . . . . . . . . . . . . . . .

3.12 Redesign of self resonant microcoil . . . . . . . . . . . . . 51

3.13 Glass substrate microcoil fabrication . . . . . . . . . . . . . . . 54

3.14 Copper laminate substrate microcoil fabrication . . . . . . . . . . . . . . 55

3.15 Fabricated planar microcoil examples . . . . . . . . . . . . . . . 56

3.16 Sniffer coil and measure coil resonance . . . . . . . . . . . . . . 57

3.17 Cancelation device, circuit diagram, and typical cancellation curve . . . . 58 
3.18 MRI of coil intensity . . . . . . . . . . . . . . . . . . . 59

3.19 Probe assembly schematic . . . . . . . . . . . . . . . . 60

4.1 Resonance frequency and quality factor vs. coil turns . . . . . . . . . . . 62

4.2 Fabrication errors . . . . . . . . . . . . . . . . . . . . 64

4.3 Gradient echo images of microcoils . . . . . . . . . . . . . . . 65

4.4 Signal intensity vs. excitation . . . . . . . . . . . . . . . 6 6 . . . . . 66

4.5 Comparison of rectangular and circular fluidic chambers . . . . . . . . . 67

4.6 Signal and noise in the time domain . . . . . . . . . . . . . . 69

4.7 Nutation experiment . . . . . . . . . . . . . . . . . . . . 71

4.8 Glucose 1D spectra . . . . . . . . . . . . . . . . . . . . . 72

4.9 COSY 2D spectrum . . . . . . . . . . . . . . 73

4.10 Preliminary computational results for susceptibility mismatched materials 76

4.11 Next generation probe assembly schematic . . . . . . . . . . . . . . . 77

A.1 Inductive coupling calculation part (a) . . . . . . . . . . . . . . 80

A.2 Inductive coupling calculation part (b) . . . . . . . . . . . . 81

A.3 Inductive coupling calculation part (c) . . . . . . . . . . . . . . . 82

A.4 Inductive coupling calculation part $(\mathrm{d}) \ldots \ldots$. . . . . . . . . . 83

A.5 Inductive coupling calculation part (e) . . . . . . . . . . . . . . 84

A.6 Inductive coupling calculation part $(\mathrm{f}) \ldots \ldots \ldots \ldots$. . . . . . . . 85

B.1 Microcoil inductance calculations part (a) . . . . . . . . . . . . . 87

B.2 Microcoil inductance calculations part (b) . . . . . . . . . . . . . 88

B.3 Wheeler formula coil calculations part (c) . . . . . . . . . . . . . . . 89

B.4 Wheeler formula coil calculations part (b) . . . . . . . . . . . 90

B.5 Wheeler formula coil calculations part (c) . . . . . . . . . . . . . 91

C.1 Microfluidic chip mask . . . . . . . . . . . . . . . . . . . . 93

C.2 Microcoil mask $1 \ldots \ldots \ldots$. . . . . . . . . . . . . . 94

C.3 Microcoil mask $2 \ldots \ldots \ldots \ldots \ldots$. . . . . . . . . . . . . 95

C.4 Adjustable microcoil mask . . . . . . . . . . . . . . . . . . . 96

D.1 Microcoil data processing part (a) f . . . . . . . . . . . . . 98

D.2 Microcoil data processing part (b) . . . . . . . . . . . . . . . . . . . . . . . . . . . . 99

D.3 Microcoil data processing part (c) . . . . . . . . . . . . . . . . . 100

D.4 Microcoil data processing part (d) f . . . . . . . . . . . . . 101

D.5 Microcoil data processing part (e) f . . . . . . . . . . . . 102

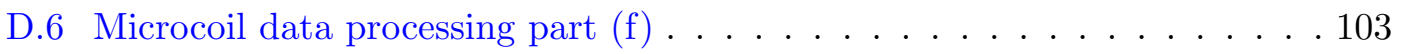

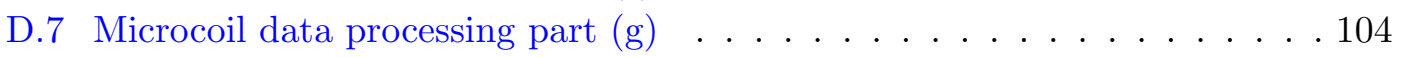

E.1 Varian data import routine part (a) . . . . . . . . . . . . . . 106

E.2 Varian data import routine part (b) . . . . . . . . . . . . . . . . . 107

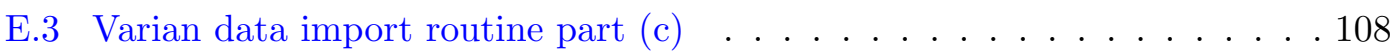

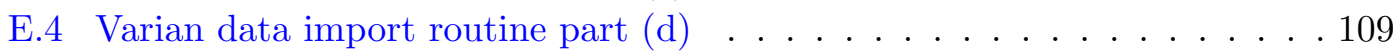

E.5 Mathematica spectra processing routine part (a) . . . . . . . . . 110 
E.6 Mathematica spectra processing routine part (b) . . . . . . . . . . . 111

E.7 Mathematica spectra processing routine part (c) . . . . . . . . . . . 112

E.8 Mathematica spectra processing routine part $(\mathrm{d}) \ldots \ldots .$. . . . . . . . 113

E.9 Mathematica spectra processing routine part (e) . . . . . . . . . . . . 114

E.10 Mathematica spectra processing routine part (f) . . . . . . . . . . . . . . 115

E.11 Mathematica spectra processing routine part $(\mathrm{g}) \ldots \ldots . . \ldots 116$

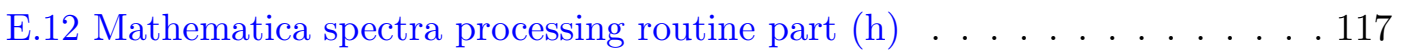

E.13 Mathematica image processing routine . . . . . . . . . . . . . 118 


\section{List of Tables}

2.1 Summary of non-Boltzmann techniques . . . . . . . . . . . . . . 24

3.1 Magnetic susceptibility list . . . . . . . . . . . . . . . . . 42

3.2 Coil design history . . . . . . . . . . . . . . . . . . . . . . . . . . . . . . . .

3.3 Substrate fabrication method comparison . . . . . . . . . . . 52 


\title{
Abbreviations
}

\author{
LOC Lab-on-a-Chip \\ NMR Nuclear Magnetic Resonance \\ MRI Magnetic Resonance Imaging \\ SNR Signal-to-Noise Ratio \\ LOD Limit Of Detection \\ RF Radiofrequency \\ DNP Dynamic Nuclear Polarization \\ CIDNP Chemically Induced Dynamic Nuclear Polarization \\ Q-factor Quality-factor \\ PMMA Poly(methylmethacrylate) \\ CAD Computer-Aided Design
}




\section{Symbols}

$\begin{array}{lll}B_{0} & \text { static field } & \mathrm{T} \\ T_{c} & \text { critical temperature } & \mathrm{K} \\ B_{1} \& B_{R F} & \text { radiofrequency pulse } & \mathrm{T} \\ \hat{\mu} & \text { magnetic dipole moment } & \\ \vec{L} & \text { angular momentum } & \\ I & \text { spin quantum number \& current } & \\ m & \text { integer } & \\ \gamma & \text { magnetogyric ratio } & \\ \omega_{0} & \text { Larmor frequency } & \mathrm{MHz} \\ \omega & \text { angular frequency } & \mathrm{MHz} \\ E & \text { energy } & \\ T_{1} & \text { spin-lattice relaxation time } & \mathrm{s} \\ t & \text { time \& thickness of comb capacitor finger } & \\ \mathbb{B} & \text { Boltzmann factor } & \\ { }^{1} \mathrm{H} & \text { hydrogen atom } & \mathrm{nV} \\ \hat{\Omega} & \text { rotating coordinates angular momentum } & \\ T_{2} & \text { transverse relaxation time } & \mathrm{s} \\ \hat{M} & \text { magnetization dipole } & \\ \xi & \text { Faraday voltage } & \\ \delta & \text { chemical shift \& skin depth } & \\ & & \\ & & \\ & & \\ & & \end{array}$


$J$

$\chi$

V

K

$R_{\text {coil }}$

$r$

$N_{S}$

$\Delta f$

$r 0 \& D i$

$r f \& r$

$\Delta$

$w$

$t$

$\rho$

$V_{\mu}$

$V_{T}$

$R_{\mu}$

$R_{T}$

$k$

$M_{\mu} \& M$

$L_{\mu}$

$L_{T}$

$C_{\mu}$

$C_{T}$

$Q_{\mu}$

$Q_{T}$

$\eta$

$f$

$N_{f}$

$d$

$x_{0}$ coupling constant

magnetic susceptibility

sample volume

$\mathrm{mm}^{3}$

sample concentration

$\mathrm{mMol}$

$\Omega$

$\mathrm{mm}$

$\mathrm{kHz}$

$\mathrm{mm}$

$\mathrm{mm}$

$\mathrm{mm}$

$\mathrm{mm}$

$\Omega$

$\Omega$

$\Omega$

pickup coil resistance

coupling constant

mutual inductance

microcoil inductance

pickup coil inductance

microcoil capacitance

pickup coil capacitance

microcoil Q-factor

pickup coil Q-factor

power efficiency

frequency

number of comb capacitor fingers

distance from finger to finger $\quad \mu \mathrm{m}$

overlap distance $\quad \mu \mathrm{m}$ 
$\Omega \quad$ dissipated fraction of power in resonator $P$

power 


\section{Chapter 1}

\section{Motivation}

Microfluidic Lab-on-a-chip devices represent a rapidly emerging technology based on the paradigm of integrating complex biological or biochemical assays on a compact, expendable platform [1]. Substantial progress has been demonstrated in recent years, in particular in the development of on-chip methods for the culture, manipulation sorting, and characterization of cells. Integration of such devices with high-resolution spectroscopy offers obvious advantages; traditionally though, NMR coils are designed with high degrees of cylindrical symmetry to optimize filed homogeneity, which is interrupted by the introduction of planar microfluidic device samples. NMR spectroscopy is one of the few tools available that allows metabolome quantification of biological fluids non-invasively, without the use of fluorescent labels, and without destroying the sample.

As discussed in more detail in the following, due to the limited sample volumes, microfluidic NMR spectroscopy benefits from the use of miniaturized receiver coils. Several designs, such as solenoids wound around capillaries [2-6], planar microcoils [7-10], micro-striplines [11-14], and micro-slot probes [15] have been described. However, these approaches require a fixed fluidic infrastructure to accommodate the sample. This runs 

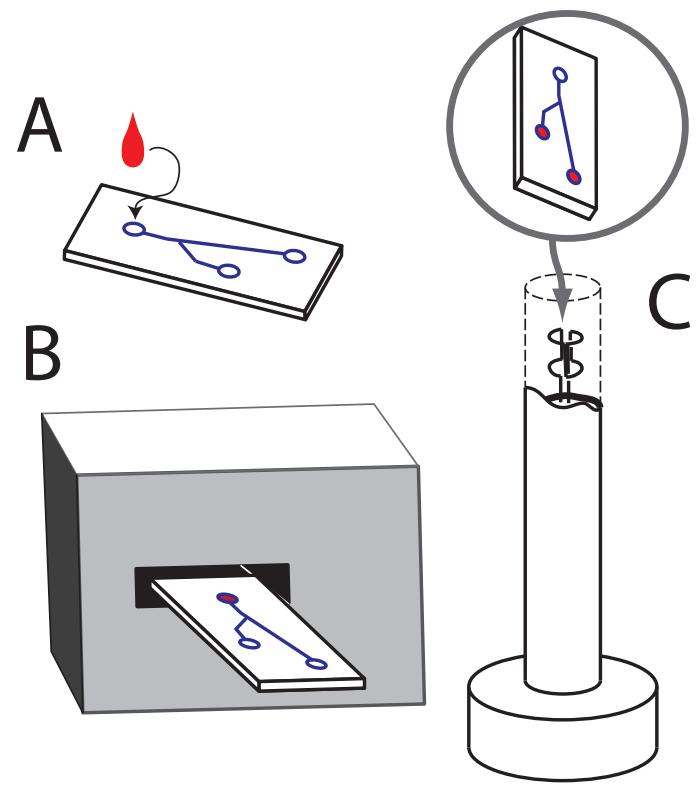

Figure 1.1: Basic concept of microfluidic NMR. A: Sample is loaded onto Lab-on-achip device; B: development box actuates microfluidic sample preparation/separation; C: chip is inserted into NMR probe assembly for spectroscopy.

counter to the paradigm of expendable LOC devices, and is difficult to combine with high-throughput clinical screening applications (Figure 1.1 shows the basic concept of how microfluidic NMR works). By contrast, our approach relies on planar radio frequency $(\mathrm{RF})$ resonators that are integrated into the microfluidic platform. These are essentially 2D metal structures designed to resonate at the Larmor frequency, which are coupled inductively to the NMR receiver and transmitter. This mode of operation allows for easy insertion and removal of the microfluidic device from the spectrometer. It is important to note that inductive coupling does not incur a significant cost in sensitivity $[16,17]$. In fact, we found the sensitivity of spiral-coil resonators to exceed results that have been reported for conventionally coupled spiral coils of similar geometry $[7,8]$.

As has been shown both theoretically [18-21] and experimentally [22-24], the specific sensitivity (per mole of sample) of NMR measurements increases dramatically as the 

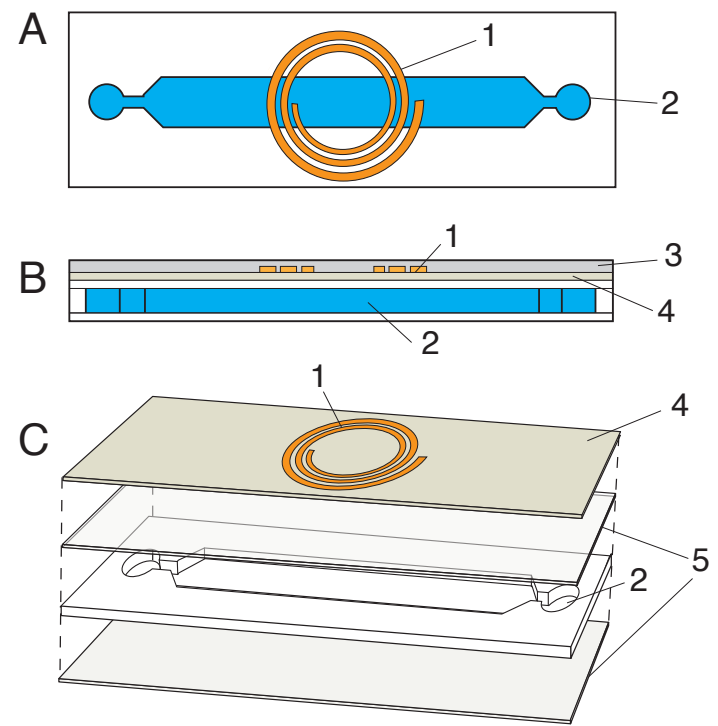

Figure 1.2: Integration of microfluidic chip with micro fabricated resonator. A: top view; B: cross-section view' $\mathrm{C}$ : assembly. a: $\mathrm{Cu} / \mathrm{Au}$ self-resonant planar micro coil; 2: fluidic network; 3: Protection dielectric (MEMS wax or SU-8); 4: coil substrate (polyimide/glass); 5: cover layers (PMMA/glass).

receiver coil is scaled down. This has been exploited extensively to devise "hyphenated" techniques [2, 3, 25-28] such as high performance liquid chromatography (HPLC)-NMR, in which the sample is injected after chromatographic separation into a capillary that runs through a miniaturized solenoid coil, or forms the dielectric in a micro-stripline. Microfluidic lab-on-a-chip devices, by contrast, have a planar geometry. Obtaining NMR signals with optimum sensitivity from such a device requires receiver coils with a similar form factor. Planar spiral coils can be made by lithographic techniques, and have been successfully integrated with simple microfluidic systems [7-10]. High sensitivity NMR signals can also be obtained from microfluidic systems by remote detection [4, 5, 29-32]. In this case, the signal is encoded by a large transmitter coil that fits around the entire microfluidic device. Detection, however, is done using a micro-solenoid wrapped around a capillary downstream from the microfluidic device. 
In all these cases, the integration of microfluidics with NMR spectroscopy requires either an electrical contact or a fluidic connection to the lab-on-a-chip device. This is inconvenient, and severely limits high-throughput applications.

Here, we present contactless microfluidic chips based on planar micro coils that can be coupled inductively to the NMR console, by simply inserting them into a probe assembly equipped with a probe coil that is large enough to accommodate the entire chip. The principle is illustrated in Figure 1.2. Inductive coupling is widely used in NMR imaging $[33,34]$. Recently, Sakellariou et al. have shown that substantial gains in sensitivity are possible in magic angle spinning experiments on mass-limited samples by including inductively coupled resonators into the MAS rotor [35]. As discussed in the remainder of this paper, planar spiral micro coils with the required self-resonance frequencies in the range of several hundred $\mathrm{MHz}$ and quality factors above 50 are easily obtained by simple lithographic techniques. Microfluidic devices can be integrated with NMR spectroscopy by simply attaching such resonators to their surface. Thus equipped, the microfluidic device is then just as easy to introduce and remove from the NMR probe as a conventional $5 \mathrm{~mm}$ sample tube. This offers considerable advantages, particularly in view of future clinical applications based on NMR metabolomics. 


\section{Chapter 2}

\section{MicroNMR - Theory}

\section{$2.1 \quad$ Introduction}

It was December of 1945 when two groups at Harvard and Stanford, respectively led by Edward Purcell and Felix Bloch, each independently detected weak radiofrequency signals from atomic nuclei (to be precise, the Harvard group was examining $1 \mathrm{~kg}$ of paraffin wax and the Stanford group was looking at water). Prior to this date nuclear spins had been detected by Isidor Rabi at Columbia, for which he received the 1944 Nobel Prize; but this was only accomplished using molecular particle beams, restricting the method to a small number of samples. It was a scientific revolution to realize that atomic nuclei could be observed in bulk for just the cost some RF signal processing electronics and a big magnet. For their contribution, Purcell and Bloch shared the 1952 Nobel Prize. Since that time the field of NMR spectroscopy has expanded as a interdisciplinary tool and is now used in many engineering disciplines, medicine, chemistry, biology, and physics. The primary reason for this large scale adoption of NMR is its ability to 
determine molecular structure of matter, intramolecular dynamics and intermolecular dynamics (most literature focuses on either liquids or solids with the occasional exception using gases [36]).

Atoms - composed of electrons, protons and neutrons - have some intrinsic physical properties. These are: mass, electric charge, spin, and magnetism (a consequence of charge and spin). Most important to NMR are the latter two, which interact with an applied magnetic field (see Figure 2.1). Although these are weak interactions they can be observed and manipulated with RF coils [37].

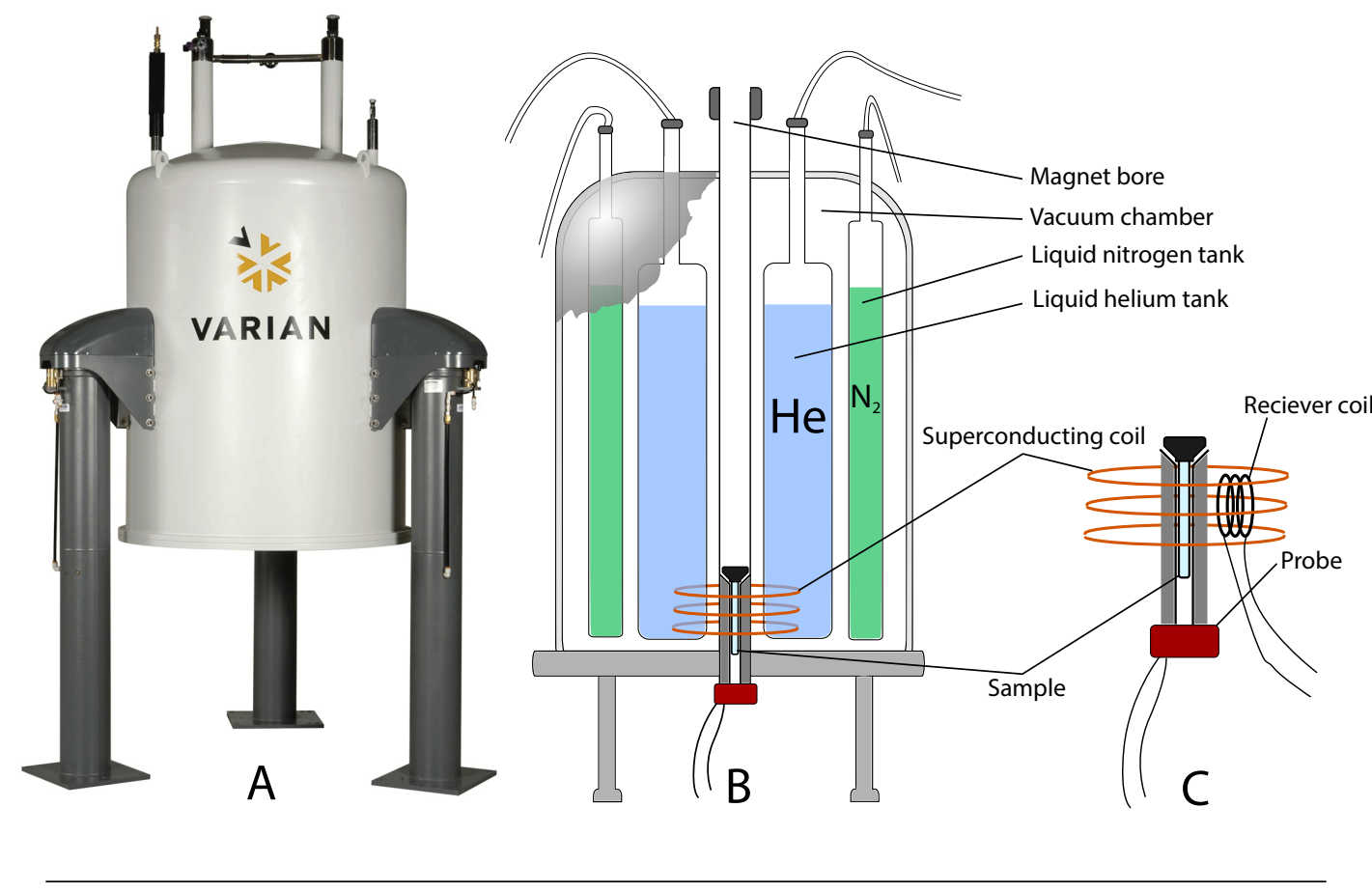

Figure 2.1: A: Typical exterior view of NMR spectrometer. B: Cutaway view of the superconducting magnet, which uses a helium to maintain the superconducting coil material temperature below $T_{c}$; a secondary layer of nitrogen surrounds the liquid helium shell to slow burn off of the much more expensive liquid helium. C: Enlarged view of receiver coil and probe geometry.

In modern NMR spectrometers a large static field $\left(\mathrm{B}_{0}\right)$ is generated by a superconducting magnet, which must be cooled below the critical temperature $\left(\mathrm{T}_{c}\right)$ of the superconducting 
material. Liquid helium is used for this purpose, which allows the magnetic core to maintain a temperature around $4 \mathrm{~K}$. Once a sample is immersed in this static field a RF-pulse $\left(B_{1}\right)$ is applied perpendicularly to the $B_{0}$ field to excite the nuclear spins out of their aligned ground state. The same RF-coil (now in receiver mode) is then used to record the ensuing changes in the magnetic field which induce small current fluctuations which are detected. This signal is then amplified, processed and delivered digitally to the user console where it can be Fourier transformed into a spectrum. As mentioned earlier, NMR spectroscopy is powerful in its ability to elucidate chemical structure. However, this information can only be interpreted if one possesses a strong understanding of the underlying physics.

This chapter will present a semi-classical view of NMR, and in particular look at its limitations in sensitivity relating to the present work focusing on microcoils and their application to microfluidic devices.

\subsection{Fundamentals of NMR}

\subsubsection{Spin Polarization}

Magnetic resonance is powerful in its ability to exploit the intrinsic magnetic dipole moment $(\hat{\mu})$ of a atom, which is idealized in Figure 2.2. The magnetic moment is a necessary consequence of angular momentum of a charged particle. For example, electrons possess electric charge (i.e. $1 e$ ) and as a result, when orbiting the nucleus, cause a magnetic dipole field (much like when running current through a coiled wire). 


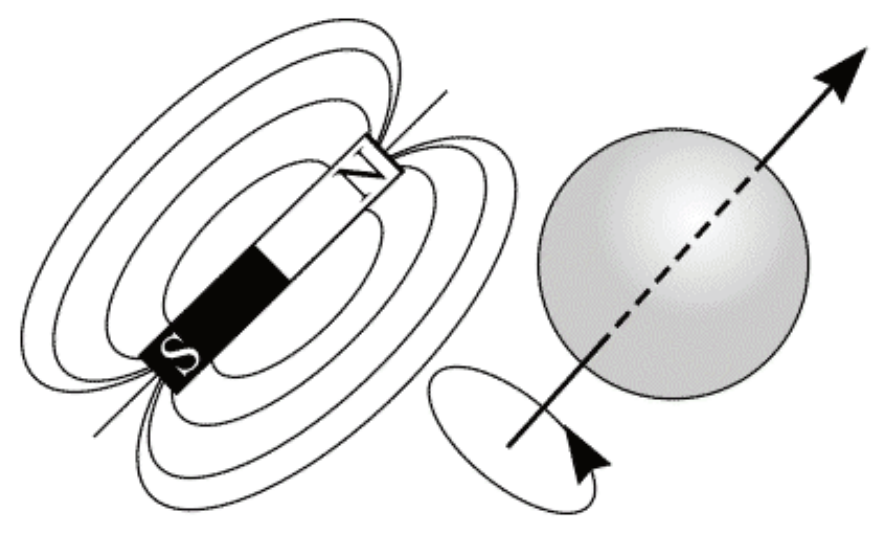

FiguRE 2.2: Juxtaposition of bar magnet and atomic magnetic moment which behaves like a miniature bar magnet [38]

Spin is also a form of angular momentum. However, it's not produced by rotation or movement of anything physical but is rather a intrinsic property of neutrons and protons. Due to the conservation of energy, stationary quantum states are eigenstates of the total angular momentum operator $L^{2}$ :

$$
\vec{L}^{2}=I(I+1) \cdot \hbar^{2}
$$

where $I$ is the spin quantum number with either a whole integer value for some particles $(0,1,2 \ldots)$ or a half integer value for others $(1 / 2,3 / 2,5 / 2 \ldots)^{1}$. The spin quantum number, $I$, is given by the nuclear ground state, and does not change within chemical processes. In the absence of a magnetic field, a spin I gives rise to $2 I+1$ degenerate levels, which can be characterized by their projection onto an arbitrary axis:

$$
L_{z}=m \cdot \hbar, \text { with } m=-I,-I+1, \cdots I
$$

A magnetic field $\left(B_{z}\right)$ lifts the degeneracy and splits the energy levels evenly as:

\footnotetext{
${ }^{1}$ Stern-Gerlach experiment first demonstrated half-integer spin by propelling silver atoms through a inhomogeneous magnetic field and observing the impact zones corresponding to spin up $(1 / 2 \hbar)$ and spin down $(-1 / 2 \hbar)[39]$.
} 


$$
E=\gamma \cdot B_{z} \cdot L_{z}=\gamma \cdot B_{z} \cdot m \cdot \hbar
$$

The magnetic dipole moment is related to the spin angular moment in the following equation:

$$
\overrightarrow{\hat{\mu}}=\vec{L} \cdot \gamma
$$

with $\gamma$ being the gyromagnetic ratio (or magnetogyric ratio) which varies in sign and value over a large degree depending upon the isotope in question. The orientation of the magnetic moment (for a paramagnetic material) is random in the absence of any applied magnetic field, so that over a large enough number of atoms the magnetic moments point equally in each direction ${ }^{2}$.

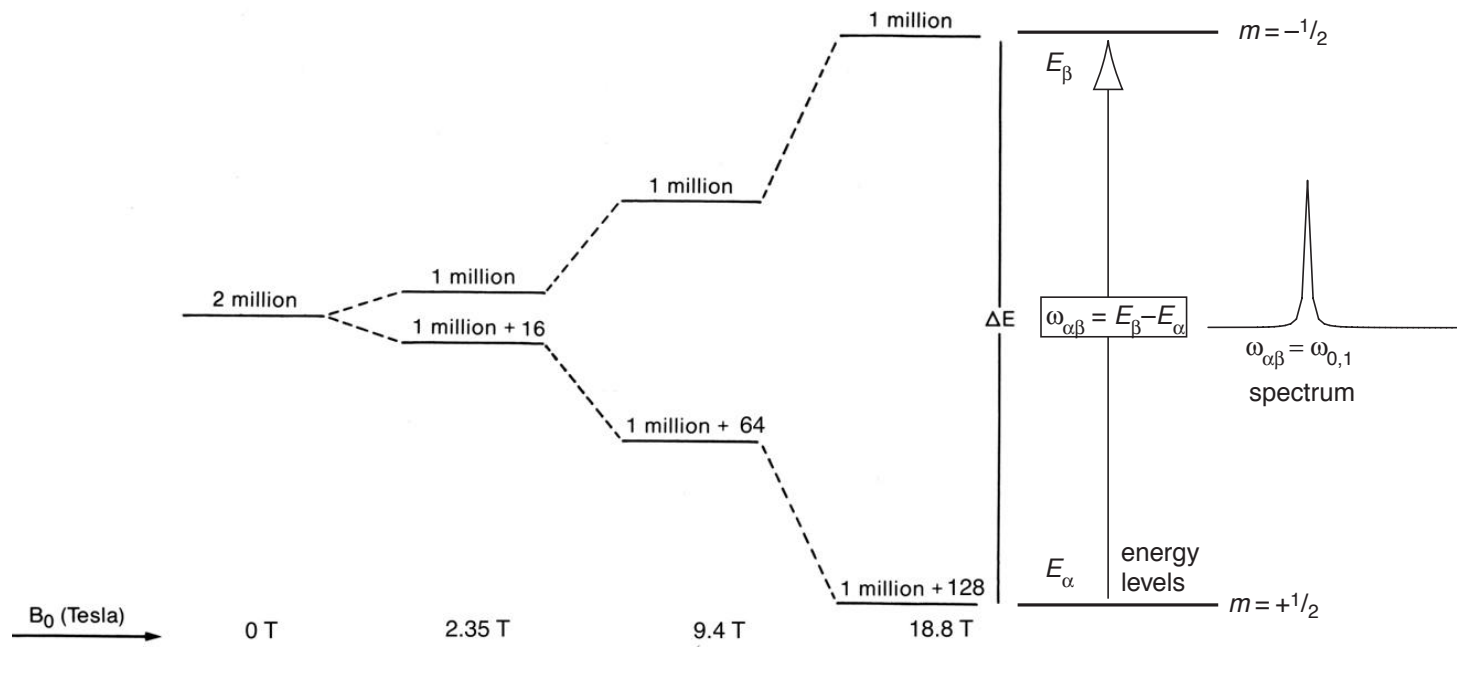

FiguRE 2.3: Left: Dependence of the separation of nuclear energy levels on magnetic field strength for $I=1 / 2$ and relative populations for two million protons in the sample [40]. Right: Transition between the two energy levels of a spin-half is allowed, and results in a single line at the Larmor frequency of the spin [41].

\footnotetext{
${ }^{2} \mathrm{~A}$ diamagnetic material has no dipoles in the absence of a magnetic field, whereas paramagnetic materials do but they average to zero in the bulk material.
} 
With the application of a magnetic field (see Figure 2.3), the spin system becomes quantized so that particles with spin $I$ will have $2 I+1$ sublevels (called the Zeeman effect). A second response causes the magnetic moments to start precessing around the direction of the external field ${ }^{3}$ (conventionally applied in the z-direction) called the spin polarization [37]. The angle $\theta$ that the cone (magnetic dipole moment precession) makes with the external magnetic field is dependent on the original direction of the magnetic moment and can have a value between $0^{0}$ and $180^{\circ}$. The rate of the precession is field dependent and is called the Larmor frequency $\omega_{0}$ :

$$
\omega_{0}=\gamma \cdot B_{0}
$$

Figure 2.4 shows that the the nuclei spin quantum number $I=1 / 2$, produces two possible orientations or energy levels that can be filled.
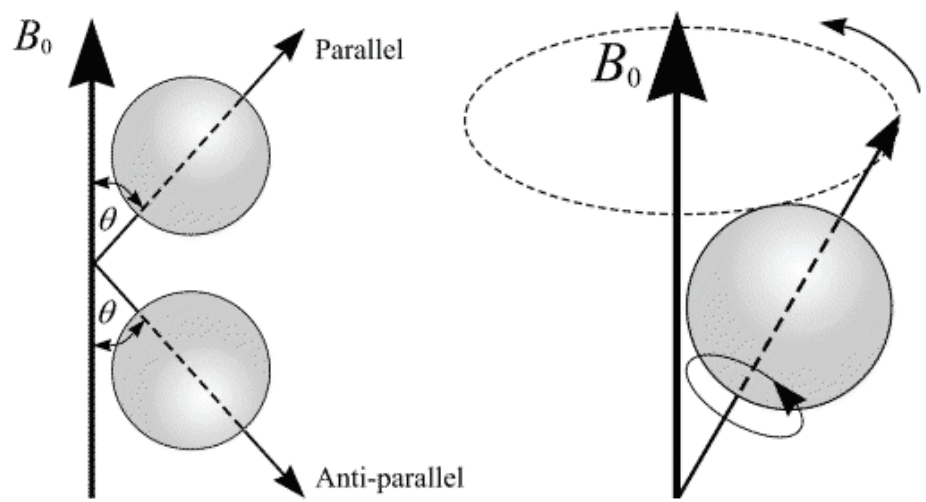

FiguRE 2.4: Left: In a external magnetic field the magnetic moments are constrained to adopted one of two orientations (i.e. spin up and spin down). Right: The magnetic dipole moment begin to precess in a cone shape around the applied magnetic field [38].

\footnotetext{
${ }^{3}$ From the Schrödinger equation, the spin precession can be described quantum mechanically as the harmonic time evolution of a superposition state, but for simplicity this explanation is omitted.
} 
These are known as spin-up and spin-down states and the energy difference between them can be written as:

$$
E=-\hat{\mu} \cdot B_{0},
$$

where the negative sign is interpreted as meaning that the lowest energy state for the spins is aligned with the $B_{0}$ field, or parallel, as opposed to anti-parallel (see Figure 2.5).

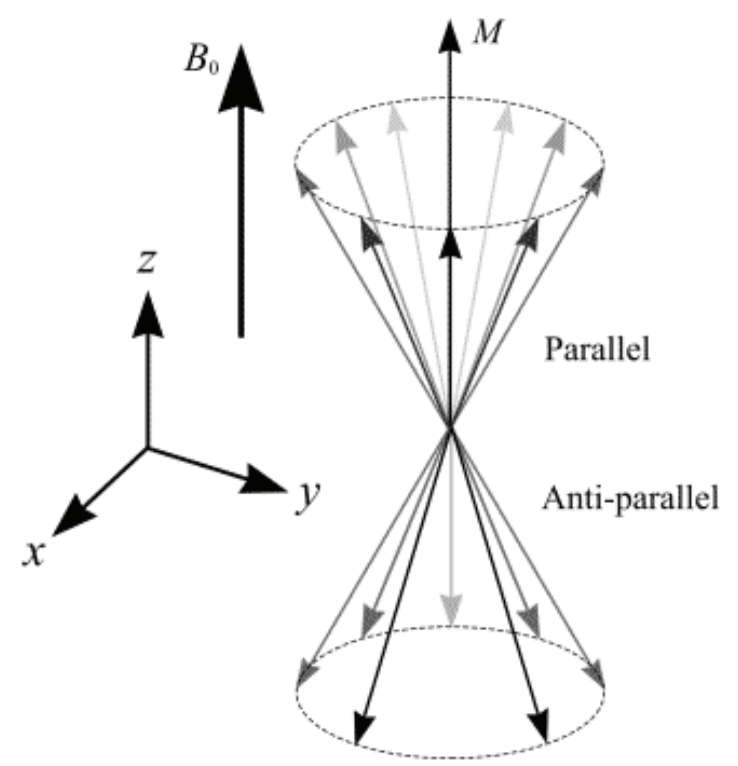

Figure 2.5: Ensemble of spins in the presence of a magnetic field produce a net magnetic dipole moment [38].

The interaction between atoms causes fluctuations around each nucleus so that equilibrium magnetization develops, which is the minimum thermodynamic potential, corresponding to the average sample magnetization in alignment with the magnetic field. The result can be seen in the net magnetization that starts to develop as a result of the preferred parallel direction of the magnetic dipole moments. The ramp time for the 
magnetization of the sample is dependent upon the spin-lattice/longitudinal relaxation time constant $\left(T_{1}\right)$ in the following formula:

$$
M_{z}(t)=\frac{\gamma^{2} \hbar^{2} N_{0} B_{0}}{3 k_{B} T}\left(1-2 e^{t / T_{1}}\right)=M_{0}\left(1-2 e^{t / T_{1}}\right),
$$

with $N_{0}$ being the number of spins and $M_{0}$ being the equilibrium magnetization, which can be related to the magnetic moment by $M_{0}=\frac{N_{0}}{\text { volume }} \overrightarrow{\hat{\mu}}$. The relaxation time $T_{1}$ depends upon the nucleus, temperature, viscosity and matter state. This value is typically in the range of ms to $\mathrm{s}$, but can be as long as days and years in extraordinary cases [37].

The primary reason NMR is such a inherently weak method of nuclei detection is because of very small populations of spin-states with different $I$ values. This means magnetization is often lost from offsetting spin populations. Illustrated in Figure 2.3 we see a difference of only 128 spins out of two million at a $18.8 \mathrm{~T}$ field strength. This is calculated from the Boltzmann factor, $\mathbb{B}$ for a room temperature system of ${ }^{1} \mathrm{H}$ particles. This expression measures the relative probability of spins being in one particular state in a multi state system, and can be written:

$$
\mathbb{B}=\frac{\hbar \gamma B_{0}}{k_{B} T}
$$

Figure 2.3 also indicates that in thermal equilibrium there is only a slight polarization of spin angular momentum vectors in the direction of the static external field, $B_{0}[37,42]$. 


\subsubsection{Transverse Magnetization}

To detect a RF signal from an ensemble of atoms, there must be some excitation from equilibrium (out of alignment with $B_{0}$ ) and an observation of the decay back to the lowest energy state (aligned with $\left.B_{0}\right)^{4}$. Practically this is accomplished by radiating the sample with a RF-pulse perpendicular to the static magnetic field, resulting in net magnetization being flipped into the xy-plane (see Figure 2.6), called transverse magnetization. This can be tracked using the right hand rule. For example, if the spin polarization starts in the $\mathrm{z}$-direction and is rotated by $\pi / 2$ about the $\mathrm{x}$-axis, the right hand rule can be applied to show that the resulting spin polarization is in the y-axis [37]. At the exact moment when the pulse stops, there would be a slight population difference between the spins polarized in the negative $y$-axis and those polarized in the positive y-axis, which is the source of the NMR signal. This is an oversimplification of transverse magnetization but a useful introduction. A more complete description is given heretofore.

The torque experienced by an ensemble of spins (represented as a dipole magnetization) by a external magnetic field can be expressed as:

$$
\frac{d \vec{L}}{d t}=\vec{M} \times \vec{B}
$$

where the external field is applying force on the spins to align in parallel, and $\mathrm{M}$ is the magnetization representing a dipole, which can be represented with the Curie Law:

$$
M_{z}=\frac{N \hbar^{2} \gamma^{2} B_{0} I(I+1)}{3 k_{B} T}
$$

\footnotetext{
${ }^{4}$ In the equilibrium longitudinal state, the spin magnetization is nearly undetectable [43].
} 

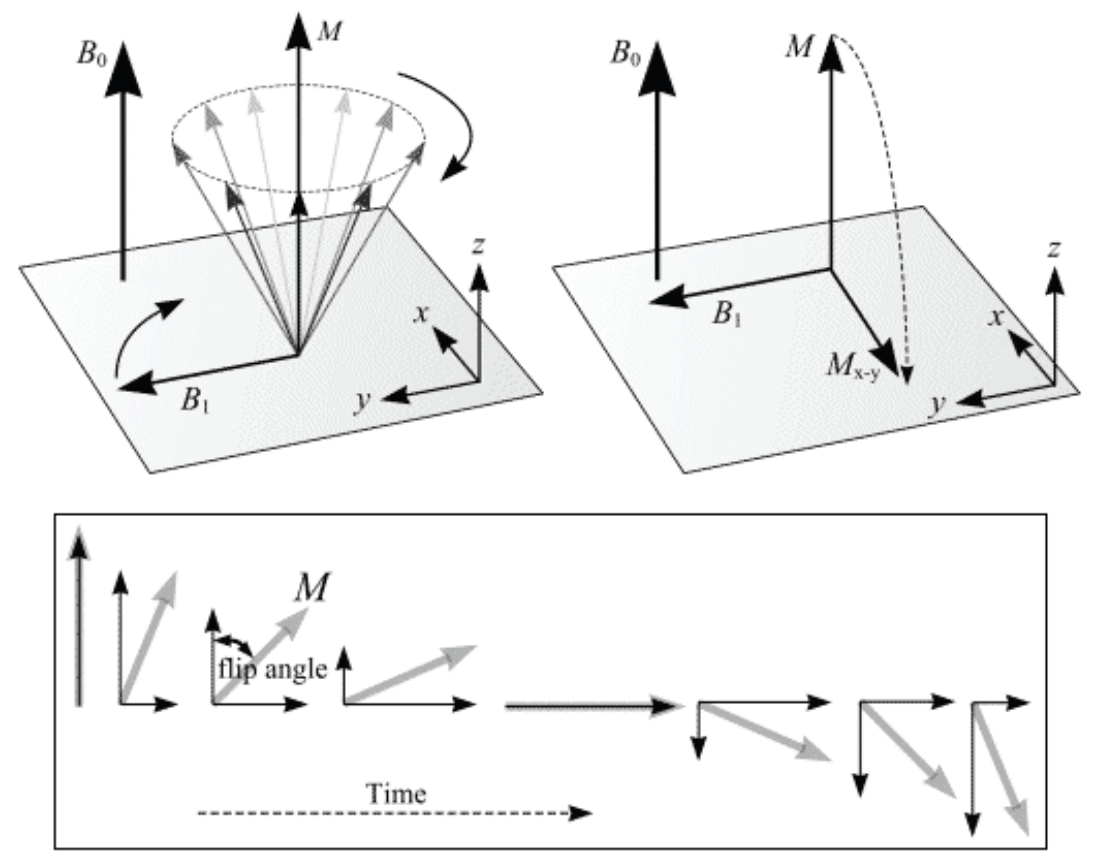

Figure 2.6: Top: Precession of the magnetic dipoles (shown left) creating net magnetization in alignment with the $B_{0}$ field. This magnetization is then flipped into the xy-plane (shown right). Bottom: Time progression of the flip angle between the z-axis and net magnetization as it is flipped into the xy-plane [38].

If Equation 2.4 and 2.9 are combined then the motion of the magnetic dipole moment in a external field can be described:

$$
\frac{d \vec{M}}{d t}=\gamma \vec{M} \times B_{0},
$$

As was described in the introduction to this section, to detect a RF signal the net magnetization must be flipped into the xy-plane by a second oscillating magnetic field, known as $B_{1}$ applied along either the x- or y-axis:

$$
B_{x}=2 B_{1} \cos (\omega t),
$$


where $B_{x}$ can be represented by two field vectors $B_{R}$ and $B_{L}$ of identical angular velocities, rotating in oppose directions [42]:

$$
\overrightarrow{B_{R}}=B_{1}(\vec{x} \cos (\omega t)+\vec{y} \sin (\omega t)) \text { and } \overrightarrow{B_{L}}=B_{1}(\vec{x} \cos (\omega t)-\vec{y} \sin (\omega t)),
$$

with $\vec{x}$ and $\vec{y}$ as respective unit vectors. The dynamics of applying $B_{1}$ in the presence of $B_{0}$ becomes complicated, and as such a rotating frame of reference is useful for elucidation. The rotating coordinates are thus given angular momentum $\vec{\Omega}$, which allows variations in the magnetic dipole, $\vec{M}$, to be expressed in terms of the unit vectors $\vec{i}, \vec{j}$, and $\vec{k}$ :

$$
\frac{d \vec{M}}{d t}=\frac{d M_{x}}{d t} \vec{i}+\frac{d M_{y}}{d t} \vec{j}+\frac{d M_{z}}{d t} \vec{k}+\frac{d \vec{i}}{d t} M_{x}+\frac{d \vec{j}}{d t} M_{y}+\frac{d \vec{k}}{d t} M_{z}=\frac{\partial \vec{M}}{\partial t}+\vec{M} \times \vec{\Omega}
$$

By setting Equation 2.11 equal to Equation 2.14 we derive the expression:

$$
\frac{\partial \vec{M}}{\partial t}=\gamma \vec{M} \times\left(B_{0}-\frac{\vec{\Omega}}{\gamma}\right)
$$

From this equation it's clear that there are two contributions to two resulting magnetic field in the rotating frame: one being static $\left(B_{0}\right)$ and the other being reduced, $\Delta B=$ $\vec{\Omega} / \gamma$. If the alternating field $B_{x}$, represented by $B_{R}$ and $B_{L}$ in the rotating frame, is now incorporated into Equation 2.15, the static contribution to the RF field will be represented by a RF field of magnitude $B_{1}$. For $\vec{\Omega}=\omega_{0}, \vec{M}$ will experience zero field. In the rotating frame of reference, the $\vec{M}$ will only rotate about the static time-independent part of the RF field, with a frequency defined by: 


$$
\omega_{1}=\gamma B_{1}
$$

To accomplish a flip of the net magnetization, so that it ends up parallel with the y-axis, the length of the RF pulse must follow:

$$
\tau_{90}=\frac{1}{4} \frac{2 \pi}{\gamma B_{1}} \sec
$$

In the laboratory reference frame, the net magnetization precesses at the Larmor frequency and induces current in the NMR detector coil. For some time interval, $t$, after the pulse, the magnetization in the $\mathrm{x}$ and $\mathrm{y}$ direction will have the form:

$$
M_{x}=M_{0} \sin \left(\omega_{0} t\right) e^{-t / T_{2}} \text { and } M_{y}=\cos \left(\omega_{0} t\right) e^{-t / T_{2}},
$$

where $T_{2}$ is the transverse relaxation time constant. $T_{2}$ is typically on the order of $T_{1}$ for small molecules in liquids. It characterizes the desynchronization of the magnetic dipole moments on a microscopic scale as they interact with their neighbors. The decay precesses at the Larmor frequency defined in equation 2.5. The movement described in equation 2.18 is visualized in Figure 2.7.

For a typical helix receiver coil (several centimeters in diameter) the induced voltage, or Faraday voltage, from the precessing nuclear magnetic moments, ignoring any tiny radiative contribution, can be written:

$$
\xi_{0}=\omega B_{1}\left(M_{x}+i M_{y}\right),
$$




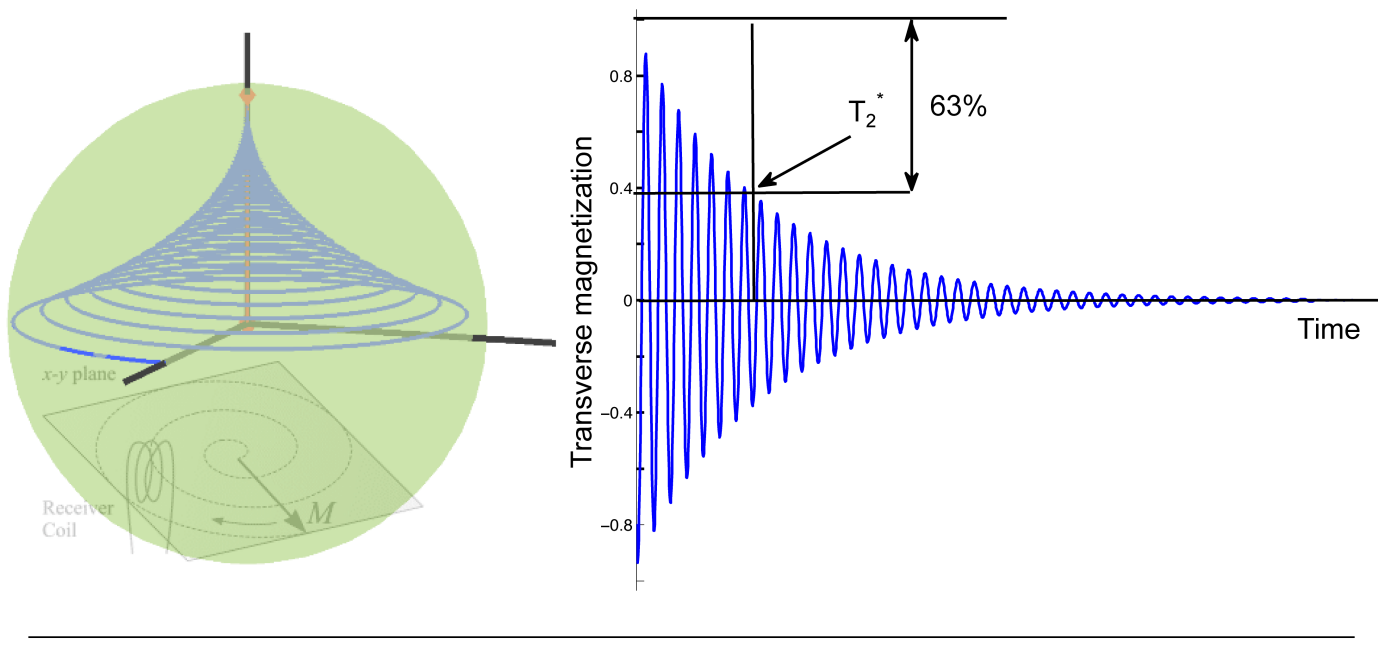

Figure 2.7: Left: Magnetization vector $(\vec{M})$ tip trajectory following the application of $90^{\circ} \mathrm{RF}$-pulse, assuming $\mathrm{T}_{1}=\mathrm{T}_{2}$. Right: Transverse magnetization decay and oscillation, called free induction decay (FID) $[38,44]$.

Values are typically on the order of several hundred nanovolts $(\mathrm{nV})$ before amplification.

Interfering noise is typically around 1-5 $\mathrm{nV}$, two orders of magnitude less than the signal $[45]$.

\subsubsection{Detecting a NMR Signal}

The oscillating transverse magnetization provides the opportunity to observe the nuclei, despite the fact that it is still relatively weak. For this purpose, a receiver coil is situated close the sample so that the changing magnetic field from the precessing nuclear magnetization can induce changes in the electric field to set electrons in motion and produce a detectable current in the receiver coil ${ }^{5}$. The macroscopic magnetic induction flux density generated by a rotating magnetization is given by:

$$
B_{1}(t)=\mu_{0} M(t),
$$

\footnotetext{
${ }^{5}$ In early NMR spectrometer designs, separate transmitter and reliever coils were used. Today only a single transceiver coil is employed for this purpose which performs both tasks.
} 
where $\mu_{0}$ is the vacuum permeability. Indicating that the signal does not go on indefinitely but decays quickly according to previously derived Equation 2.18.

After Fourier transforming the time domain free induction decay, we can visualize frequencies contained within the sample in the form of a spectrum, shown in Figure 2.8. This in turn provides detailed information on local environments within a nucleus and can be instrumental to understand the makeup of the molecule being studied. The amplitude of the respective peaks, gives the relative abundance of the spin states in the sample.

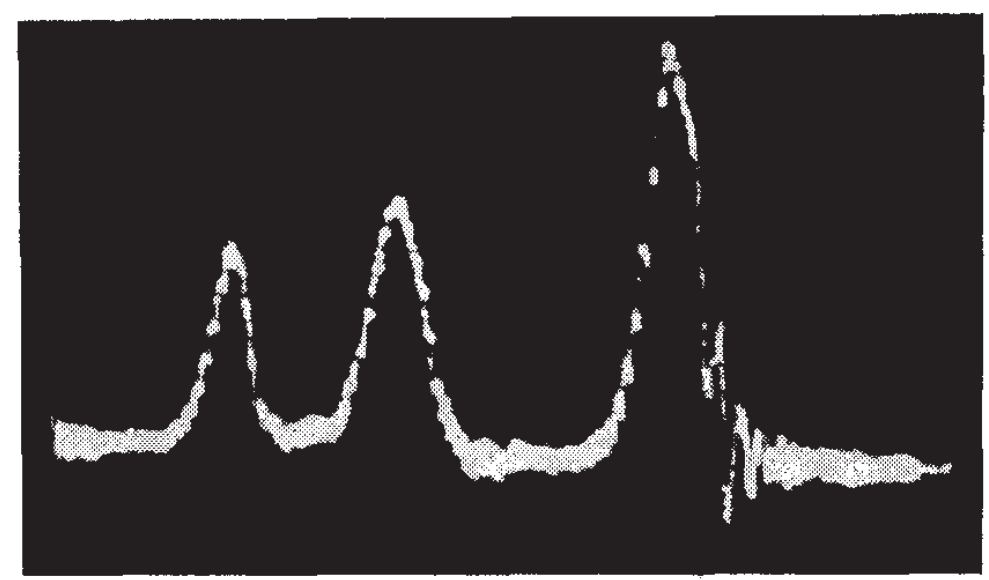

Figure 2.8: First ever recorded wide-line ${ }^{1} \mathrm{H}$ NMR spectrum from ethanol. The peaks from left to right are groups $-\mathrm{OH},-\mathrm{CH}_{2},-\mathrm{CH}_{3}[46]$.

\subsubsection{Chemical Shift and Spin-Spin Coupling}

To this point we have ignored that nuclei are surrounded by electrons which can be interacted with to distort the local magnetic field [37, 47]. An example is ethanol, shown in Figure 2.9, which has a $-\mathrm{CH}_{3},-\mathrm{CH}_{2}$, and $-\mathrm{OH}$ groups. The $-\mathrm{CH}_{3}$ and $-\mathrm{CH}_{2}$ groups see a slightly different magnetic field because of their local environment and 
correspondingly show up at different points along the spectrum. This phenomenon is known as chemical shift $(\delta)$ and stems from the magnetic coupling of electrons to the nucleus due to the motion of electrical charges. A second observation of the spectrum is the splitting of lines which is known as spin - spin coupling and comes from magnetic moments of the electron spin again coupling to the nucleus. In Figures 2.8 and 2.9 chemical shift allows us to identify three distinct groups. However, Figure 2.8 has such broad spectral lines that we are unable to identify the splitting that results from spin-spin coupling.

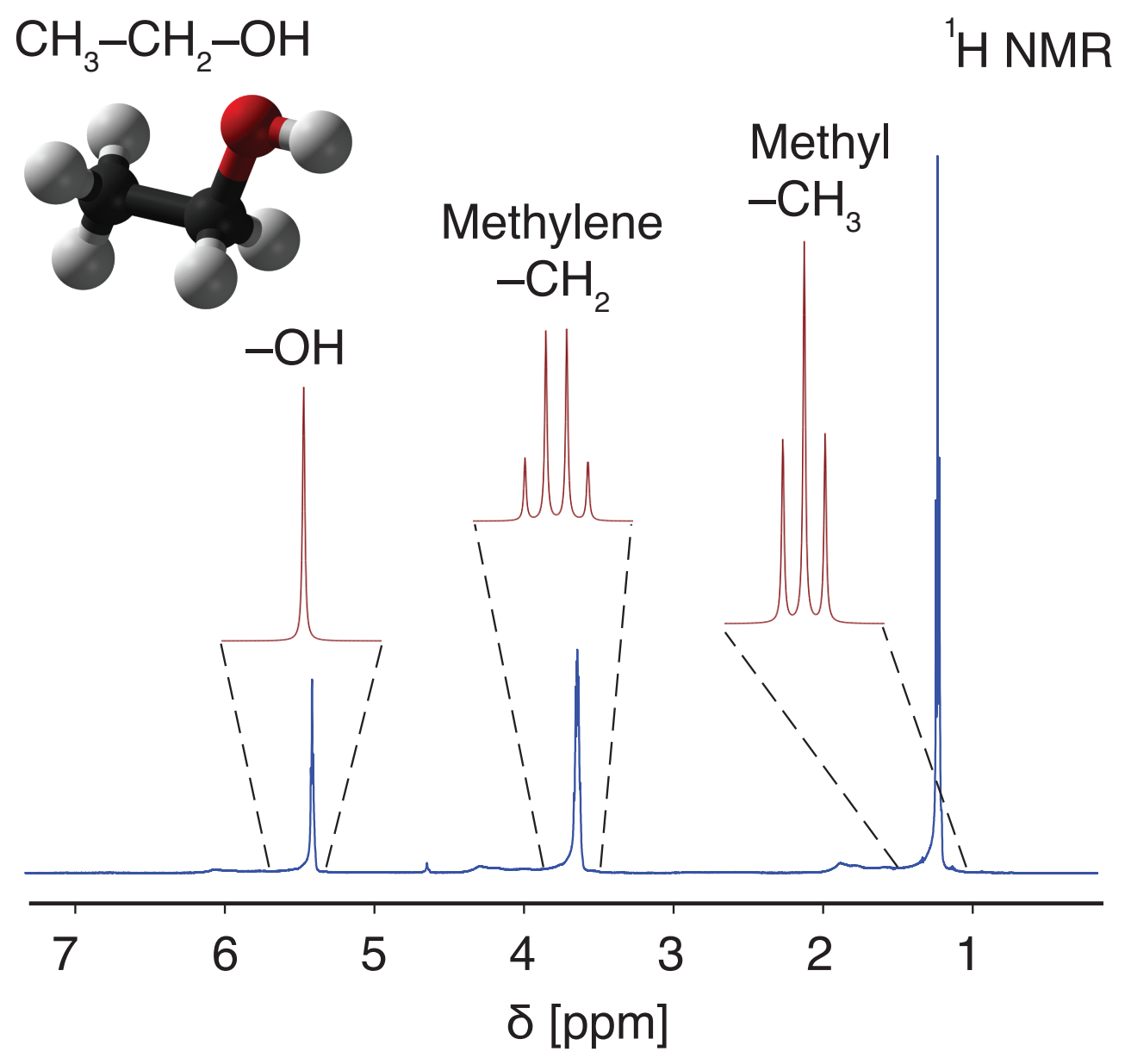

Figure 2.9: ${ }^{1} \mathrm{H}$ NMR experimental ethanol spectrum obtained from a microfuidic device, displayed in blue with the corresponding enlarged idealized lineshape shown in red for each nuclei. The peaks from left to right are groups $-\mathrm{OH},-\mathrm{CH}_{2}$, and- $\mathrm{CH}_{3}$. 
The most common form of NMR is ${ }^{1} \mathrm{H}$ NMR (otherwise known as proton NMR). Despite narrower chemical shifts than other available nuclei, the linewidths of this method are comparably sharp (which is particularly important when looking at spin-spin coupling). In Figure 2.9 we can interpret that the $-\mathrm{CH}_{3}$ or methyl group has a higher electron density and therefore has a lower chemical shift (i.e. the proton nuclei in the methyl group are more shielded than the $-\mathrm{CH}_{2}$ or methylene group). The size of the chemical shift also increases proportionally to the field size. The field-independent equation for the chemical shift can be written as:

$$
\delta=\frac{\omega_{0}-\omega_{0}^{T M S}}{\omega_{0}^{T M S}},
$$

where $\omega_{0}^{T M S}$ is the Larmor frequency of the reference compound (typically tetramethylsilane (TMS)).

Spin-spin coupling line splitting provides information on the number of chemically bonded nuclei around the studied nucleus. In Figure 2.10 this is shown using a ${ }^{13} \mathrm{C}$ NMR spectrum of methylene. The spacing between the lines is known as the coupling constant $(J)$. The peak intensities are 1:3:3:1 corresponding to the statistical spin distribution in the molecule. The integrated area below the peaks tells the number of nuclei that caused the line.

The combination of spin-spin coupling and chemical shift give insights on the nuclear and electronic environments of observed nuclei. 


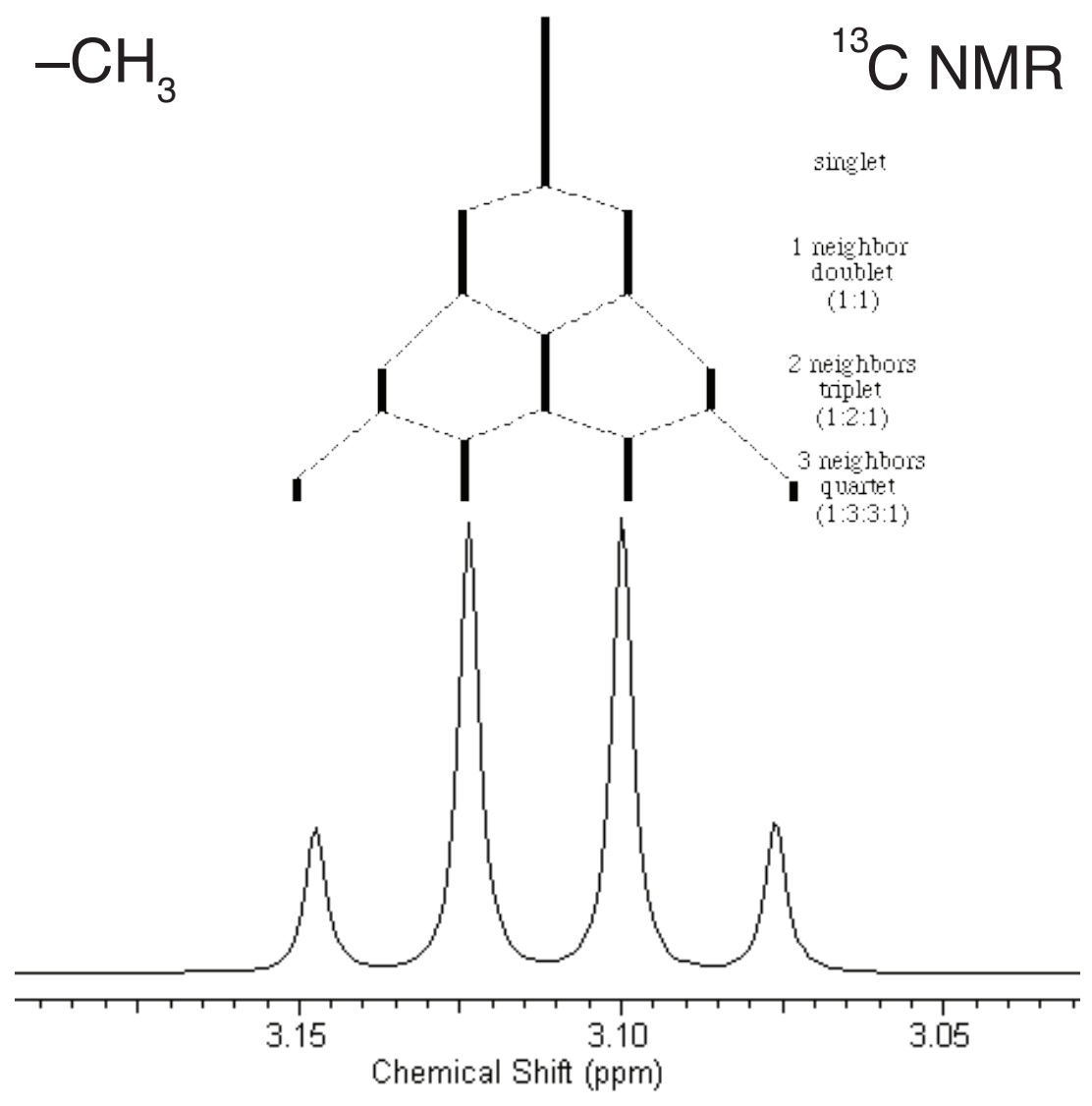

Figure 2.10: ${ }^{13} \mathrm{C}$ NMR methylene spectrum $\left(-\mathrm{CH}_{3}\right)[48]$.

\subsection{Microfluidic NMR}

Focus on mass and volume limited samples, as encountered in the field of microfluidic metabolomics, has pushed demand for highly sensitive non-invasive analytical instruments. NMR could be a indispensable tool in this field due to its capacity to produce unmatched chemical structural information along with interpolate intermolecular and intramolecular dynamics [49]. A major drawback is experimental insensitivity at small numbers of sample spins. The solution this thesis presents is based in the Bohr Correspondence Principle, relating the sensitivity of a NMR experiment to the probe 
efficiency $^{6}[50]$.

A second key concern of instrumentation work is resolution. For NMR applications to small volume metabolomics, this becomes problematic as samples typically contain hundreds to thousands to lines as seen in Figure 2.11, where each line corresponds to . Line broadening can be very detrimental in this case as entire signals can be erased (see Figure 2.8).

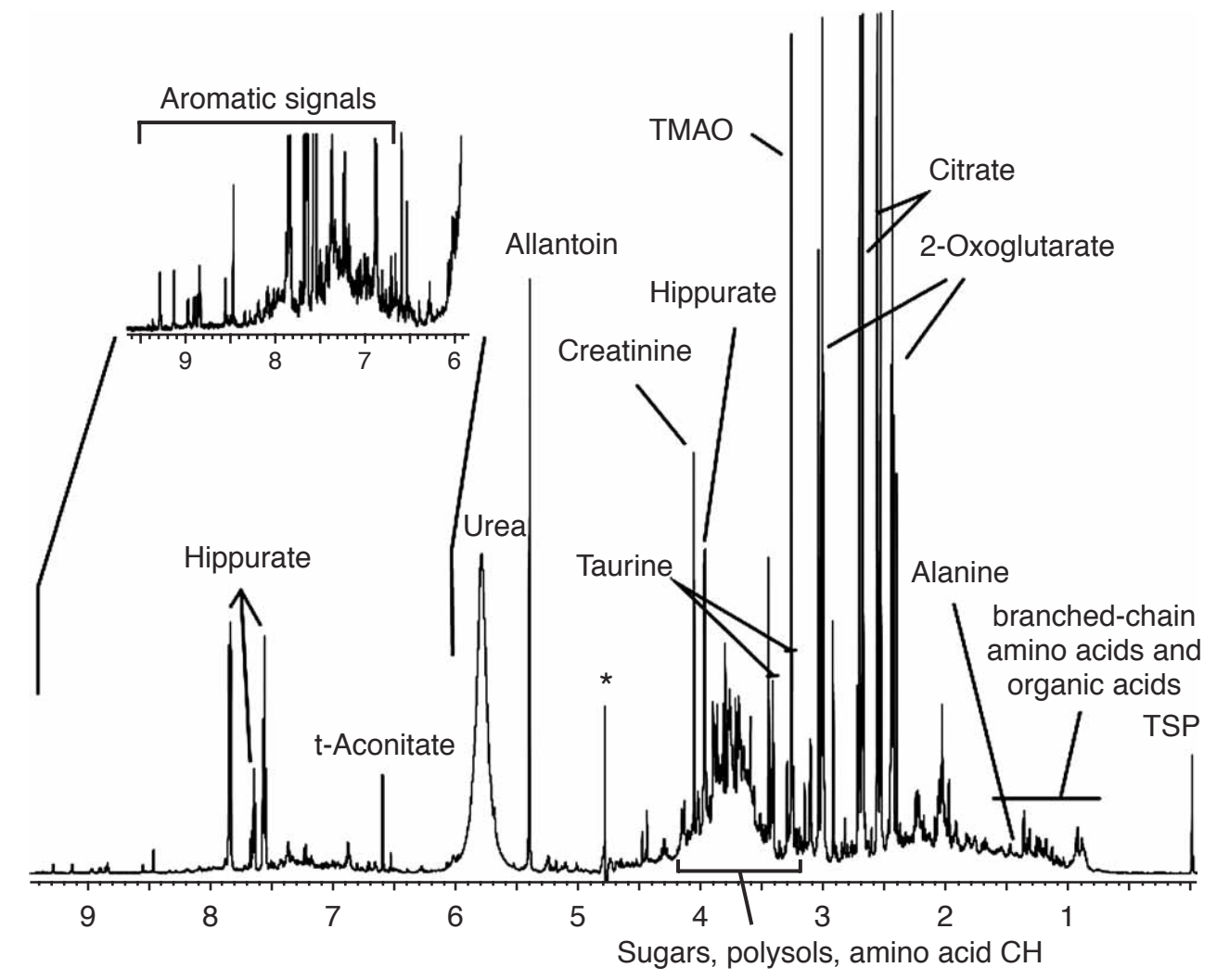

FigURE 2.11: $600 \mathrm{MHz}{ }^{1} \mathrm{H}$ NMR spectrum of control rat urine, displaying hundreds of resolved peaks. All peaks are referenced to the resonance to 3-trimethylsilylpropionic acid (TSP) at $0 \mathrm{ppm}$. The spectrum displays a wide range of metabolites such as aromatic, aliphatic compounds, sugars, amino acids and other osmolytes. The asterisk denotes the suppressed signal of water. TMAO, trim ethylamine N-oxide [51].

\footnotetext{
${ }^{6}$ Bohr's Correspondence Principle relates quantum mechanical behavior (microscopic) to classical physics (macroscopic)
} 
Blood plasma metabolites of interest have a concentration of tens of $\mu \mathrm{mol} / \mathrm{L}$ to $1-2$ mmol/L, meaning a suitable instrument should achieve a single scan SNR of 1000/ $\mu \mathrm{mol}$ for less than $2 \mu \mathrm{L}$ of sample and achieve a resolution linewidth approaching $2 \mathrm{~Hz}$. The following section is devoted to exploring the underlying limits of NMR sensitivity and resolution. Solutions will be presented that work to overcome these obstacles.

\subsubsection{Sensitivity}

The biggest hurdle preventing NMR to characterize small biological samples is sensitivity. Mass spectrometry, a competing metabolomic characterization method, has picomolar detection ability but is of course destructive to the sample and cannot detect molecular dynamics $[52,53]$. Additionally, mass spectrometry requires volatilization of the sample, which is not always quantitative, and can suffer from peak overlap. Optical techniques such as fluorescence and Raman spectroscopy can attain higher sensitivities than NMR but at the cost of sacrificing generality and/or specificity [54]. Fluorescence requires a specifically binding fluorophore which only detects a single metabolic species, making it unsuitable for some applications.

The mechanism driving NMR sensitivity is the number of available excitable spins in a sample. Within the field of NMR there exist methods to create non-Boltzmann populations which give several orders of magnitude increased sensitivity. These approaches are summarized in Table 2.1. All of these techniques rely on transfer of polarization of a medium (e.g. parahydrogen or the electron spin) to the nuclei of interest [55]; This approach only works in special cases. 
TABle 2.1: Non-Boltzmann Techniques Summary

\begin{tabular}{|l|l|l|}
\hline Technique & Advantages & Disadvantages \\
\hline DNP & $\begin{array}{l}\text { Dynamic nuclear polarization } \\
\text { (DNP) transfers polarization } \\
\text { from unpaired electrons for } \\
\text { signal enhancement of greater } \\
\text { than 10000 times [56, 57]. }\end{array}$ & $\begin{array}{l}\text { Polarization effect is less ef- } \\
\text { ficient at higher fields. Only } \\
\text { adapted for certain materials. } \\
\text { Requires two magnets. }\end{array}$ \\
\hline CIDNP & $\begin{array}{l}\text { Chemically induced dynamic } \\
\text { nuclear polarization (CIDNP) } \\
\text { is more amenable to liquid } \\
\text { state NMR [58]. }\end{array}$ & $\begin{array}{l}\text { Only adapted for certain solu- } \\
\text { tions. }\end{array}$ \\
\hline Para-Hydrogen & $\begin{array}{l}\text { Symmetry of para-hydrogen } \\
\text { is broken to produce highly } \\
\text { spin-polarized reactions [59]. }\end{array}$ & $\begin{array}{l}\text { Only adapted for certain solu- } \\
\text { tions. }\end{array}$ \\
\hline
\end{tabular}

While sensitivity gains of greater than 10000 times are extremely attractive, it is the lack of generality that limits these methods: only certain metabolites can be hyperpolarized, and the process is not quantitative. This can be a prohibitive use of the technology in cases of metabolomic high-throughput.

Even with hyperpolarization there is still a need for customized pickup coils to maximize sensitivity; but without hyperpolzarization there is still a expected sensitivity gain from optimizing existing RF-coils so that they tightly engulf the sample. This has the added advantage of reducing the Johnson noise ${ }^{7}$. Richard and Hoult determined in 1976 the sensitivity of an NMR coil to be inversely proportional to its diameter [19]. While gain factors are only 10-100 times sensitivity, these are sufficient to make experiments with nanoliter sample volumes practical $[22,23,60]$. Including a coupled resonator on the surface of the mircofluidic device, as described in the introduction, allows for a more flexible platform specifically designed for automated high-throughput applications.

\footnotetext{
${ }^{7}$ Johnson noise arises from the motion of the instruments electrons, in this case the RF-coil, at room temperature causing a random background signal [37].
} 


\subsubsection{Resolution}

Resolution in NMR spectroscopy refers to either (i) spectral resolution defined as the difference between spin populations that produce separable spectrum peaks[61-63], or (ii) line width. Each resonance line has an inherent width, depending on the resonance lifetime, and the homogeneity of the magnetic field.

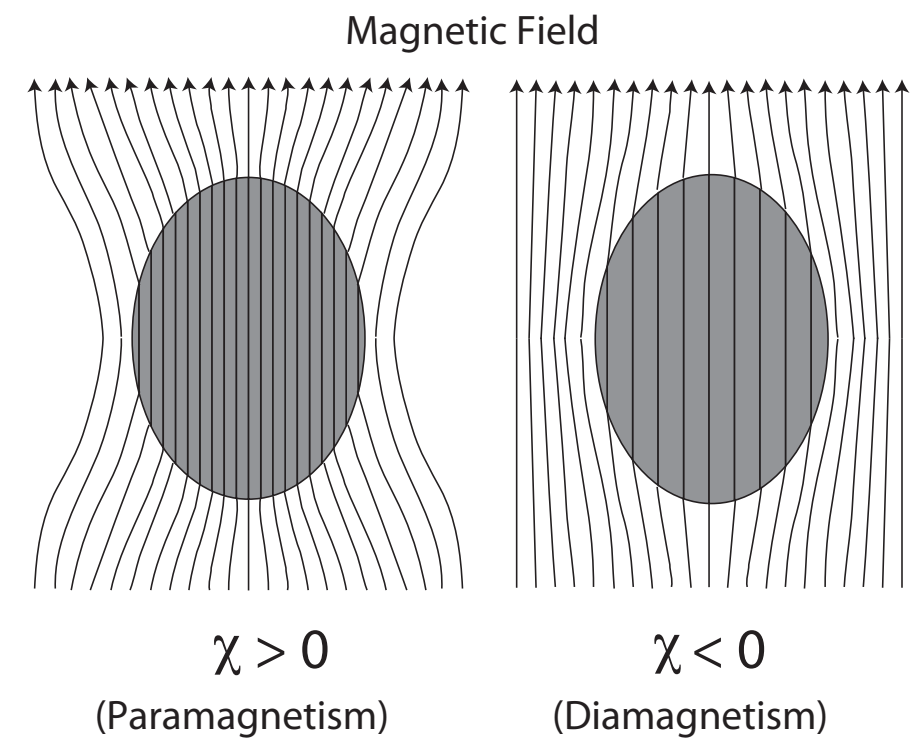

FiguRE 2.12: Applied external field shown distorted according to the magnetic susceptibility of the object [37].

High sensitivity NMR spectroscopy for microfluidics demands a large filling factor. Filling factor is percentage of a magnetic field occupied by sample. This means that the sample must be very close to the RF-coil for the best case filling factor ${ }^{8}$. Line broadening is a consequence of this close proximity and stems from magnetic susceptibility ${ }^{9}$ $(\chi)$ differences in the coil material, coil support system, sample holder, sample, and air present in the probe head. Jumps in susceptibility between materials cause small

\footnotetext{
${ }^{8} \mathrm{~A}$ wire wrapped around a capillary has a filling factor of 0.5 , which means half the magnetic field is occupied by sample

${ }^{9}$ Magnetic susceptibility indicates the sign and degree of magnetization when in the presence of a external field.
} 
variations in the homogeneity of the field which manifest as line broadening. Magnetic susceptibility of materials in a external field is shown in Figure 2.12. While this is not the focus of this thesis, it is still a motivating factor behind material selection for probe assembly and microcoil fabrication (see Figure 2.13).

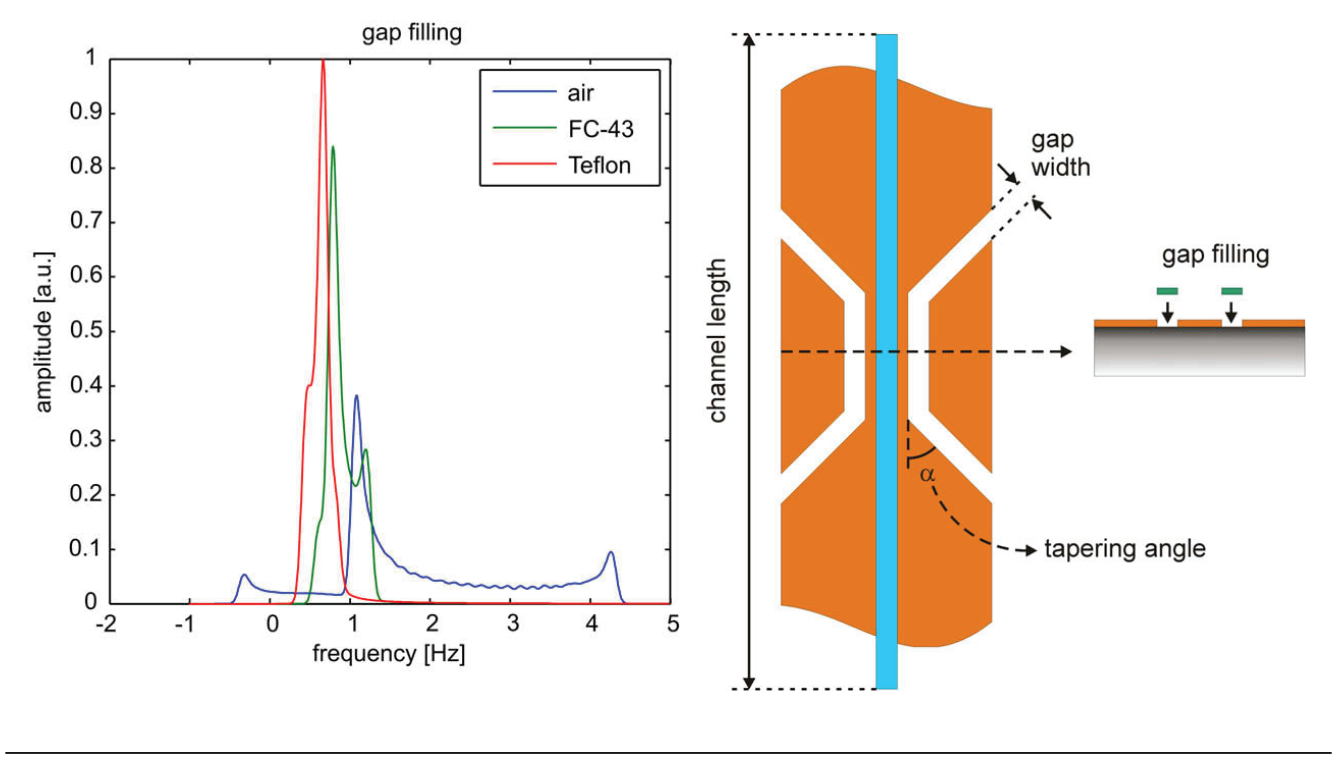

Figure 2.13: Lineshapes are predicted (left) for different gap type materials in the stripline microcoil configuration (right) [11].

\subsubsection{Microfabricated Coils}

While RF-coil miniaturization appears simple, the implementation presents challenges to preserve sample geometry, achieve highest possible sensitivity and resolution, and preserve compatibility with the entire catalog of NMR pulse experiments. The choice to inductively couple the microcoil to a larger pickup coil producing the $B_{1}$ field (i) eliminates the need to create electrical contacts to the chip and (ii) opens the possibility to investigate multiple samples on the same chip simultaneously. Thanks to the work of Webb et al. [22, 23] there has been substantial attention given to RF microcoils for 
NMR probe heads. Presented below are various approaches taken by the community (see also Figure 2.14).

\begin{tabular}{|c|c|c|c|c|c|}
\hline & Coil type & $\begin{array}{l}\text { Sample } \\
\text { volume [mL] }\end{array}$ & $\begin{array}{l}\text { Resolution } \\
\text { [ppb] }\end{array}$ & $\begin{array}{l}\mathrm{nLOD} \text { at } \\
600 \mathrm{MHz} \\
{\left[\mathrm{nmol} \mathrm{s}^{1 / 2}\right]}\end{array}$ & Reference \\
\hline a & Solenoid & 0.005 & 2 & 0.13 & Olson et al. ${ }^{12}$ \\
\hline b & Planar & 0.03 & 300 & 20.9 & Massin et al. ${ }^{13}$ \\
\hline c & Planar & 0.47 & 300 & 262 & Massin et al. ${ }^{13}$ \\
\hline d & Solenoid & $4 \times 10^{-8}$ & 1700 & 0.36 & Ciobanu et al. ${ }^{14}$ \\
\hline e & Planar & 0.393 & 12.4 & 13.8 & Trumbull et al. ${ }^{15}$ \\
\hline$f$ & Planar & 0.57 & 100 & 163 & Wensink et al. ${ }^{16}$ \\
\hline g & Stripline & 0.012 & 80 & 18.3 & $\begin{array}{l}\text { van Bentum et al. }{ }^{17} \\
\text { and Kentgens et al. }\end{array}$ \\
\hline h & Planar & 0.005 & 27 & 2.61 & Ehrmann et al. ${ }^{18}$ \\
\hline i & Planar & 0.00088 & 32 & 8.66 & Stocker et al. ${ }^{19}$ \\
\hline j & Solenoid & $6.4 \times 10^{-5}$ & 1700 & 16.5 & Badilita et al. ${ }^{20}$ \\
\hline k & $\begin{array}{l}\text { Phased } \\
\text { array }\end{array}$ & 0.77 & 11 & 7730 & Gruschke et al. ${ }^{11}$ \\
\hline I & Planar & 330 & 400 & 92000 & Renaud et al. ${ }^{21}$ \\
\hline $\mathrm{m}$ & $\begin{array}{l}\text { Planar } \\
\text { needle }\end{array}$ & 0.5 & 50 & 12.3 & Syms et al. ${ }^{22}$ \\
\hline$n$ & $\begin{array}{l}\text { Planar } \\
\text { Helmholtz }\end{array}$ & 10 & 20 & 125.6 & Goloshevsky et al. ${ }^{23}$ \\
\hline o & Solenoid & 0.03 & 2.33 & 141.1 & Rogers et al. ${ }^{7}$ \\
\hline $\mathrm{p}$ & Solenoid & 50 & 1000 & 30 & Yamauchi et al. ${ }^{24}$ \\
\hline$q$ & Planar & 1.2 & 7.5 & 22 & Ryan et al. ${ }^{8}$ \\
\hline r & Microslot & 0.025 & 1.8 & 7 & Maguire et al. ${ }^{10}$ \\
\hline s & Solenoid & 0.0001 & 2600 & 0.1 & Seeber et al. ${ }^{25}$ \\
\hline $\mathrm{t}$ & Microslot & 0.0106 & 7.2 & 0.39 & Krojanski et al. ${ }^{26}$ \\
\hline
\end{tabular}

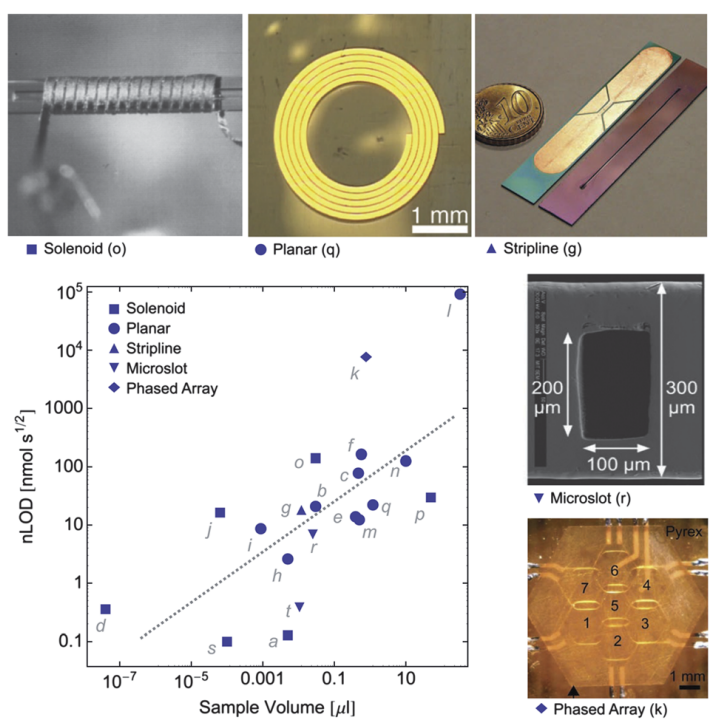

FIGURE 2.14: Left: Table of coil types and normalized limits of detection (LOD). Right: Normalized limit of detection of different $\mu$ NMR detection systems corresponding to the authors listed in table shown left. The nLOD values have been normalized and scaled to a $600 \mathrm{MHz}$ spectrometer. The dotted line indicates a power law with an exponent of 0.5 [64].

\subsubsection{Solenoid}

The approach first attempted for $\mu \mathrm{NMR}$ was to hand wind detector coil around a silica capillary [23] (shown Figure 2.14). Magnitude of order sensitivity gains were achieved from this simple first step, with resolution of $0.6 \mathrm{~Hz}$ recorded $^{10}$. Solenoid $\mu$ coils are very simple examples of achieving high filling factors. This fact drove Webb and Grant [65] to improve upon the filling factor by using thinner capillary walls. Ultimately, microfabrication techniques were applied for still more improved sensitivity and resolution results [66].

\footnotetext{
${ }^{10}$ This result was achieved by using per fluorocarbon FC-13, which has a magnetic susceptibility very close to copper, which was used as the coil material. In more typical LOC applications the microfluidic device material is the biggest contribution to susceptibility broadening.
} 
Solenoid design considerations (e.g. windings, wire spacing, and wire thickness) are now well defined in regards to optimization of RF-homogeneity and SNR [67, 68]. However, problems can be encountered when operating at higher frequencies since the wavelength of the RF signal starts to approach the dimensions of the solenoid coil [69].

\subsubsection{Linear}

The advantage of using a single current path - as is done with linear based coil designs instead of coils with windings, is that the sample will be exposed to a single current path magnetic field; which in theory should create a more homogeneous field than a typical helical coil. There are several variations of this method including stripline [11-13, 42], $\mu$ strip RF coil [70, 71], and $\mu$ slot geometries [15, 72] (configurations shown in Figure $2.14)$.

The operating principle of linear detectors is to create a standing wave half the wavelength of the Larmor frequency and orient the sample to the anti-node of the standing wave mode. Fabrication of linear detectors is less demanding than solenoids and they can achieve similar filling factors.

\subsubsection{Planar}

Due to the geometry of Planar $\mu$ coils $[7,9,28,73,74]$, integration with microfluidic devices becomes trivial using traditional lithographic methods. A slight improvement on this approach is Helmholtz planar pairs which offer improved homogeneity $[75,76]$ as compared with a single planar $\mu$ coil. The biggest advantage of planar $\mu$ coils is the 
flexibility in accommodating samples, which allows for a general protocol to be formed, since the chip and integrated resonator would be expendable.

\subsection{Conclusion}

The power of NMR to non-invasively provide detailed chemical information has led to it being the preferred tool of many areas of science, but current limitations on sensitivity prevent it from being applied to the micro- and nano- fluidic realm. While non-Boltzmann population methods present a possible solution, their lack of generalization prevent them from begin applied broadly. A alternative method is to improve RF-coil sensitivity. While smaller gains in sensitivity are obtained, they still allow for micro- and nano- fluidic NMR spectroscopy. 


\section{Chapter 3}

\section{Integrated Planar Microcoils for}

\section{Inductively Coupled NMR}

\subsection{Introduction}

NMR metabolomics in microfluidic systems requires optimal sensitivity. This means that the detector has to be adapted to the size and shape of the sample, which differs strongly from the cylindrical samples used in conventional NMR, both in shape and volume. Ease of experimental setup and low cost lend this method to applications in disposable microfluidic LOC devices. High filling factors are needed in microcoil applications, along with flexibility in sample switching. Inductively coupled resonators eliminate the need for electrical contact from the coil to the console and allow for high filling factor. The following chapter will present design considerations, fabrication methods, and coil characterization procedures. 


\subsubsection{Prior Work}

As mentioned in chapter 2.3.3.1 first attempts in developing microcoil RF-coils focused on simple hand made solenoids. Olson et al. reported 130 -fold gains in sensitivity and resolution of $0.6 \mathrm{~Hz}$ from a wire coil wrapped around a capillary. A drawback of this configuration is that it requires directly connected wire leads. This arrangement means the capillary has to be built into the probe head along with the microfluidic capillary. This restriction, runs contrary to the idea of self-contained disposable microfluidic devices; which would ideally be changed out of the spectrometer in rapid succession for point of care applications [77-79].

Jacquinot and Sakellariou addressed this problem by eliminating wire leads completely. They instead relied upon wireless coupling between a second small coil, installed to tightly engulf the sample, and the standard pickup coil equipped in the probe. Their interest was in solid-state rotating coils but the same principles can be applied to liquid state for small sample sizes.

In our group, we combine these two approaches to inductively coupled microfluidic devices in liquid state mode for metabolic study. As a first step, solenoid coils were developed for flow profiling. After successful demonstration using this method, planar microcoils were introduced for better integration to lithographically made microfluidic devices. Below is recapitulation of precedent work in the Utz group. 


\subsubsection{Velocity Profile}

Solenoid resonators were hand wound from magnetic wire and directly soldered to a non-magnetic capacitor. Using microimaging (see chapter 3.6.2) the flow velocity profile inside the capillary was measured (see Figure 3.1). The sensitivity and resolution recorded from this work, were comparable to directly coupled microcoils, suggesting that inductive coupling is a viable option for practical applications [16].

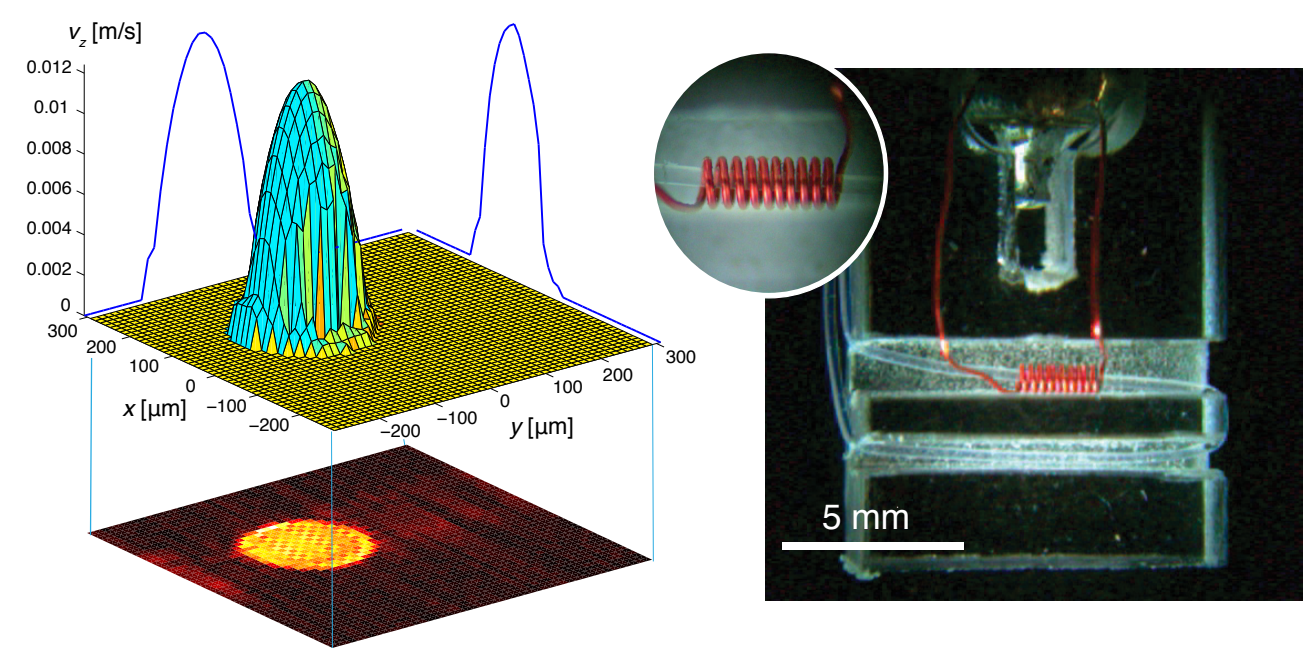

Figure 3.1: Left: Velocity profile compiled from individual displacement profiles at each pixel. Right: Experimental setup with micrograph of Teflon capillary and hand wound solenoid microcoil [16].

\subsubsection{Planar Microcoil Fabrication}

Flat helical coils are easily integrated with microfluidic systems since they are both fabricated using lithographic methods and thus represented the next step towards a fully self contained microcoil LOC device. Fabrication methods of glass based microfluidic devices and planar microcoils were established [16], shown in Figure 3.2. However, characterization of microcoils used directly connected wire leads, which presented characterization 
problems when moving from a directly connected bench-top network analyzer to a wireless NMR experimental environment. Uncompensated resistances were present in the wire leads, which distorted the self resonances of the coils as measured by the network analyzer, resulting in a mischaracterization. Ultimately, spectroscopic experiments were unable to produce reliable results from these mischaracterized microcoils.
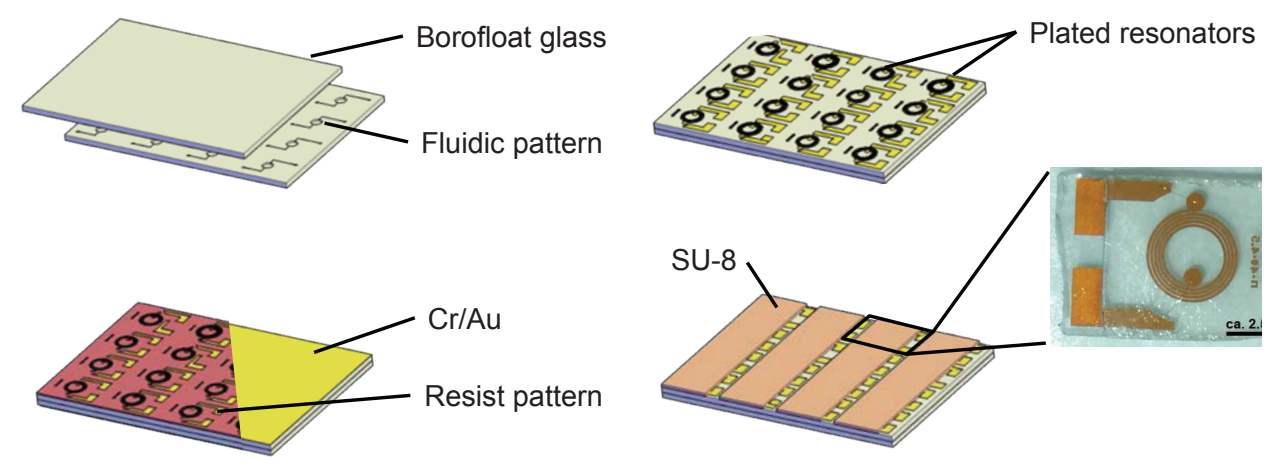

FIGURE 3.2: Fabrication schematic of LOC microfluidic device with integrated planar microcoil [80].

The work of this thesis focused on characterization methods which produced tuned 600 $\mathrm{MHz}$ ( $B_{0}$ operating frequency) microcoils for spectroscopic experiments in one and two dimensions.

\subsubsection{Experiment Configuration}

Figure 3.3 displays the probe head configuration with a typical microfluidic device with integrated microcoils. Based on the application, the LOC device could contain multiple sample chambers that would be of interest. Micro resonators could be placed at each site of interest and simultaneously analyzed spectroscopically. In practice, the factory made probe pickup coil was found to be incompatible with the geometry of even the most 


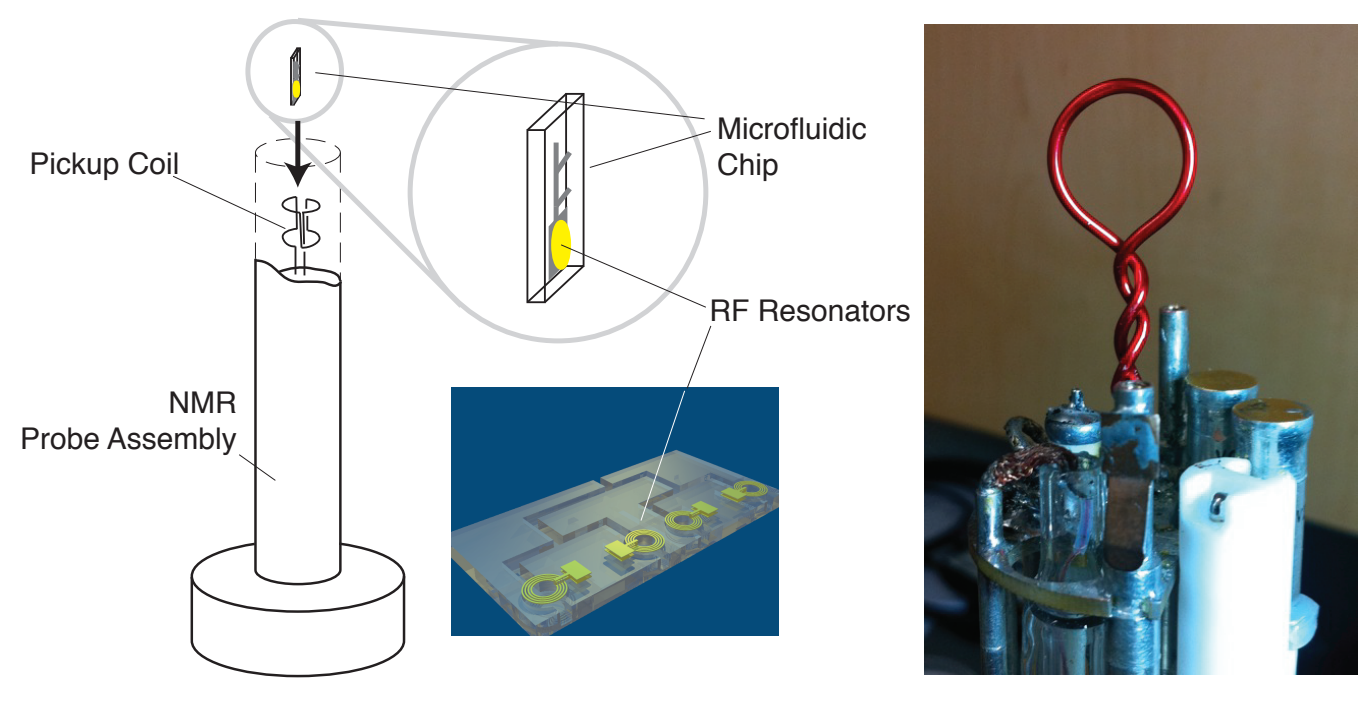

FiguRE 3.3: Left: Microfluidic inductive coupling experimental setup. Integrated microfluidic chip with RF resonator is placed in close proximity to the pickup coil inside the NMR probe; collectively, this is placed inside the bore of the NMR spectrometer [80]. Right: Early simple design of hand wound from magnetic wire, which will ultimately be inductively coupled to the microcoil on the chip.

simply microfluidic device and was replaced with a simple hand wound coil loop (shown on the right in Figure 3.3). The lower section of the coil is twisted together to constrain the magnetic field to the upper portion of the pickup coil, thus making alignment with the microcoil simpler.

The static magnetic field, $B_{0}$, is produced by a Varian 14.1 Tesla $(600 \mathrm{MHz})$ standard bore NMR magnet (see Figure 2.1). The superconducting magnet was manufactured by Oxford instruments and supplied to Varian (acquired by Agilent Technologies). For narrow linewidths in the NMR spectrum, the magnetic field should be homogeneous over the entire sample volume. Practically, this means that variations in the field should be better than one part-per-billion.

The NMR console includes RF-electronics to produce the $B_{1}$ field perpendicular to the static magnetic field, $B_{0}$. Figure 3.4 shows console electronics used in a NMR 
spectroscopy experiment.

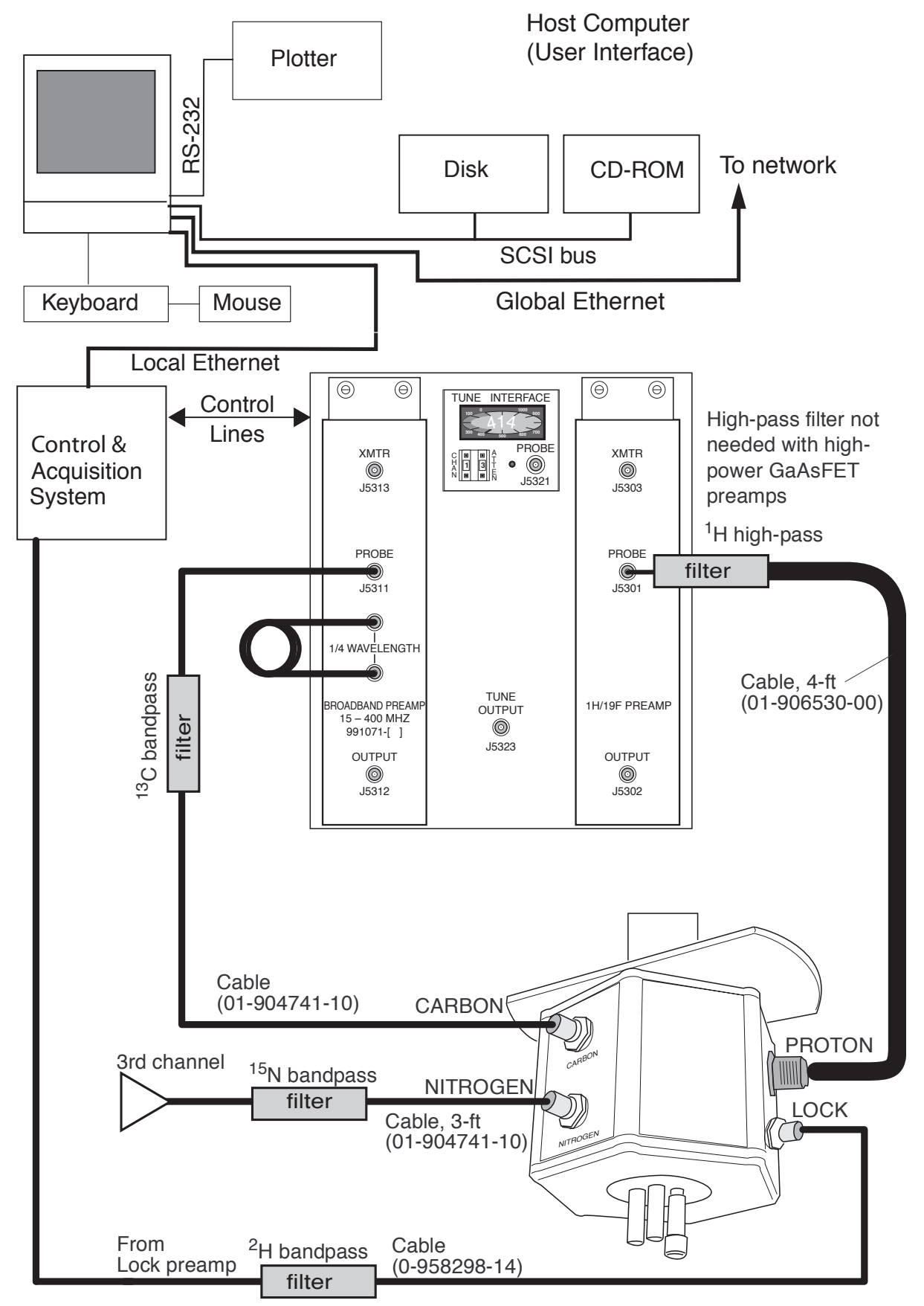

FiguRE 3.4: Schematic of NMR console hardware with experimental flow diagram $[81,82]$. 


\subsection{Sensitivity}

The SNR of a pulse NMR experiment can be described by the following relation $[19,83]$ :

$$
S N R \propto \frac{\frac{B_{R F}}{I} \omega_{0} M_{0} V}{\sqrt{4 k_{B} T R_{\text {coil }} \Delta f}}
$$

Where $B_{R F}$ is the RF magnetic field from the current $(I), V$ is the sample size, $M_{0}$ is the equilibrium magnetization from Equation 2.7, $\Delta f$ is the spectral bandwidth, and $R_{\text {coil }}$ is the resistance from the coil. The expression assumes an optimized filling factor, which is defined as the amount of the field occupied by the sample. Conversely, this means that the integral of the field area not occupied by the sample should be minimized. Equally important, is minimizing all losses from the resistance, $R_{\text {coil }}$. By holding the sample mass constant and including the magnetic field strength as a function of coil radius $(r)$, equation 3.1 can be reduced to [28]:

$$
S N R \propto \frac{1}{r}
$$

This simplified expression assumes the $B_{1}$ field from the coil is highly uniform across the sample. With this assumption satisfied the SNR behaves inversely proportional to the coil radius. Implicit within Equation 3.2, is that as the coil volume shrinks so must the size of the sample and correspondingly so must the number of spins. 


\subsubsection{Limit of Detection}

Experimental SNR can be problematic method of measurement, since it can depend on both equipment configuration and post-processing treatment. The standard by which NMR probe sensitivity is measured is called limit of detection (LOD). The time-domain limit of detection, is defined as the number of spins required to resonate in a $1 \mathrm{~Hz}$ bandwidth to give a signal equal to the noise, which can be written [11]:

$$
\operatorname{LOD}_{t}=\frac{N_{S}}{\operatorname{SNR}_{t} \sqrt{\Delta f}}
$$

where $N_{S}$ represents the number of protons contributing to the signal, and $\Delta f$ is the width of the resonance line. The sample used to determine the LOD must have a well characterized concentration of protons. The total number of protons in the sample is then easily obtained from the first data point in the FID (see Figure 2.7).

\subsubsection{Planar Coils}

Spiral planar coils represent a convenient fabrication style in regards to integration on microfluidic devices. This is because LOCs are typically constructed using microelectronic methods of deposition of conducting material, patterning with photoresist, and chemically etching the desired design. Equation 3.1 has been applied to planar microcoils using Bio-Savart's law, and expressions for $B_{R F} / I$ and $R_{\text {coil }}$ were derived, assuming a infinitely thin conductor $[9,84]$ : 


$$
\frac{B_{R F}(0, z)}{I}=\frac{\mu_{0}}{2 \pi \Delta}\left(\frac{1}{\sqrt{\left(\frac{z}{r_{0}}\right)^{2}+1}}-\frac{1}{\sqrt{\left(\frac{z}{r_{0}}\right)^{2}\left(\frac{r_{0}}{r_{f}}\right)^{2}+1}}+\ln \left[\frac{r_{f}}{r_{0}} \frac{1+\sqrt{\left(\frac{z}{r_{0}}\right)^{2}\left(\frac{r_{0}}{r_{f}}\right)^{2}+1}}{1+\sqrt{\left(\frac{z}{r_{0}}\right)^{2}+1}}\right]\right)
$$

where $z$ is the axial distance from the coil plane to the observation point, $\mu_{0}$ is the magnetic permeability of free space, $r_{0}$ is the inner radius, $r_{f}$ is the radius to the outer most coil turn, and $\Delta$ is the turn spacing. Note that Equation 3.4 and 3.5 are derived for a ideal single coil with a current, not for the inductively coupled case. For a microcoil with some thickness, $t$, and some width, $w$, we assume that both these dimensions are greater than the skin depth, $\delta$. The $\mathrm{AC}$ coil resistance is:

$$
\begin{aligned}
R_{\text {coil }} & =\frac{\rho}{4 \delta(w+t-2 \delta)} \\
& \times\left(r_{f} \sqrt{1+\left(\frac{2 \pi r_{f}}{\Delta}\right)^{2}}-r_{0} \sqrt{1+\left(\frac{2 \pi r_{0}}{\Delta}\right)^{2}}+\frac{\Delta}{2 \pi} \ln \left[\frac{r_{f}}{r_{0}} \frac{1+\sqrt{\left(\frac{\Delta}{2 \pi r_{f}}\right)^{2}+1}}{1+\sqrt{\left(\frac{\Delta}{2 \pi r_{0}}\right)^{2}+1}}\right]\right),
\end{aligned}
$$

where $\rho$ is the resistivity. The skin depth $(\delta)$ is frequency dependent:

$$
\delta=\sqrt{\frac{\rho}{\pi \mu_{0} f}}
$$

If equations 3.4 and 3.5 are substituted into equation 3.1 the expression for the SNR per unit volume is derived. Figure 3.5 shows the expression for SNR for a microcoil versus the normalized distance form the coil plane. Improved SNR ratios are predicted for samples that can be closely confined to the region near the microcoil. In cases when static capillary are unacceptable, such as point of care disposable devices, a secondary 
microcoil is integrated onto the surface of the microfliudic device and is inductively coupled to the larger pickup coil.

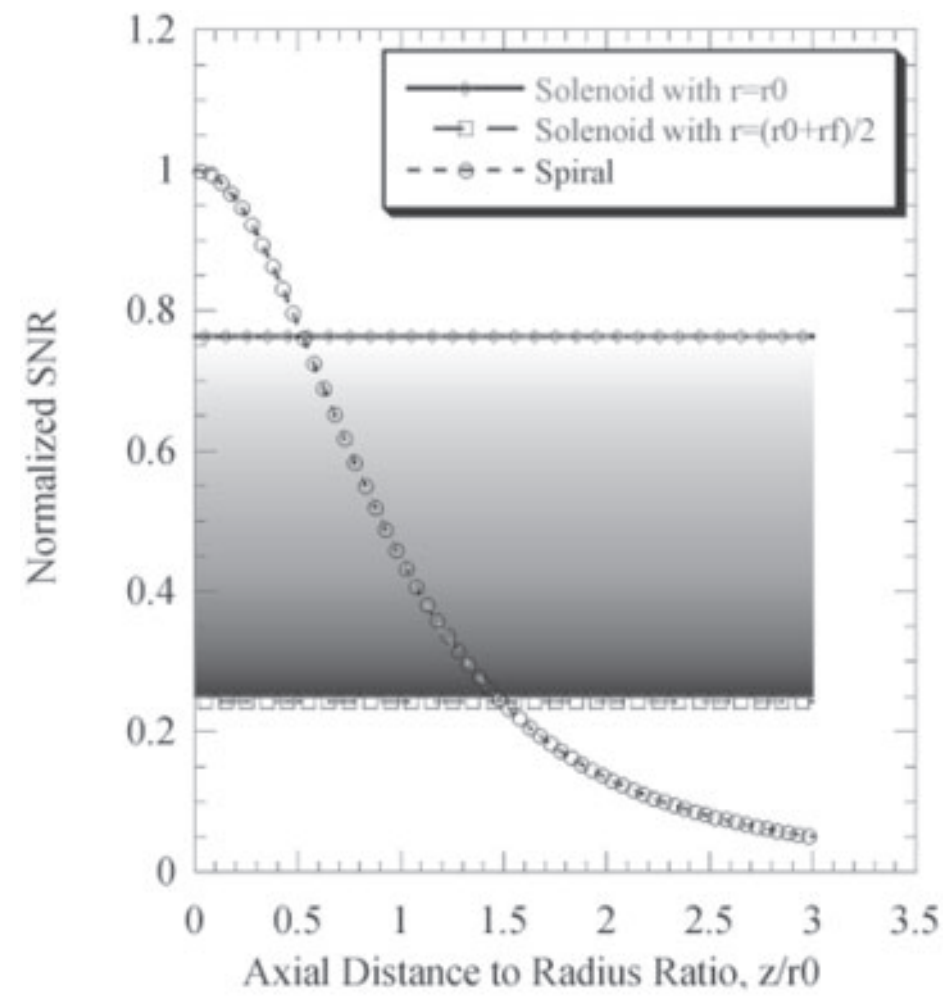

FIGURE 3.5: Normalized SNR per unit volume versus axial distance to starting radius ratio for solenoid and planar coil configurations. This graph predicts that the SNR ratio for a planar coil is better than a solenoid when in close proximity to the sample volume [84].

\subsubsection{Cost of Inductive Coupling}

Figure 3.6 shows the effective circuit diagram of a micro resonator (orange) inductively coupled to a conventional NMR probe (blue) and the sensitivity obtained from the system given various quality factors (Q-factors) of the micro resonator.

A simplified expression for the SNR of the probe, directly coupled microcoil system, and inductively coupled microcoil system can be written: 

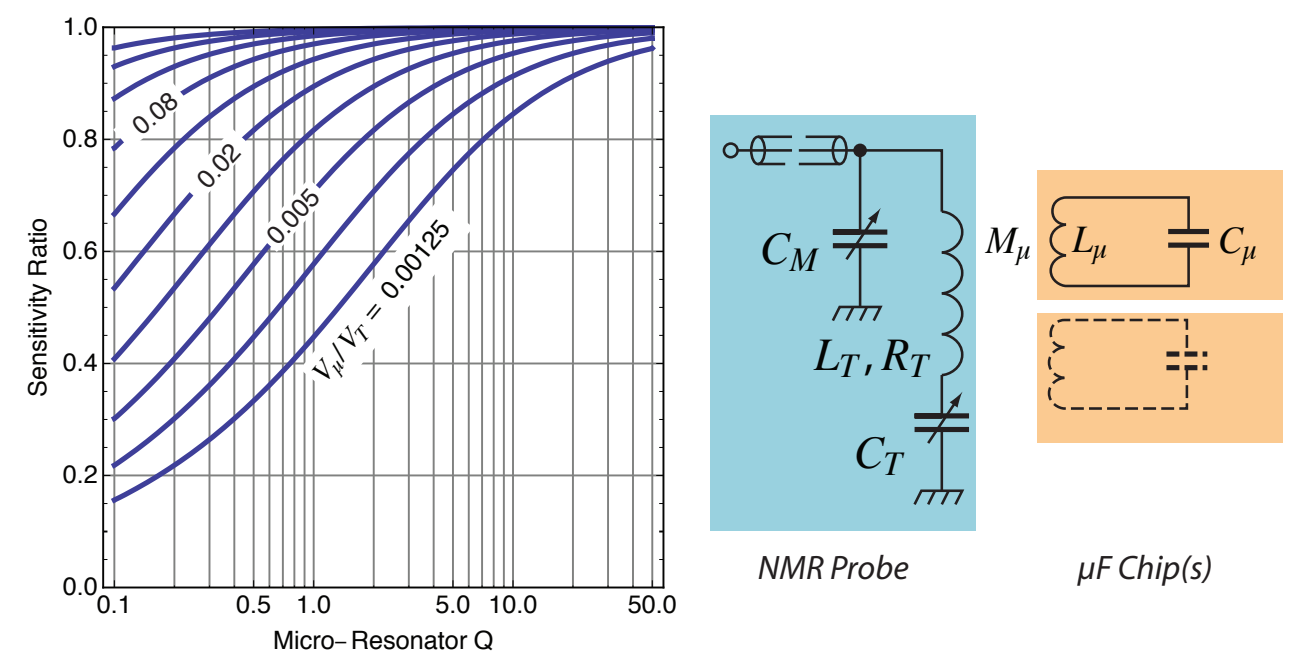

FIgURE 3.6: Left: Ratio of sensitivity of inductively coupled over direct microcoil NMR on the vertical axis. On the horizontal axis is the quality factor of the micro resonator. Several curves defined for various volume ratios $\left(V_{\mu} / V_{T}\right)$ [84]. Right: Modeled inductively coupled system in the probe head.

$$
\begin{aligned}
& S N R_{\text {probe }} \approx B_{T} / I_{T} \sqrt{R_{T}} \\
& S N R_{\text {direct }} \approx B_{\mu} / I_{\mu} \sqrt{R_{\mu}} \\
& S N R_{R F} \approx B_{\mu} / I_{T} \sqrt{R_{T}}
\end{aligned}
$$

Sensitivity is gained from the inductively coupled microresonator by the ratio $B_{\mu} / B_{T}$ and can be written:

$$
\frac{S N R_{R F}}{S N R_{\text {probe }}}=\frac{B_{\mu}}{B_{T}}=Q_{\mu}
$$

where $Q_{\mu}$ is the quality factor of the microresonator. Comparing this equation to the directly coupled system we have:

$$
\frac{S N R_{R F}}{S N R_{\text {direct }}}=\frac{k \sqrt{Q_{T} Q_{\mu}}}{\sqrt{1+k^{2} Q_{T} Q_{\mu}}}
$$


This expression represents the cost of using a inductively coupled system and is plotted in Figure 3.6, where $k^{2}$ is $V_{\mu} / V_{T}$. From Figure 3.6 that even for the smallest volume ratio (0.00125) the sensitivity ratio will exceed $90 \%$ so long as the microcoil has a $\mathrm{Q}$ value above 25 . While there may be a very small sacrifice in sensitivity, the flexibility of this platform makes it a more attractive option for integration with LOC devices.

\subsection{Resolution}

In practice, sensitivity gain using microresonators is straightforward in that it requires coils with well resolved features on the micro-scale level positioned close to the sample of interest. A less obvious problem to solve is that of spectral resolution. Differences in susceptibility between coil material, coil substrate, sample contents, and most importantly chip material cause variations in the field close to the region of interest. For example, Figure 3.7 shows the many interfaces and different materials present in the immediate area around the microfluidic LOC device when loaded into the NMR probe head. Example magnetic susceptibility values are listed in table 3.1.

In traditional NMR spectroscopy, magnetic field inhomogeneities are compensated by shimming, which is the process of homogenizing the magnetic field by passing current through coils near the sample region. However, shims are large and relatively far away from the microresonated sample, making them less effective. Another approach avoids inhomogeneities from the start by advantageously placing materials depending upon their magnetic susceptibility. An example would be to replace the air present in the probe volume with a fluorocarbon which has the same susceptibility as copper [23, 85]. 

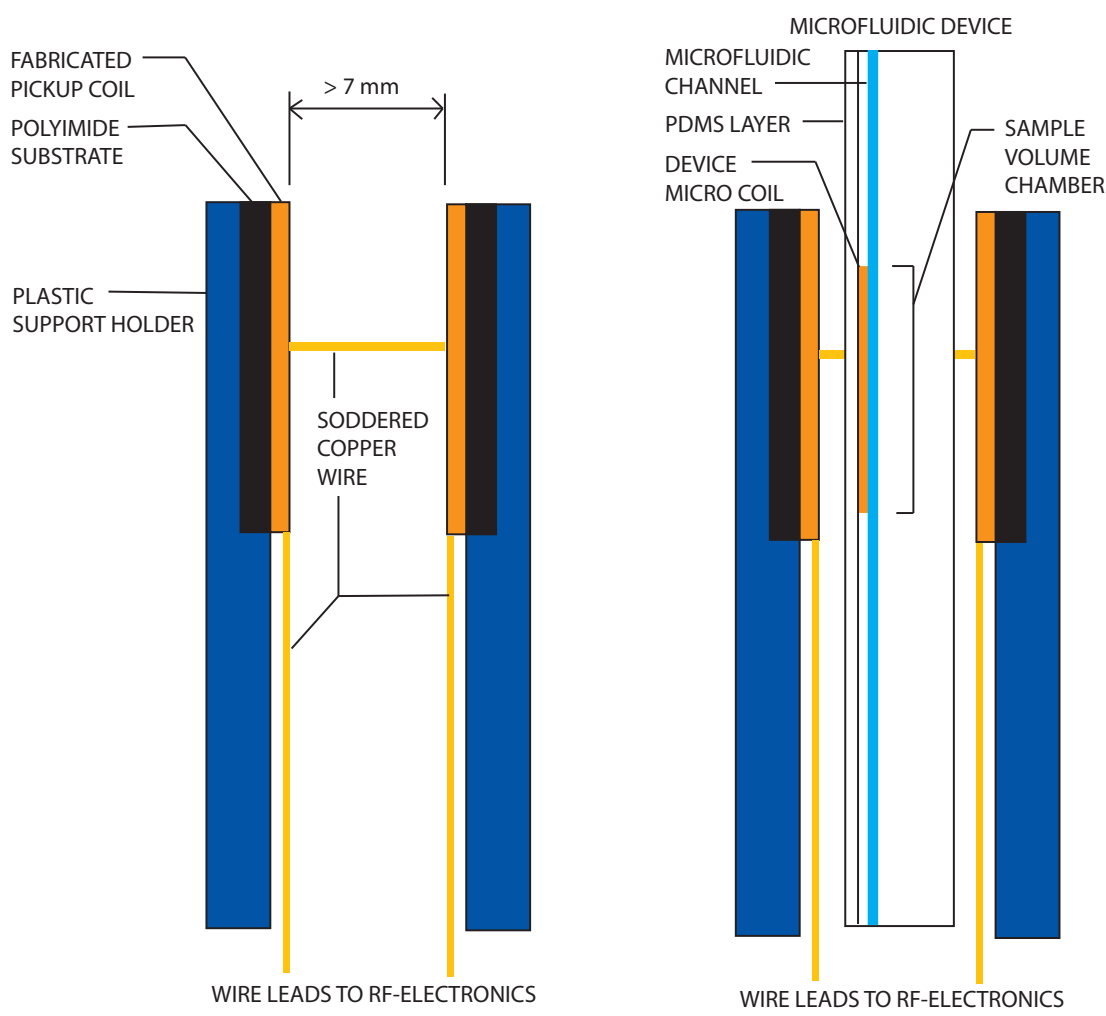

FiguRE 3.7: Left: Profile image of unloaded sample holder with pickup coil and probe support structure. Right: Profile of sample holder loaded with microfluidic device.

This would produce no susceptibility jumps, as the magnetic field would behave as if the fluorocarbon and copper were a continuous object, and thus produce less variation in the field.

TABle 3.1: Magnetic Susceptibilities $\left(20^{\circ}\right.$ C) $[86,87]$

\begin{tabular}{|l|l|l|}
\hline Material & Volume Susceptibility $\left(\chi_{m}\right)$ & Density \\
\hline Units & at $7 \mathrm{~T}$ & $\mathrm{~kg} / \mathrm{m}^{3}$ \\
\hline Water & $-9.05 \times 10^{-6}$ & 997 \\
\hline $\mathrm{Al}_{2} \mathrm{O}_{3}$ & $-14 \times 10^{-6}$ & 3950 \\
\hline Vespel & $-9.2 \times 10^{-6}$ & 1430 \\
\hline Quartz & $-11.8 \times 10^{-6}$ & 2250 \\
\hline Teflon $(\mathrm{PTFE})$ & $-10.5 \times 10^{-6}$ & 2200 \\
\hline Silicone & $-7.8 \times 10^{-6}$ & 1080 \\
\hline Pyrex-7070 & $-11 \times 10^{-6}$ & 2500 \\
\hline
\end{tabular}


Incorporating exotic materials into the probe design, such as ${ }^{129}$ Xe for hyperpolarization or Fluorinert FC-43 for susceptibility matching, can be expensive and a time consuming engineering challenge. Therefore, it should be regarded as secondary option. Another alternative focuses on the alignment of the microfluidic, microresonator, and probe structural components in regards to the static $B_{0}$ field [88]. Specifically, boundaries between materials should be avoided where possible, but where unavoidable should be constructed so they are completely vertical in the sample region. Much like the infinite wire oriented parallel to a magnetic field (see chapter 2.3.3.2), other physical structures effects are decreased when oriented parallel to the field. This methodology can help guide the design of the microfluidic device and probe structure.

\subsection{Design}

Prior to microresonator design for the work presented in this thesis, detailed probe and pickup coil characteristics had to be researched and recorded. Initially, probe pickup coils with self resonances at the Larmor frequency (600 MHz) were designed and fabricated; however, early empirical results showed this a faulty approach for the inductively coupled case. Subsequent modeling was performed to elucidate the theoretical optimum probe self resonance (discussed in Chapter 3.4.1).

Initial prototypes using the Helmholtz configuration ${ }^{1}$ gave spurious signals and were replaced in favor of a simple induction loop (see Figure 3.3). Figure 3.8 shows two prototype Helmholtz coils and their ultimate successor, the induction loop. Microresonator

\footnotetext{
${ }^{1}$ The Helmholtz configuration is a pair of circular magnetic coils that are symmetrically separated by the radius of the coils. The sample would be placed between the two coils. A bridge connection must be made between the first and second coil.
} 
TABle 3.2: Genisis of Microcoil Design

\begin{tabular}{|c|c|l|l|}
\hline Coil Type & Coil Image & Description \\
\hline Wire Solenoid & & $\begin{array}{l}\text { Tunable solenoid wrapped } \\
\text { around a capillary }\end{array}$ \\
\hline Planar & & $\begin{array}{l}\text { First glass planar coil with in- } \\
\text { tegrated capacitance }\end{array}$ \\
\hline Planar & & $\begin{array}{l}\text { Glass based planar coil with } \\
\text { capacitance built into wind- } \\
\text { ings }\end{array}$ \\
\hline Tunable Planar & $\begin{array}{l}\text { Copper/polyimide foil tun- } \\
\text { able planar coil }\end{array}$ \\
\hline Planar & $\begin{array}{l}\text { Current generation cop- } \\
\text { per/polyimide foil planar coil } \\
\text { with comb capacitor }\end{array}$ \\
\hline
\end{tabular}

design and inductive coupling theory are presented heretofore.

\subsubsection{Inductive Coupling}

The theory of inductively coupled NMR detectors has been well described in the literature with a comprehensive review given by Jacquinot and Sakellariou [17], so that only key points need to be summarized here. Figure 3.9 demonstrates the principle. The resonator is modeled as a tank circuit, with an inductance $L_{\mu}$, capacitance $C_{\mu}$, and parasitic resistance $R_{\mu}$. The resistance $R_{\mu}$ consists of both a internal ohmic resonator resistance and a dielectric loss in the sample. The Probe coil has inductance $L_{T}$ and 

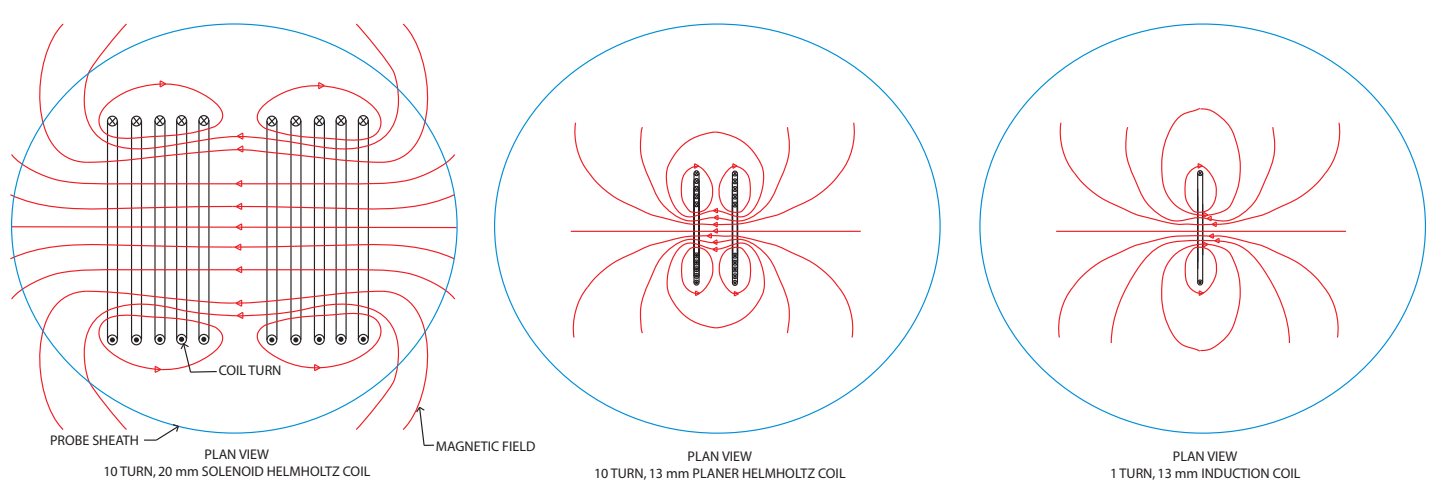

Figure 3.8: Left: Standard solenoid Helmholtz coil. Middle: Planar Helmholtz design, showing improved magnetic field around the area of the sample. Filling factor is still poor in this configuration compared with capillary solenoid microNMR. Right: A simple induction loop close the sample still doesn't have a filling factor neat 0.5 but is ultimately a simple design to implement with a self resonance far away from the chip microcoil.

parasitic resistance $R_{T}$. Finally, the tuning and matching network consists of capacitors $C_{T}$ and $C_{M}$.

The correspondence principle states that the sensitivity of inductively coupled NMR is proportional to the amplitude of the oscillating magnetic field at the sample location cased by an oscillating current of unit magnitude [19]. The parasitic resistances of the probe and the resonator, typically represent the dominant sources of noise. Optimum sensitivity is reached when the fraction of power being dissipated in the resonator is maximized. This corresponds to a minimization of the fraction of power being dissipated in the pickup coil.

Figure 3.9A shows the simple circuit used to modeled the system by linear network analysis. The dissipated fraction of power in the resonator can be expressed as the normalized angular frequency:

$$
\Omega=\omega / \omega_{\mu},
$$



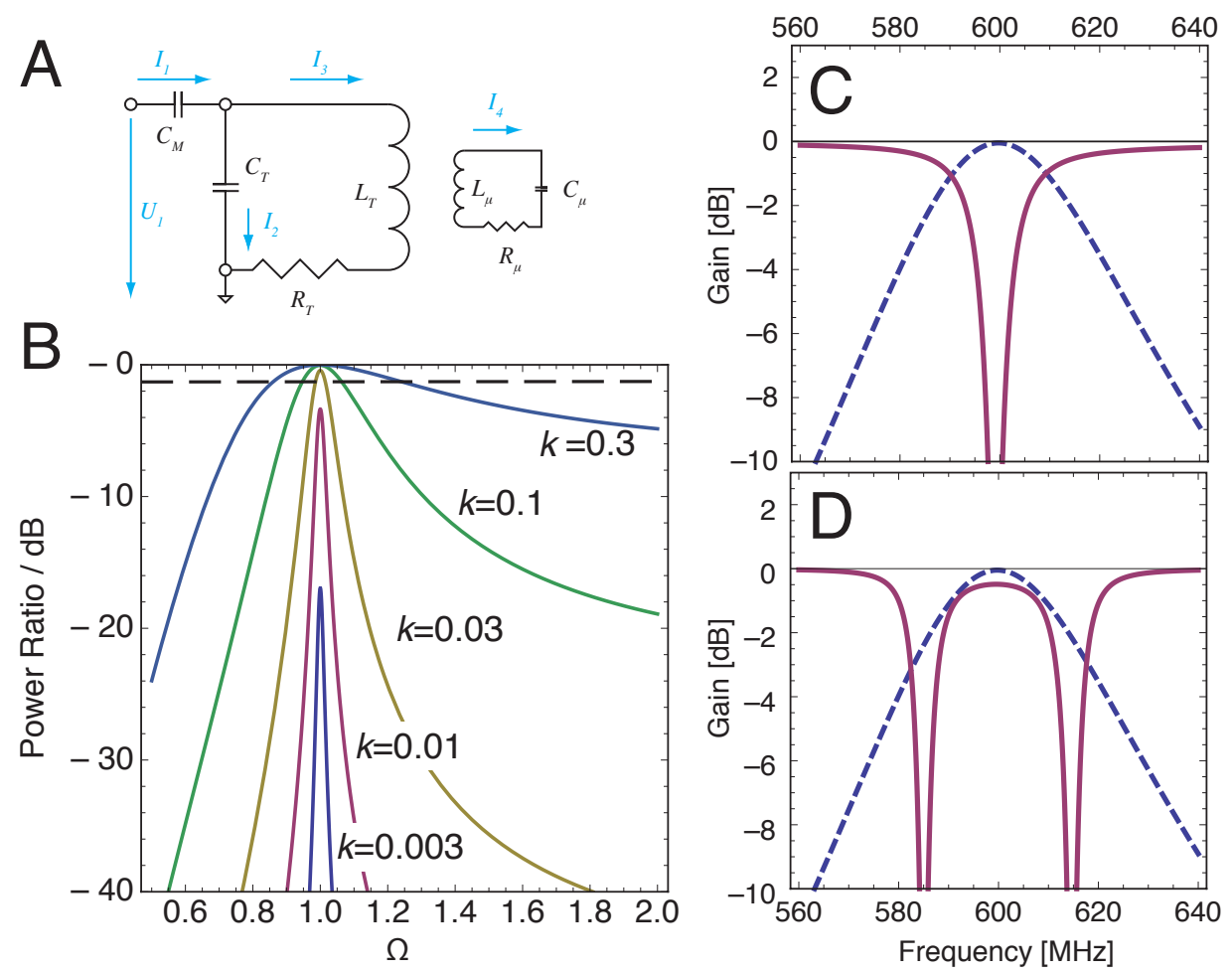

Figure 3.9: A: Basic circuit diagram. B: Power efficiency ${ }^{2}$ as a function of normalized frequency $\Omega$ and coupling constant $k$. C: Reflection ratio (solid line) and power efficiency (dotted line), calculated for a resonator at $600 \mathrm{MHz}$ with a $Q$ factor of 50 and $k=$ 0.05. The probe circuit is not tuned $\left(C_{T} \approx 0\right)$. D: Probe coil tuned by a finite $C_{T}$ value to $600 \mathrm{MHz}$. The reflection minimum resonance splits into two lines, separated by $k v_{0}$, where $v_{0}$ is the resonance frequency [73].

where $\omega_{\mu}$ is the nominal resonance frequency of the micro-resonator ${ }^{3}$, written as:

$$
\omega_{\mu}=1 / \sqrt{L_{\mu} C_{\mu}}
$$

The mutual inductance $(M)$ between the probe and the resonator is expressed as:

$$
M=k \sqrt{L_{T} L_{\mu}}=k / \omega_{\mu}
$$

which is characterized by the coupling constant, $k$. The power efficiency, which is the fraction of the total power applied that is dissipated in the microcoil, for the system is written in the following form:

\footnotetext{
${ }^{3}$ The nominal resonance frequency of the micro-resonator should operate at the Larmor frequency of the magnet (i.e. $\omega_{\mu}=\omega_{0}$ ).
} 


$$
\eta=\frac{k^{2} Q_{\mu} Q_{T} \Omega^{4}}{\Omega^{4}+k^{2} Q_{\mu} Q_{T}+Q_{\mu}^{2}\left(\Omega^{2}-1\right)^{2}}
$$

where $Q_{T}$ and $Q_{\mu}$ are the respective quality factors of the probe and the resonator. This equation is plotted on a logarithmic (dB) scale in Figure 3.9B for $Q_{T}=500$ and $Q_{\mu}=$ 50 , where the $-3 \mathrm{~dB}$ level is indicated with a dashed line ${ }^{4}$. Small $k$ values display peak widths of $1 / Q_{\mu}$. For $\Omega=1$ we see power efficiency peak maximums, which are given by:

$$
\eta_{\max }=\frac{k^{2} Q_{\mu} Q_{T}}{k^{2} Q_{\mu} Q_{T}+1}
$$

As $k$ increases, the peak approaches unity [17], which is described by the equation:

$$
k>k_{\text {crit }}=1 / \sqrt{Q_{\mu} Q_{T}}
$$

In the over coupling regime (i.e. $k \gg k_{c r i t}$ ), only a small part of the power is dissipated in the probe coil, resulting in a broadening of the power efficiency peak. In the case of concentric solenoids, $k$ is the square root of the ratio of the coil volumes. This can also be a useful approximation for other geometries.

In Figures 3.9C and 3.9D the predicted probe efficiency (dashed line) and the reflection ratio at optimum matching (solid line) are shown for a planar microcoil. In Figure 3.9D the probe tuning capacitance $C_{T}$ was adjusted so the probe circuit resonated at 600 MHz. Figure 3.9C set the tuning capacitance to a small number, resulting in essentially all power being dissipated in the resonator since the matching condition and efficiency

\footnotetext{
${ }^{4}$ Below the $-3 \mathrm{~dB}$ line, more than half of the input power is dissipated in the probe coil and not the resonator
} 
curve coincide (see Figure 3.10). If however, both the resonator and the probe are tuned to $\omega_{0}$, the matching curve splits into two peaks and causes half of the power/sensitivity being lost in the probe. Therefore, the non-tuned coupled mode was chosen for the experimental setup 5 .

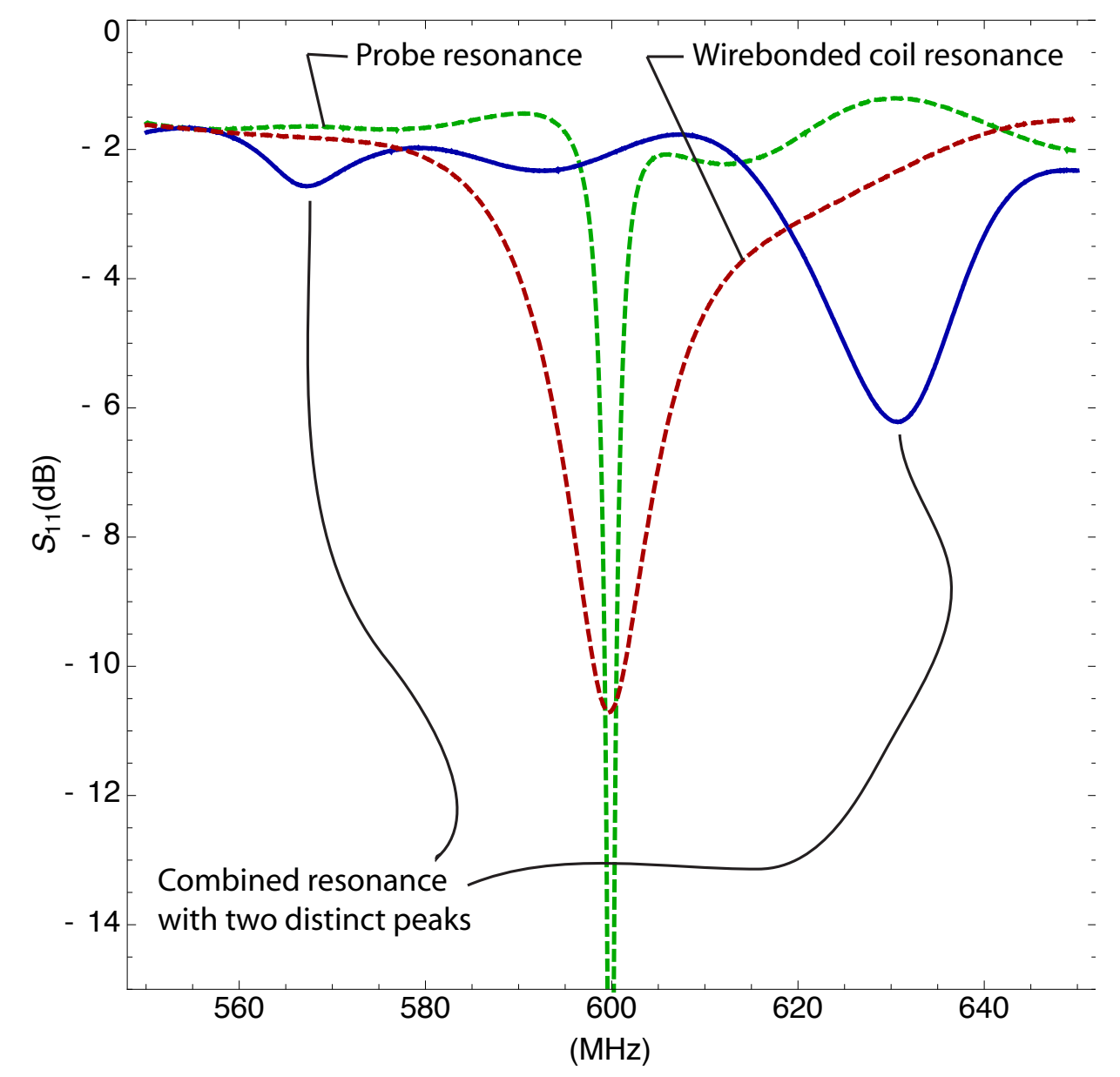

Figure 3.10: Experimental tuning curves for a individual microresonator (dashed red), probe (dashed green), and the combined tuning curve of the resonator and probe showing a split coupling situation (blue).

\footnotetext{
${ }^{5}$ The mathematica notebook with inductive coupling calculations is presented in Appendix A.
} 


\subsubsection{Microresonator Design}

As mentioned in chapter 3.1.1.2, previous efforts to record NMR spectra experimentally were unsuccessful due to a defective approach to on-bench resonance measurement, causing coil mischaracterization and compounded by fabrication errors. To address this issue tunable capacitors were incorporated into the next generation design of microcoils ${ }^{6}$. However, while this method could achieve the correct operating frequency, it sacrificed resolution by introducing susceptibility broadening material, present in the capacitor. Follow up work focused on moving linebroadening structures on the coil (such as the capacitors), away from the sample region. Figure 3.15 shows a fabricated tunable coil.
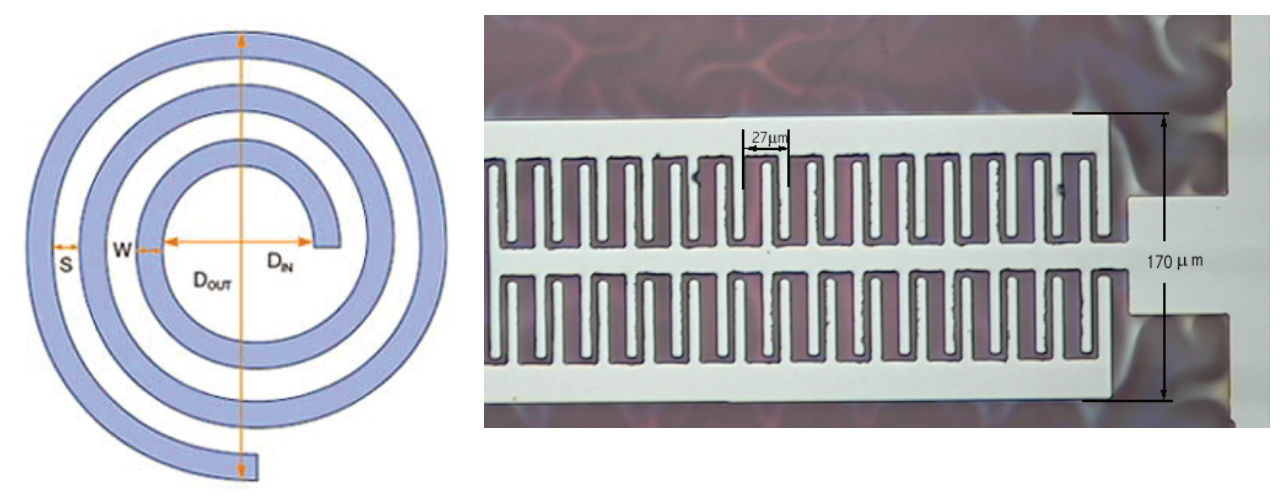

Figure 3.11: Left: Typical planar coil used for inductance calculations. Right: Adjustable comb capacitor in microelectronics circuit [90]. Each alternating finger is of opposite charge and creates a miniature capacitance, which can be added to by adding additional fingers.

Fixed comb capacitors (Figure 3.11) were integrated into the design since they introduce minimal susceptibility artifacts but still allow the microresonator to be tuned by crudely removing fingers of the comb [91]. Design specifics of microcoils incorporating comb capacitors, were derived from the Wheeler formula [92, 93]:

\footnotetext{
${ }^{6}$ Badilita et al. had previously shown that tunable resonators were a viable option for imaging [89].
} 


$$
L=\frac{r^{2} A^{2}}{30 A-11 D_{i}}, \quad \text { where } A=\frac{D_{i}+N(w+s)}{2}
$$

$r$ is the radius, $D_{i}$ is the inner diameter, $N$ is the number of turns, $w$ is the turn width, and $s$ is the turn spacing. A baseline inductance $(L)$ was measured from the tunable microcoils (shown Table 3.2) and deviated from as space restrictions allowed. The predicted frequency of the total system was calculated from:

$$
f_{R}=\frac{1}{2 \pi \sqrt{L C}}
$$

where $C$ is the measured capacitance; in this case, from the comb capacitor which can be expressed as:

$$
C=N_{f} \frac{\epsilon_{0} \epsilon_{r} t x_{0}}{d}
$$

$N_{f}$ is the number of fingers, $\epsilon_{0}$ is the permeability of the vacuum, $\epsilon_{r}$ is the permeability of the substrate, $t$ is the thickness of the finger, $x_{0}$ is the overlap distance, and $d$ is the distance from finger to finger. Full calculation Mathematica notebooks are available in Appendix B.

A secondary design criterion was to place the capacitors as far from the sample region as permitted by the size restraints of the microfluidic chip. The final chip design can be seen in Figure 3.12 . 


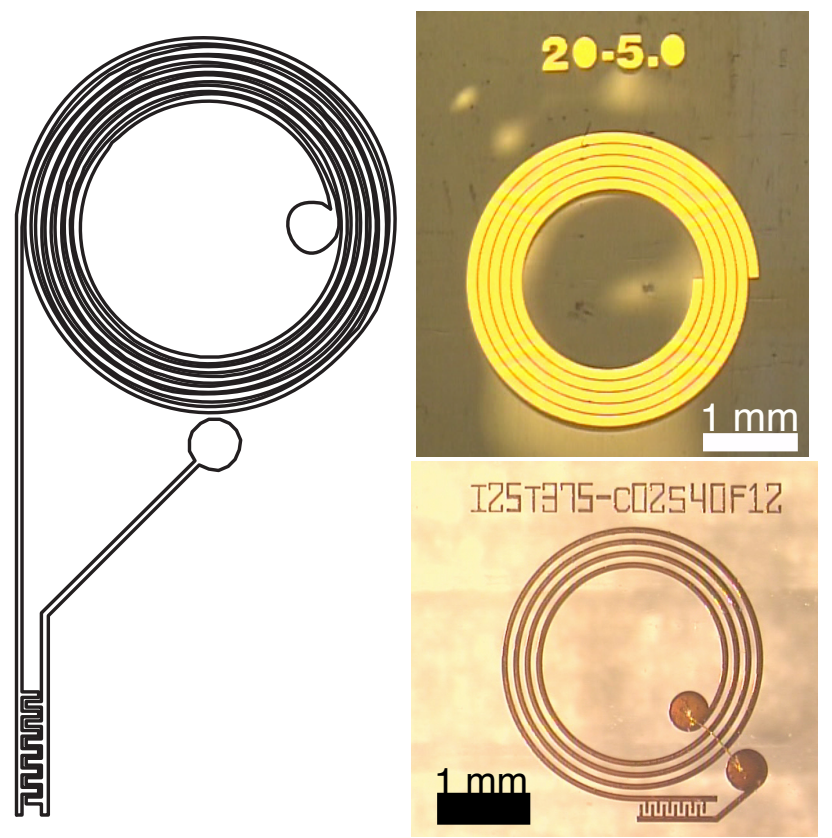

FiguRE 3.12: Left: AutoCAD drawings of redesigned microcoil with comb capacitor. Right: Two previous versions of microcoil which were not tuned correctly.

\subsection{Fabrication Methods}

The calculations described in Chapter 3.4.2 are approximations and accordingly the fabricated microcoils will be a best guess. To avoid many redesigns, multiple iterations with varying coil turns, turn thickness, turn spacing, and number of capacitor fingers were created. The complete set of design masks can be seen in Appendix C. Another obstacle in cleanly creating microresonators is the fabrication process itself. Although lithography is precise enough to create nano-level features, human error and inconsistent materials can cause irregularities to the final product. In this section, past and present fabrication methods will be reviewed for microfluidic and microresonator components. 


\subsubsection{Fluidic Channels}

Microfluidic devices were fabricated using either a glass or poly(methylmethacrylate) substrate. Inexpensive material costs for PMMA based microfluidics have made this a increasingly popular substitute for glass [94-96]. Fabrication characteristics of common material substrates are listed in Table 3.3. Specific fabrication details for glass and PMMA are presented heretofore.

TABLE 3.3: Substrate Fabrication Method Comparison [97]

\begin{tabular}{|l|l|l|l|}
\hline Substrate & Fabrication Method & Bonding Method & Fabrication Time \\
\hline Silicon & Reactive ion etching & Anodic & $8-10 \mathrm{~h}$ \\
\hline Glass & Chemical etching & Thermal or UV adhesive & $1-2$ days \\
\hline COC & Milling & Thermal & $6-8 \mathrm{~h}$ \\
\hline PC & Injection molding & Thermal & $6-8 \mathrm{~h}$ \\
\hline PDMS & Lithography & Plasma surface modification & $2-4 \mathrm{~h}$ \\
\hline PMMA & Laser ablation & Thermal or solvent & $1-2 \mathrm{~h}$ \\
\hline
\end{tabular}

\subsubsection{Glass Substrate}

Initially, borofloat glass sheets were sputtered with $\mathrm{Cr}$, and spin coated with a layer of AZ 1600 photoresist. However, this method was time consuming and had quality control issues. Blank chrome photomasks were instead purchased from TELIC Co. (Valencia, CA). The fluidic pattern was drawn in AutoCAD, uploaded to a Heidelberg DWL66 direct laser writer for photomask exposure (resolution of $4 \mu \mathrm{m}$ ), and finally developed using AZ 400K developer solution diluted in deionized water in a 1:4 ratio, respectively. Chromium remover was then used to expose the glass substrate in the areas patterned. Fluidic channels were etched with into the borofloat glass, in these areas, to a depth of $150 \mu \mathrm{m}$ using hydrofluoric acid. Access ports at each end were drilled into the substrate 
using diamond drill bits (acetone was used to remove the remaining photoresist). To remove fine glass particles and dirt from the surface, the fluidic channels were washed in an ultrasonic cleaning bath. The chips were then covered by a second glass layer, which was thermally fusion bonded.

\subsubsection{Polymer Substrate}

Bulk PMMA sheets were purchased from Astra Products (Aurora, OH), $200 \mu \mathrm{m}$ in thickness. Fluidic channel designs, drawn in AutoCAD, were translated to the PMMA using laser ablation by a VersaLASER 3.50 (Universal Laser Systems, Scottsdale, AZ). A bottom bottom blank layer and a top cover layer, with access ports, were also cut from PMMA; the three layers were then chemically bonded together using a low azeotropic solvent developed by Lin et al. [94, 98]. The solution - 20\% 1,2-dichloroethane (DCE), by weight, $80 \%$ ethanol - was pipetted on the surfaces of each layer and along the contact edges. The chip was then placed in a clamping device and allowed to rest for $\approx 1 \mathrm{~h}$. The total chip thickness was $600 \mu \mathrm{m}$, with a channel $200 \mu \mathrm{m}$ deep.

\subsubsection{Microcoil Lithography}

Planar NMR resonators were fabricated by two different lithographic methods, either by

(i) deposition and patterning of gold onto blank photoresist masks or by (ii) patterning of copper/polyimide flexible sheets. For completeness, both methods will be reviewed. 


\subsubsection{Glass Deposition}

Blank TELIC borofloat chromium masks were sputtered with a $50 \mathrm{~nm}$ thick gold seed layer after the surface was cleaned with deionized water and acetone. A $16 \mu \mathrm{m}$ layer of $\mathrm{AZ} 4500$ positive photoresist was spin coated on to the gold surface. Coil designs, were again handled in AutoCAD and transferred to the specimen by a direct laser writer. After photoresist development and $\mathrm{O}_{2}$-plasma cleaning, the coil structure was electroplated in Au up to a additional thickness of $7 \mu \mathrm{m}$. All photoresist was then removed with dimethyl sulfoxide. The remaining gold seed layer was stripped by aqua regis etching. At this stage, a gold wire bonder was used to make the bridge connection needed to complete the circuit. This connection was out of plane and as a protective measure a $10 \mu \mathrm{m}$ layer of SU-8 was spin coated onto the resonator. The complete process is shown in Figure 3.13 with a simplified version shown in Figure 3.2. A coil fabricated using this method is shown in Figure 3.12.
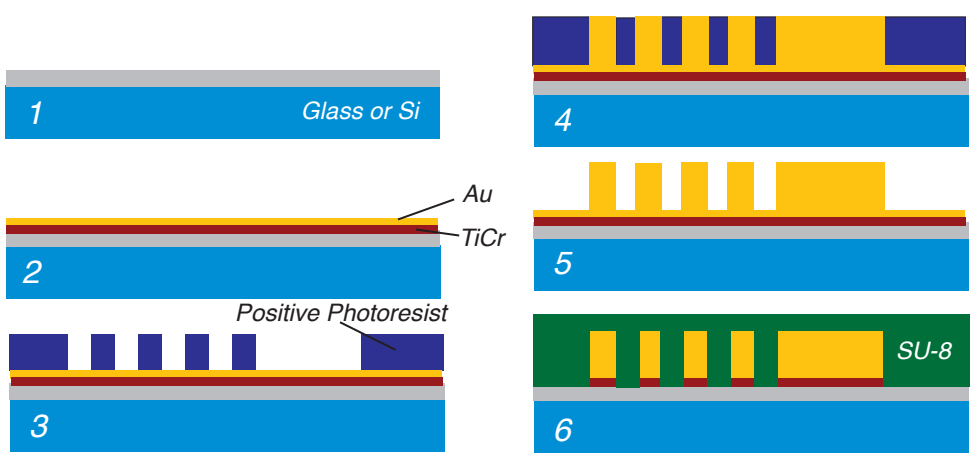

Figure 3.13: Step-by-step fabrication guide to glass substrate based microcoil fabrication. 


\subsubsection{Upisel-N Etching}

A more recent process, relied on pre-fabricated polyimide laminate sheets of $50 \mu \mathrm{m}$ thickness and a electronically deposited single sided copper coating of $18 \mu \mathrm{m}$ (Upisel-N, UBE Industries, Ltd.). The laminate was cleaned with acetone, and spin coated with a layer of AZ 1500 series positive photoresist (on the $\mathrm{Cu}$ side). Departing from the direct writer, a pre-made transparency mask (from AutoCAD drawings) was used for patterning. A Karl Süss MJB3 mask aligner was used for consistency, but ultimately was not needed as there is only one alignment step. After photoresist development, the excess $\mathrm{Cu}$ was removed by etching in $\mathrm{H}_{2} \mathrm{SO}_{4}: \mathrm{H}_{2} \mathrm{O}_{2}: \mathrm{H}_{2} \mathrm{O}$ in a ratio of $1: 2.3: 20$, respectively. The bridge connection was done by either soldering or gold wire boding, although the latter is difficult given that the base material is copper. To protect against oxidation a thin layer of MEMS wax was applied using a heat plate. The entire process is recorded in Figure 3.14.
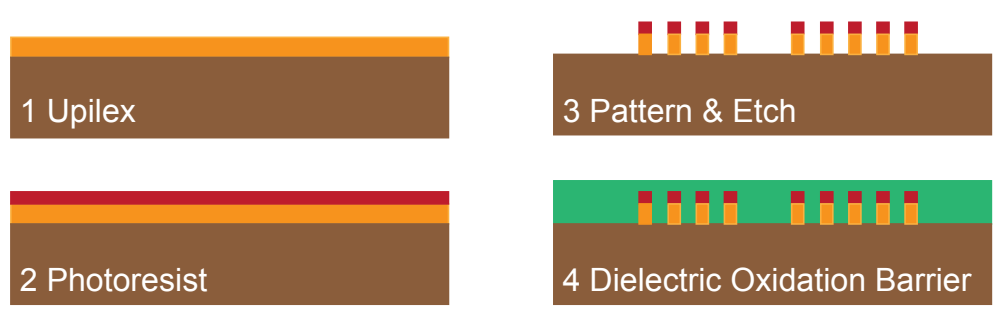

Figure 3.14: Complete fabrication guide to flexible copper/polyimide fabrication.

Micrographs of coils fabricated by both processes are shown in Figure 3.15 (Tunable and non-tunable coils are shown for the Upilex method). 

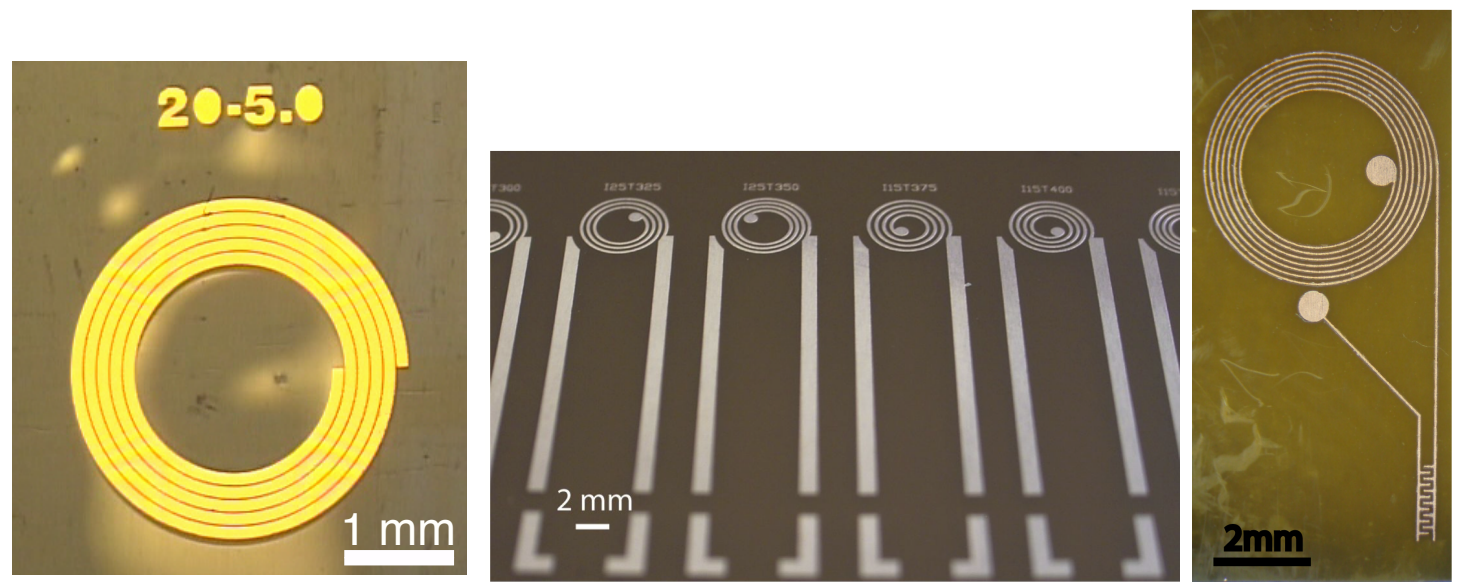

FIGURE 3.15: Planar micro fabricated resonators. Left: self resonant coil using the stray capacitance between turns for tuning. Middle: $\mathrm{Cu}$ coils fabricated on Upilex pre-plated polyimide foil for tunable coil. Right: Upilex polyimide fixed capacitor coil.

\subsection{Microcoil Characterization}

Prior to spectroscopic experimentation, planar microcoils were characterized in two ways; first by acquisition of self resonance by inductive sniffer coils and then by microimaging of field intensities around the microresonator. Importantly, previous issues of self resonance mis-detection were resolved by introduction of inductive measurement of the microresonators through the network analyzer.

\subsubsection{Network Analyzer}

Given the microresonator is inductively coupled to the pickup coil inside the probe, it's logical that they should be measured in a similar fashion. Accordingly, a simple sniffer coil was assembled and several planar microcoil resonate values were recorded by peak extraction from the reflection curve, see Figure 3.16. Self resonance values are easily be collected using this method; however, Q-Factor ${ }^{7}$ determination is more difficult since

\footnotetext{
${ }^{7}$ Quality factor is a dimensionless parameter describing resonator under-damping.
} 
the definition, $Q=f_{c} / \Delta f$, requires a bandwidth measurement at $-3 \mathrm{~dB}$ from the peak of the transmission curve. When using the reflectance, this value is interpolated and subject to mischaracterization. Therefore a new inductive measurement technique was pursued based on the transmission curve.
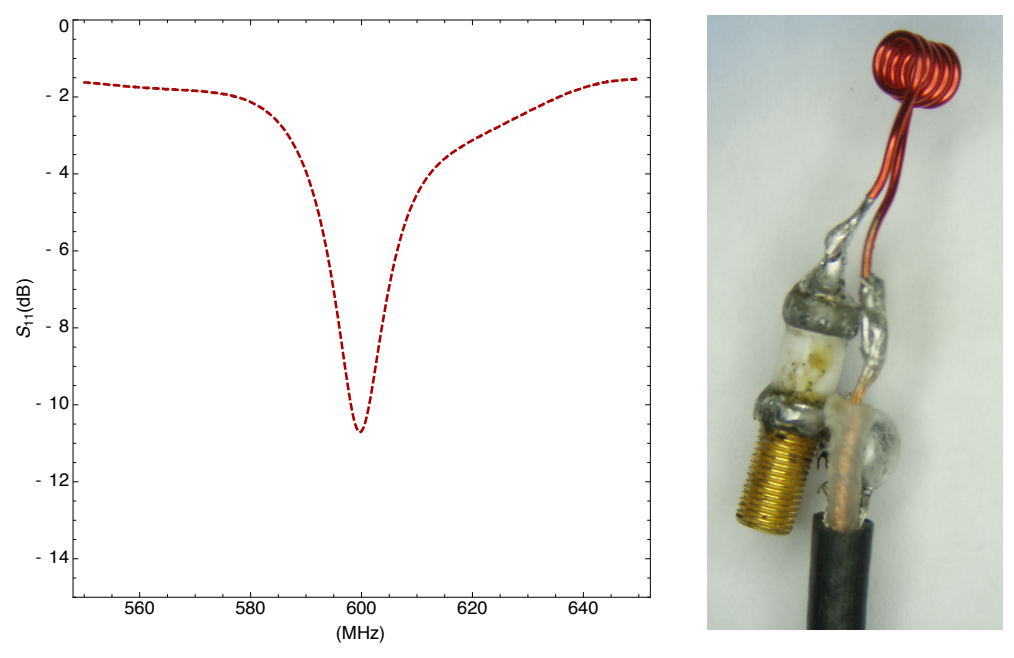

Figure 3.16: Left: Microresonator measured reflection using the sniffer coil. Right: Sniffer coil composed of simple wire solenoid and tunable capacitor soldered to a coax cable.

In a second approach, the electrical response of the resonators was tested using two circular loops lithographically etched onto a printed circuit board. The arrangement of the coils is such that their mutual inductance largely cancels. By introducing a tuned resonator into the proximity of the overlap region, the cancellation curve is replaced with a transmission pattern (see Figure 3.17). The resonance was then be observed by measuring the transmission loss $\left(S_{12}\right)$ through the double loop device with HP $8735 \mathrm{D}$ vector network analyzer. While the position of the resonance peak directly indicated the resonance frequency, the quality factor was determined from the width of the peak at $-3 \mathrm{~dB}$ from the maximum as $Q=\Delta \omega_{-3 d B} / \omega_{0}$.

Complete network analyzer data processing routines can be found in Appenidx D. 

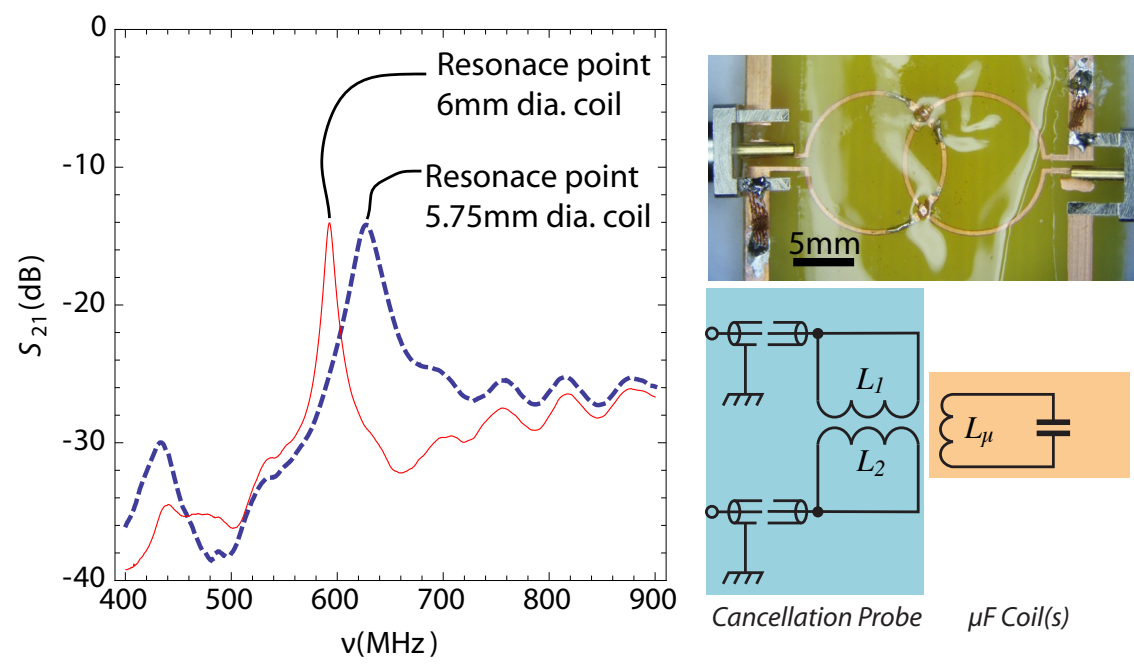

FiguRE 3.17: Left: Cancellation curve for two different coil geometries. The Q-factor is easily retrieved from this graph. Right: Cancellation device (top) and attached two coax cables. Equivalent circuit diagram (bottom) shows this system behaves similarly to the NMR probe.

\subsubsection{Microimaging}

While the cancelation loop can provide accurate resonances and Q-factors, it's important to know the distribution of the field around the microcoil. To image the field intensity around the microcoil, the NMR imaging mode is employed. The resonator chips are aligned inside a $12 \mathrm{~mm}$ NMR sample tube filled with doped water (i.e. $0.01 \mathrm{M} \mathrm{CuSO}_{4}$ and $0.5 \mathrm{M} \mathrm{NaCl}$ ). Magnetic resonance images are acquired in a perpendicular orientation in relation to the coil surface. Figure 3.18 shows a 256 X 256 pixel image demonstrating the signal intensity gain in water around the coil.

\subsection{NMR Experiments}

NMR experiments were conducted using a Varian VNMR spectrometer, operating at a static field strength of $14.1 \mathrm{~T}$. Images have been acquired with a Doty $12 \mathrm{~mm}$ micro 

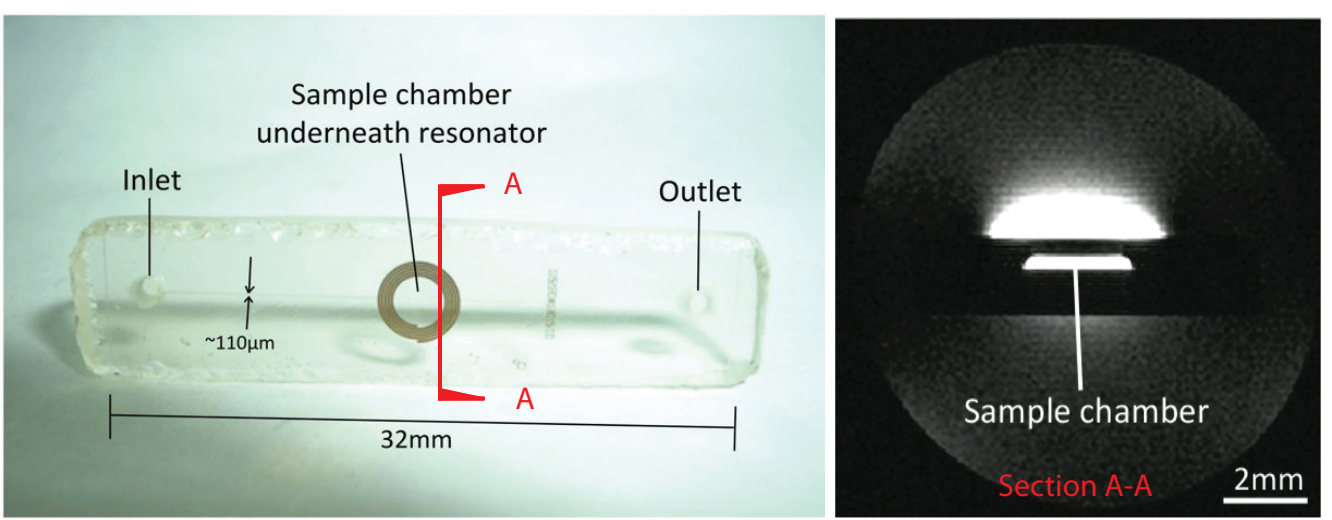

FiguRE 3.18: Left: Microfluidic device with integrated microresonator. Right: MRI image of microcoil signal intensity, shown spilling into the sample chamber of the microfluidic device [80].

imaging probe using a sample of $150 \mathrm{mM} \mathrm{NaCl}$ and $10 \mathrm{mM} \mathrm{CuSO}_{4}$ in $\mathrm{H}_{2} \mathrm{O}$. Gradient echo images were obtained using a Gaussian excitation pulse profile with 2 ms duration. Slice thickness was $0.5 \mathrm{~mm}$; a gradient echo time of $2 \mathrm{~ms}$ and a relaxation delay of $500 \mathrm{~ms}$ were used. The latter ensured that the image contrast was not affected by $\mathrm{T}_{1}$ relaxation. A dedicated NMR probe head was built for inductive coupling of microfluidic chips. The commonplace pneumatic sample transport mechanism was modified to accommodate rectangular chips instead of conventional $5 \mathrm{~mm}$ NMR sample tubes. The design of this probe is shown in Figure 4.11. The probe head structure shown in the figure was manufactured directly from the CAD drawings using a uPrint SE 3D fused deposition modeling system (Stratasys, Inc., Eden Prairie, MN), from acrylonitrile-butadienestyrene (ABS) copolymer. The primary coil structure was cut from self-adhesive copper tape (3M), and glued to two microscope slides that snapped into the sample holder on either side of the pillars shown in Figure 4.11. All spectra and images were processed using our own routines written in the Mathematica language (see Appendix E). 

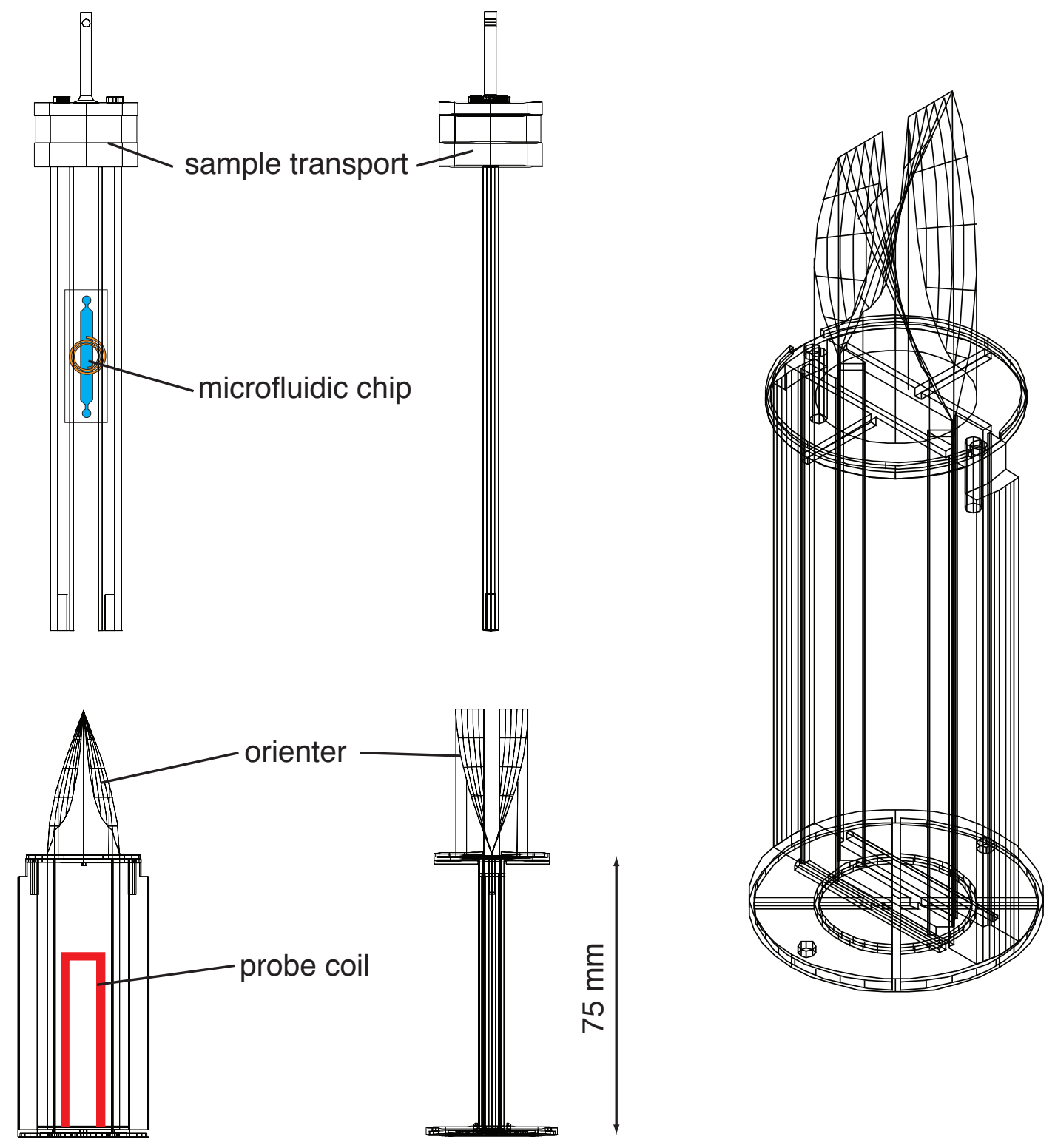

Figure 3.19: Schematic of the probe assembly from 3D CAD drawings of the sample orienter, sample deliver, and probe coil structure. 


\section{Chapter 4}

\section{Characterization and}

\section{Performance of Optimized Planar}

\section{Microcoils}

\subsection{Microcoil Characterization}

Multiple microcoil iterations were produced, with varying geometry, before optimum sensitivity and resolution was achieved. One obstacle encountered during the resonance frequency characterization using the cancellation loop was the difference in resonance noted going from bench top network analyzer to the NMR experiment environment. Depending on susceptibility differences in substrate type and adhesion method (resonator to microfluidic chip), the resonance would vary up to $100 \mathrm{MHz}$ from its original value. 
This shift was predictable however, and was addressed by biasing microcoils to a higher frequency. Results from the cancellation loop and microimaging are below.

\subsubsection{Resonance Frequency and Q Factor}
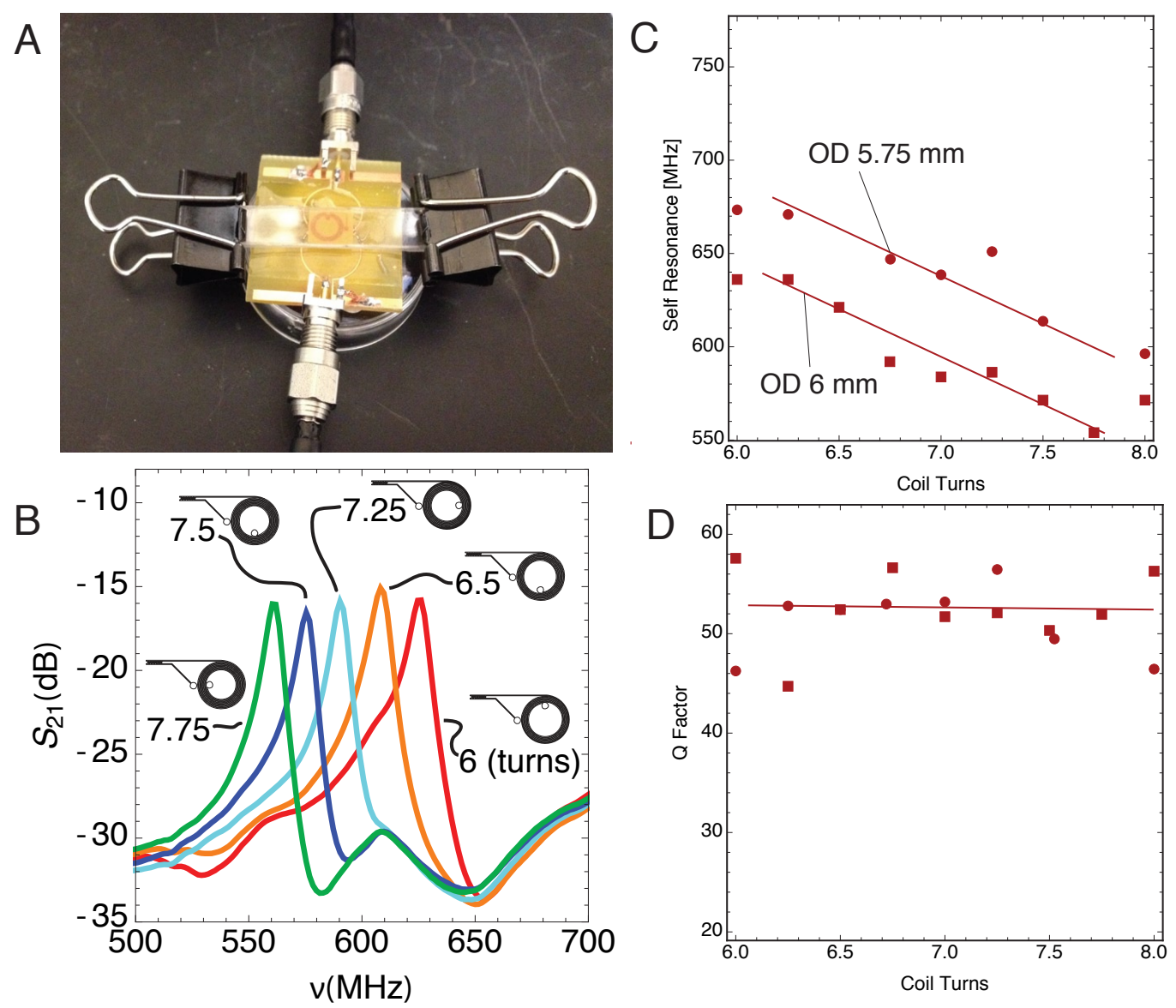

Figure 4.1: (a) Cancelation coil measurement technique using two clamped glass places to sandwich the microresonator. (b) Cancellation curve corresponding to different coil geometries, from which the resonance and Q-Factor are determined. (c) Resonance frequency and (d) quality factor of planar coil resonators as a function of number of turns. Results for coils of $5.75 \mathrm{~mm}$ and $6.00 \mathrm{~mm}$ outer diameter are shown.

The resonance frequencies and $Q$ factors obtained from the two sets of coils are shown in Figure 4.1. The coils were fabricated by etching Copper coated polyimide sheets, as discussed in chapter 3.5.2.2. The width of the conductor path was left constant at 
$100 \mu \mathrm{m}$, with a turn gap of $50 \mu \mathrm{m}$ for all cases. The number of turns was adjusted by $1 / 4$ turn between 6 and 8 total turns. The out-out diameter of the coils was $5.75 \mathrm{~mm}$ and $6 \mathrm{~mm}$. Demonstrated in Figure 4.1C, the resonance frequency drops with increasing number of turns and coil diameter, as expected. By contrast, the $Q$ factors seem largely constant with a medium value of 53 over the range of geometries described above. The large scatter observed in Figure 4.1D is caused by both measurement apparatus error (see Figure 4.1A) and fabrication inconsistency.

To minimize the error associated with measuring the microcoils using the cancellation loop, two glass plates were used to sandwich the coil to eliminated orientation effects on the cancellation curve, see Figure 4.1A. Clamping the coils with glass also ensured that the dielectric constant of the surrounding medium was the same each time. Collectively, the sandwiched resonator was consistently placed on the surface of the cancellation loop so that the copper clad side faced down and it maintained the same orientation of the leads.

Fabrication error was traced to one of three sources: (i) over etching of the sample (shown in Figure 4.2), (ii) underetching and poorly defined photoresist boundaries, and (iii) post fabrication surface treatment, typically using MEMs wax. The former two were often related since badly defined photoresist patterns would at first show signs of tract jumping and to compensate would be etched further, sometimes resulting in loss of geometry by over etching. 


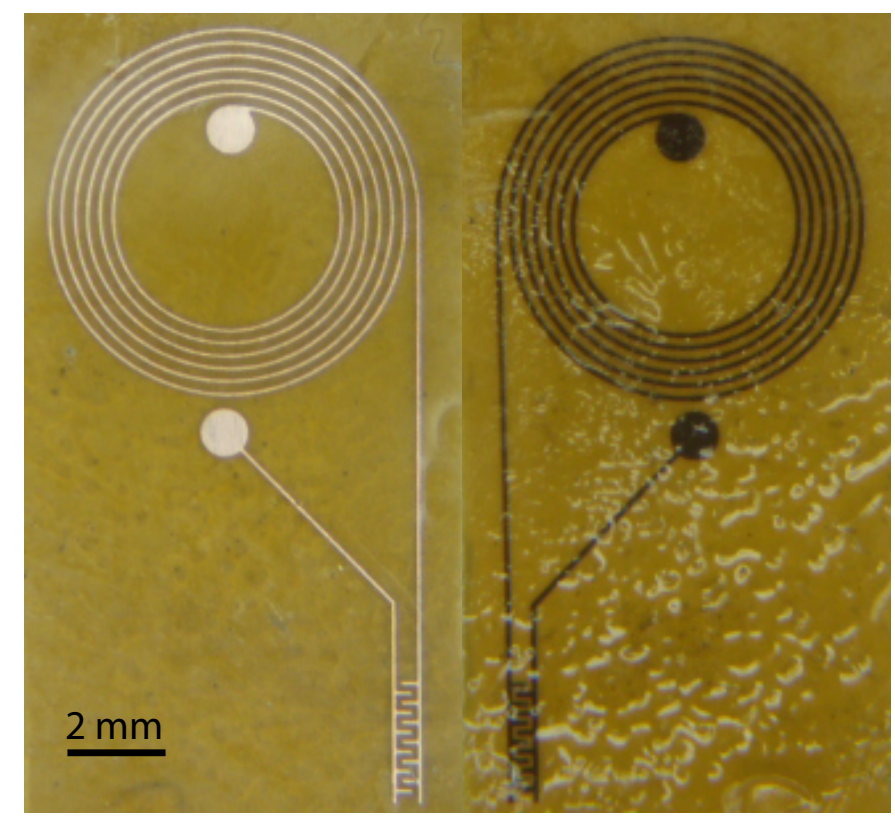

FIGURE 4.2: Left: Over etching of the original pattern was a significant source of measurement error. It can be seen in the above picture that the tracks of the copper are thinner than the inter-tract spacing. Right: Backside view of a microresonator with unequal application of MEMs wax producing light reflecting distortion patterns.

\subsubsection{Signal Strength Dependence on Applied Power}

In order to test the behavior of planar coil resonators in a magnetic resonance context, a coil was placed vertically in a $12 \mathrm{~mm}$ sample tube filled with doped water. Figure 4.3 shows gradient echo images obtained from this system with different excitation pulse intensities. The local enhancement of the radio frequency field due to the resonance of the coil is clearly visible in the images. The contrast stems from two sources: the current in the resonator increases the local RF field strength, leading to a larger pulse excitation angle. In addition, the resonant coupling of the microcoil to the probe causes the signal around the microcoil to be recorded at a higher sensitivity.

Figure 4.4 shows the comparison of signal intensity from the area close to the resonator versus background signal far away relative to the resonator. The maximum signal close 

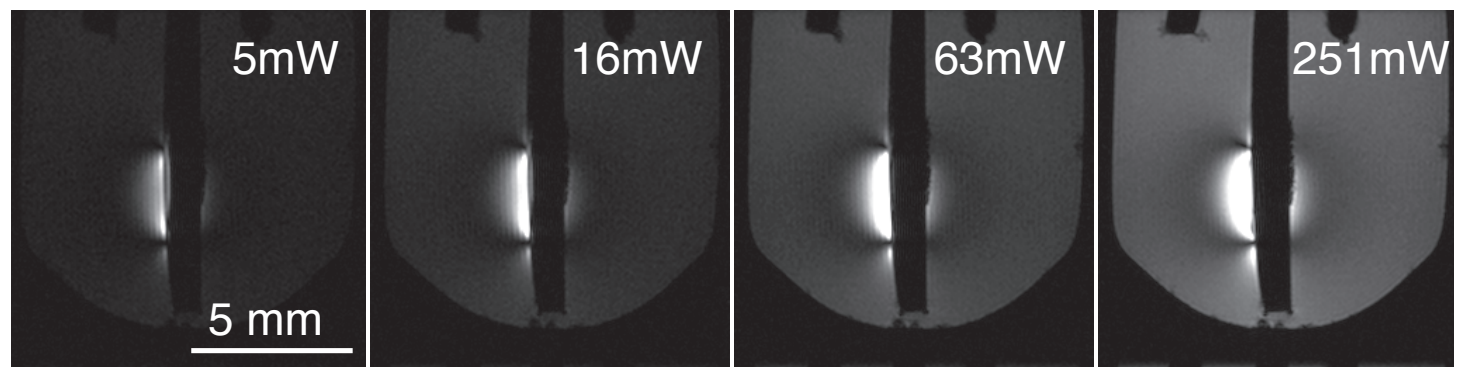

Figure 4.3: Gradient echo images of a planar resonator immersed in doped water at increasing excitation power.

to the resonator is a factor of 3.5 higher than in the background, and it is reached at a power level of $23 \mathrm{dBm}$, whereas the background signal peaks at a higher power level of $33 \mathrm{dBm}$. Since the $B_{1}$ field strength is proportional to the square root of the applied power, this corresponds to a factor of 3.16. The close agreement is verification of the correspondence principle, which that relates the NMR experimental sensitivity to the probe efficiency (magnetic field strength generated per square root of power).

In these experiments, the resonator coil was coupled inductively to the tuned $\mathrm{Litz}^{1}$ coil built into the Doty micro imaging probe. As described in Chapter 3.4.1, when both the resonator and the probe are tuned to the same frequency, the coupling between them causes a splitting of the resonance. The resonance frequency of the planar microcoil has to be chosen such that one of the two lines coincides with the Larmor frequency (600 MHz). With proper matching of the probe, the available power is then split evenly between the probe coil and the resonator coil. In the case of microimaging experiments using the factory made Litz coil, the coupling splits the resonance by $\approx 40 \mathrm{MHz}$ (i.e. resonances at 580 and $620 \mathrm{MHz}$ ). Unfortunately, the tuning range of the micro imaging probe is only $20 \mathrm{MHz}$, making either resonance inaccessible. Therefore, the experiment

\footnotetext{
${ }^{1}$ Litz (woven) coil utilizes etched foil patterns with insulated crossovers to obtain RF flux transparency and improved SNR [99].
} 


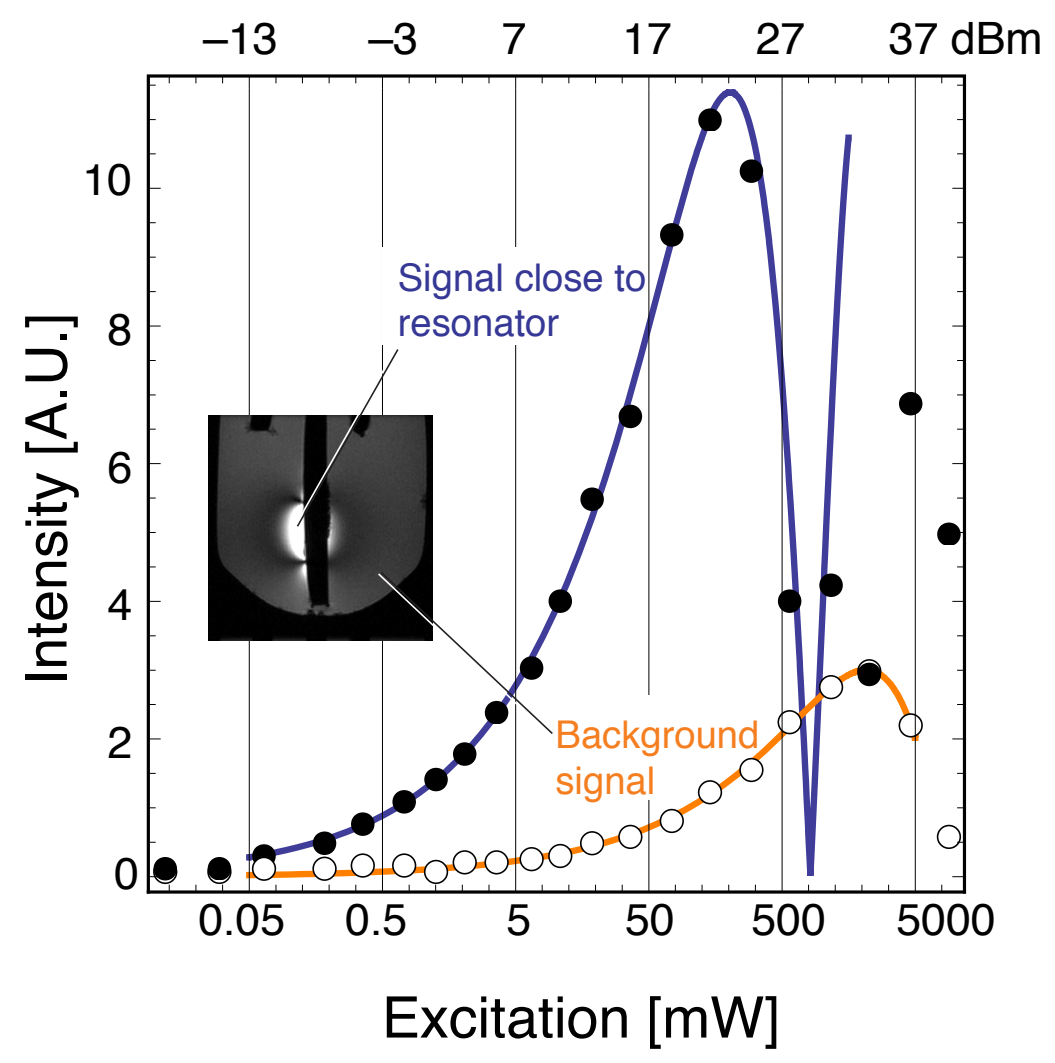

FIGURE 4.4: Dependence of signal strength on excitation power.

was carried out with a resonator at lower self resonance to offset one of the lines to fall at $600 \mathrm{MHz}$. This results in inefficient coupling, since the majority of the available power is dissipated in the probe pickup coil instead of the microresonator. Thus, the reported magnification factor of 3.16 is not representative of the true maximum achievable probe efficiency. Nonetheless, this experiment is only meant to validate the correspondence principle, and provide information on the shape of the RF field around the microresonator. 


\subsection{Lineshape and Signal/Noise Ratio}

A quantitative assessment of the probe efficiency and sensitivity was acquired from a tuned $600 \mathrm{MHz} \mathrm{Cu}$ /polyimide coil attached to a microfluidic PMMA chip with a circular sample chamber of $5 \mathrm{~mm}$ diameter and $200 \mu \mathrm{m}$ depth. The collectively packaged coil/microfluidic device was inserted into the probe assembly shown in Figure 4.11. As explained in Chapter 3.4.1, the probe coil was not tuned to $600 \mathrm{MHz}$ to avoid resonance splitting.

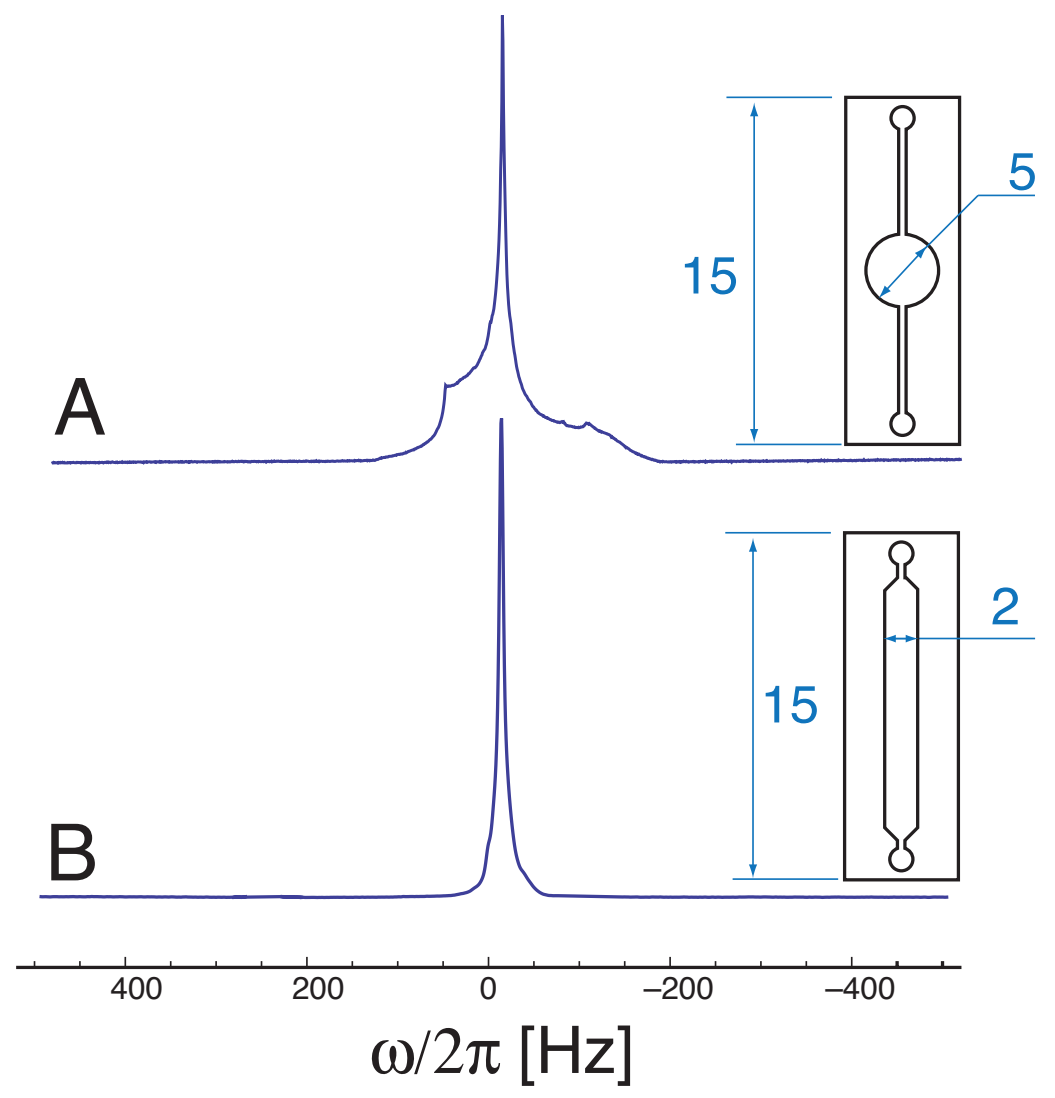

FIGURE 4.5: PMMA based microfluidic devices with (A) rectangular cross section and (B) circular cross section. Chips are $0.6 \mathrm{~mm}$ in thickness with $200 \mu \mathrm{m}$ thick sample chamber. 
Figure $4.5 \mathrm{~A}$ shows the spectrum of a sample of $5 \% \mathrm{H}_{2} \mathrm{O}$ in $\mathrm{D}_{2} \mathrm{O}$ obtained from this setup. A single transient was acquired after a $90^{\circ}$ pulse. The magnetic field was optimized with the spectrometer shim to be as homogeneous over the sample region as possible, corresponding to a improved lineshape/peak height. A shim is a device used to adjust the homogeneity of a magnetic field. In an ideal case, we would expect to see vertical lines representing peaks much like a delta function. As Figure 4.5 clearly shows though, there is substantial inhomogeneous broadening of the resonance line. This can be attributed to susceptibility mismatch between the chip material (PMMA) and the aqueous sample. Modifying shim settings reduced the overall width of the line by a factor of 2 over what is shown in Figure 4.5A, but at the expense of the sharpness/height of the central peak. The sensitivity was determined from this data by determining the SNR in the time domain (see figure 4.6), defined as the maximum signal height in the FID divided by twice the root mean square noise amplitude [1]:

$$
\mathrm{SNR}_{t}=1406 \pm 5
$$

The susceptibility mismatch between the sample and the chip material leads to a marked dependence of the resonance lineshape on the shape of the sample compartment. Illustrated in Figure 4.5 are two PMMA based fluidic sample chamber arrangements. As mentioned earlier, susceptibility mismatch is to blame for the artifacts seen in the foot of Figure 4.5A (the round chamber). The same problem also applies to the rectangular case; however, due to the orientation of the chamber walls relative to the magnetic static field, the effect can largely be shimmed away using lower order shims (e.g. Z, Y, X, Z ${ }^{2}$, 
$\mathrm{XY}, \mathrm{YZ} \cdots$ etc.). For this reason, the rectangular linear chip configuration was selected for NMR spectroscopy experiments.

A

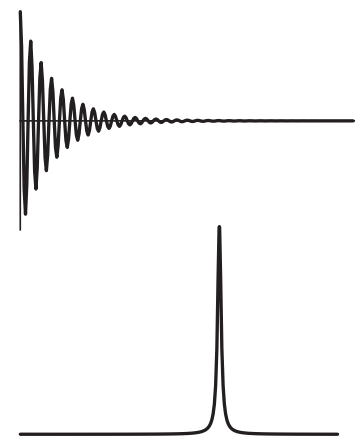

C

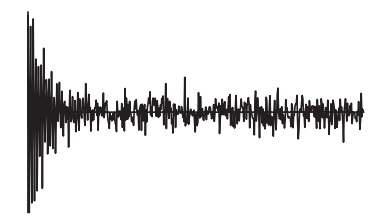

D

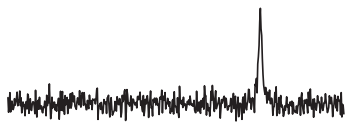

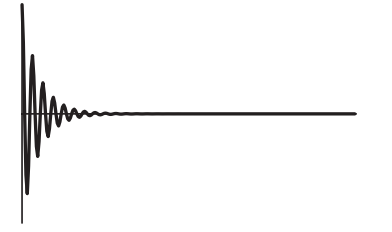

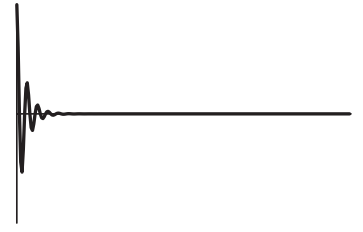

time $\longrightarrow$
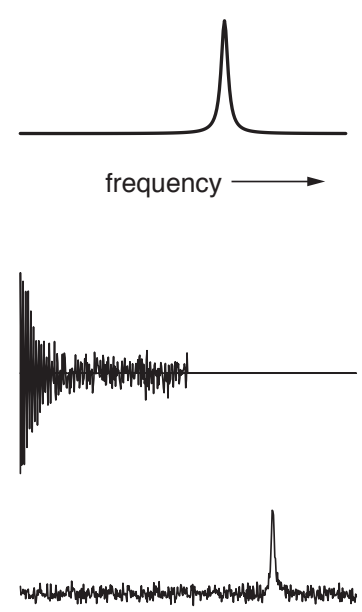
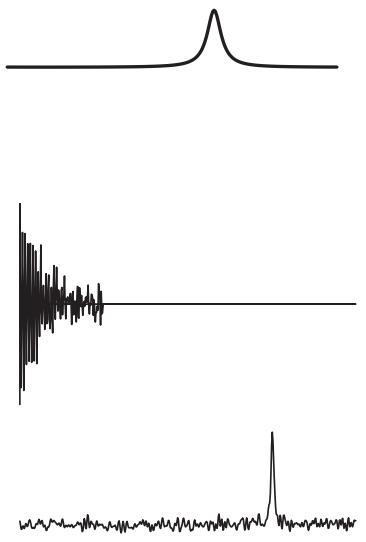

FiguRE 4.6: (A) and (B) are the free induction decay and Fourier transformed spectrum, respectively, for an idealized case. The progression from left to right show that the more rapidly the FID decays the broader the line in the corresponding spectrum. (C) and (D) show that for a quickly decaying signal the noise carries on unabated for the during to the experiment. By taking only the part of the FID containing the signal the SNR can be improved [41].

\subsection{Limit of Detection}

The single-scan limit of detection, Equation 3.3 reprinted below for reference, was determined to be $\mathrm{LOD}_{t}=2.7 \mathrm{nmol} \cdot \mathrm{s}^{1 / 2}$.

$$
\mathrm{LOD}_{t}=\frac{N_{S}}{\mathrm{SNR}_{t} \sqrt{\Delta f}}
$$


It was assumed that the bandwidth in which the spins resonate was $\Delta f=200 \mathrm{~Hz}$, which comes from the water peak total linewidth. Bart et al. [11] reported a value of 0.47 $\mathrm{nmol} \cdot \mathrm{s}^{1 / 2}$ for their micro stripline probe, obtained from a sample of pure isopropanol, also operating at $600 \mathrm{MHz}$. In order to compare these numbers, their value has to be multiplied by two since it was obtained using four scans rather than one. Secondly, their sample volume was $600 \mathrm{nl}$, compared with $1.2 \mu \mathrm{l}$ in our case. The mass sensitivity increases with the inverse cube root of the coil volume [19]. Scaling our $\mathrm{LOD}_{t}$ to $600 \mathrm{nl}$ (a factor of 1.25) and 4 scans (a factor of 2), the value is determined to be $0.38 \mathrm{nmol} \cdot \mathrm{s}^{1 / 2}$. We can perform this same normalization process on other reported coils (summarized in Figure 2.14). With the exception of Gruschke et al. and Renaud et al. who reported sensitivities corresponding to nLOD values of 7730 and $92000 \mathrm{nmoll} \cdot \mathrm{s}^{1 / 2}$, respectively, our reported value matches up well with the field $[14,64,100]$.

Figure 4.7 shows a nutation spectrum obtained from a glass rectangular sample chamber chip at an excitation power $(P)$ of $12.6 \mathrm{~W}$. From this experiment the probe efficiency can be calculated from the equation:

$$
\eta_{\text {probe }}=\frac{B_{1}}{\sqrt{P}}=\frac{1}{4 \tau_{90} \sqrt{P}}
$$

The $90^{\circ}$ pulse length $\left(\tau_{90}\right)$ is $9 \mu \mathrm{s}$, which corresponds to a probe efficiency of $0.184 \mathrm{mT} /$ $\sqrt{W}$, or (for protons) $7.83 \mathrm{kHz} / \sqrt{W}$. It should be noted that this is the efficiency of the entire transmission pathway, including losses in the cables, preamplifier setup, filters, and the probe. The $A_{450} / A_{90}$ ratio is $71 \%$, and $A_{810} / A_{90}$ is $36 \%$. Compared with a standard $5 \mathrm{~mm}$ liquid-state probe the radio frequency homogeneity doesn't measure up 


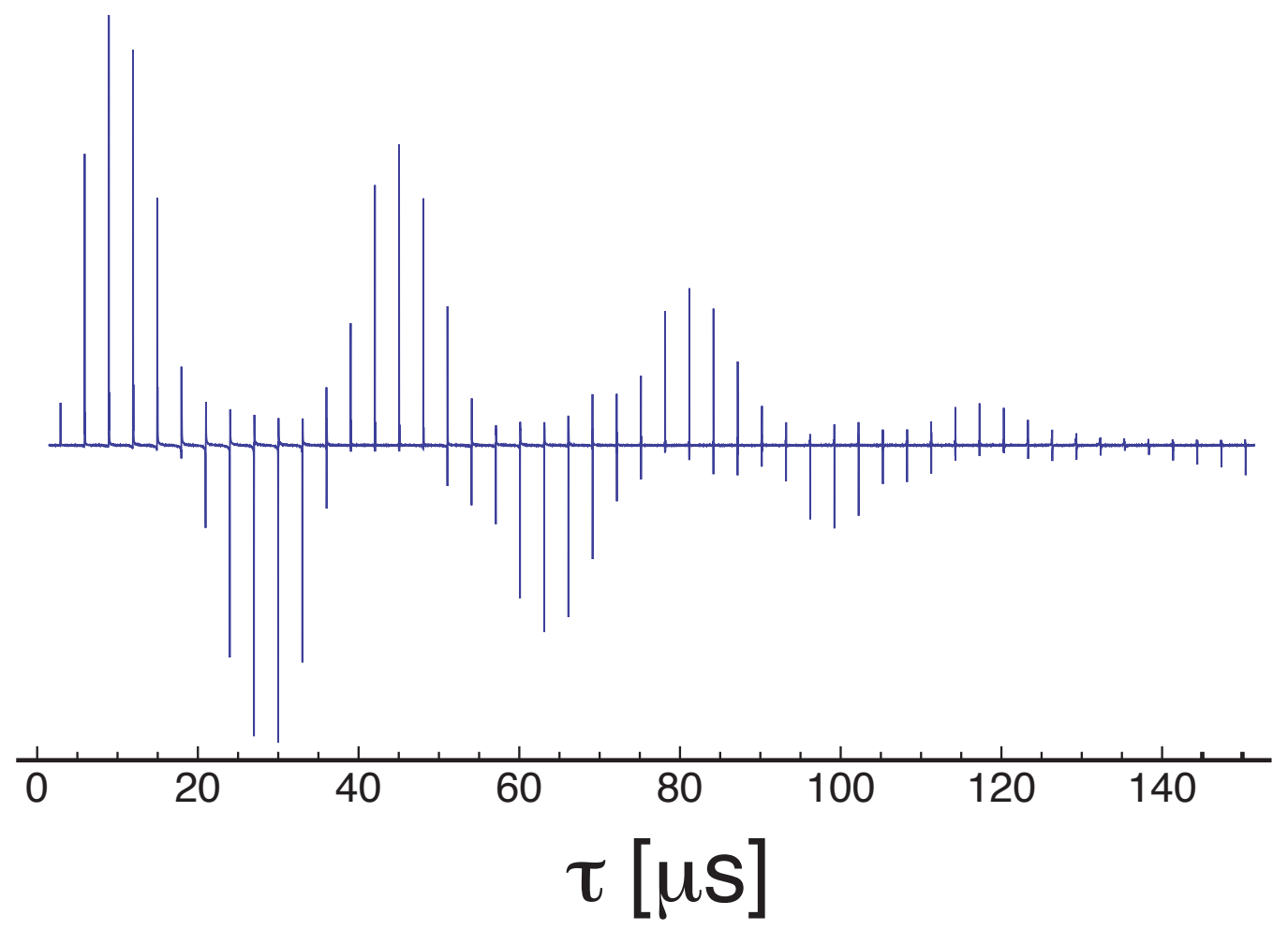

Figure 4.7: Nutation spectrum of a sample of $5 \% \mathrm{H}_{2} \mathrm{O}$ in $\mathrm{D}_{2} \mathrm{O}$.

very well in the described system. However, it does compare favorably with what other groups using planar micro coils have reported.

\subsection{Experimental Results}

As described in Chapter 4.2, a rectangular chip (see Figures 4.8A and 4.5) was used in all experiments. The chip was manufactured from borofloat glass, as described in Chapter 3.5.1.1. The sample chamber consists of a long linear channel of $2 \mathrm{~mm}$ width, $150 \mu \mathrm{m}$ depth, and $12 \mathrm{~mm}$ length. This chamber was combined with a $\mathrm{Cu} /$ polyimide resonator of 6.75 turns and $6 \mathrm{~mm}$ outer diameter. The active sample volume is approximately $2 \times 4$ $\times 0.15 \mathrm{~mm}^{3}=1.2 \mu \mathrm{l}$, giving the total number of spins in the system as $1.6 \times 10^{15}$. Figure 


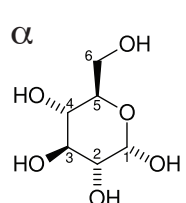

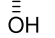

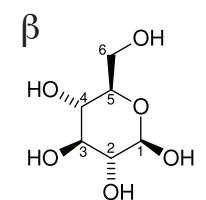

OेH

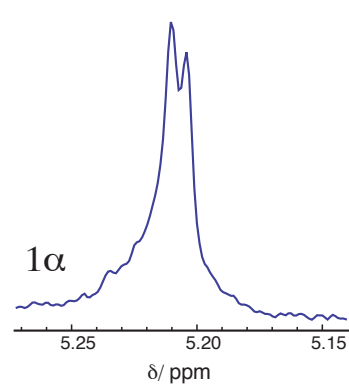

$D$

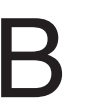

A
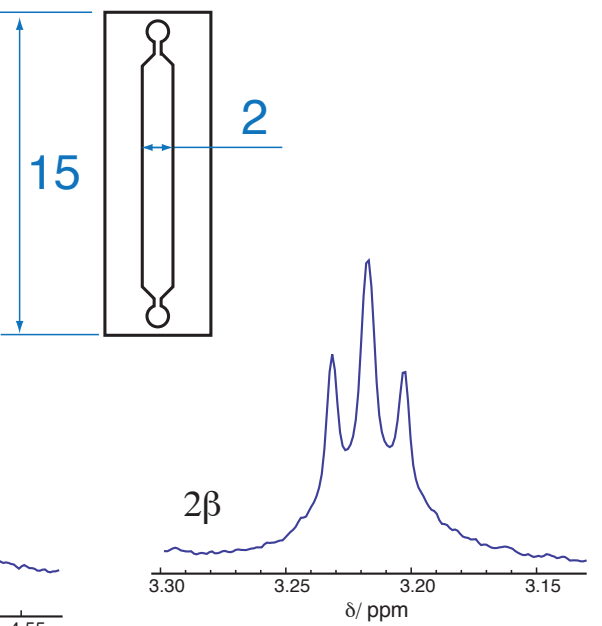

; $\delta / \mathrm{ppm}$
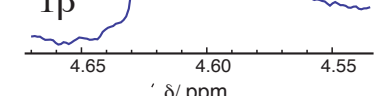

'

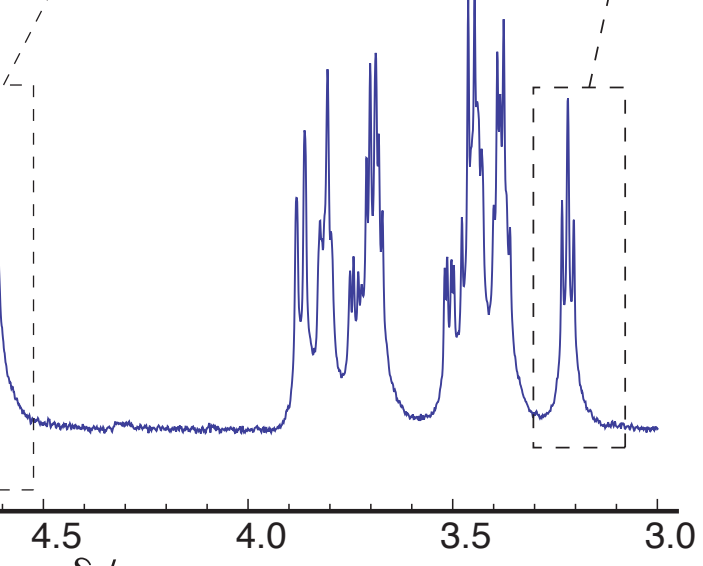

$\delta / p p m$

Figure 4.8: ${ }^{1} \mathrm{H}$ spectrum of glucose obtained from $1.2 \mu \mathrm{l}$ of sample in an inductivelycoupled microfluidic chip. Spectral resolution is about $4.5 \mathrm{~Hz}$.

4.8B shows a single scan spectrum of a sample of $500 \mathrm{mM} \alpha$-D-glucose in $\mathrm{D}_{2} \mathrm{O}$. The water signal was suppressed by $1 \mathrm{~s}$ pre-saturation at $0.25 \mathrm{~mW}$ at a frequency corresponding to $\delta=4.76$ ppm. 8000 complex data points were acquired after a $90^{\circ}$ pulse over a spectral width of $10 \mathrm{kHz}$, and processed with Gaussian line broadening of $1.5 \mathrm{~Hz}$.

The susceptibility broadening is minimized in the case of the rectangular sample chamber, and the remaining inhomogeneity was mostly be removed by shimming. The inherent line width (FWHM) was determined by fitting the triplet line with a Lorentzian line 


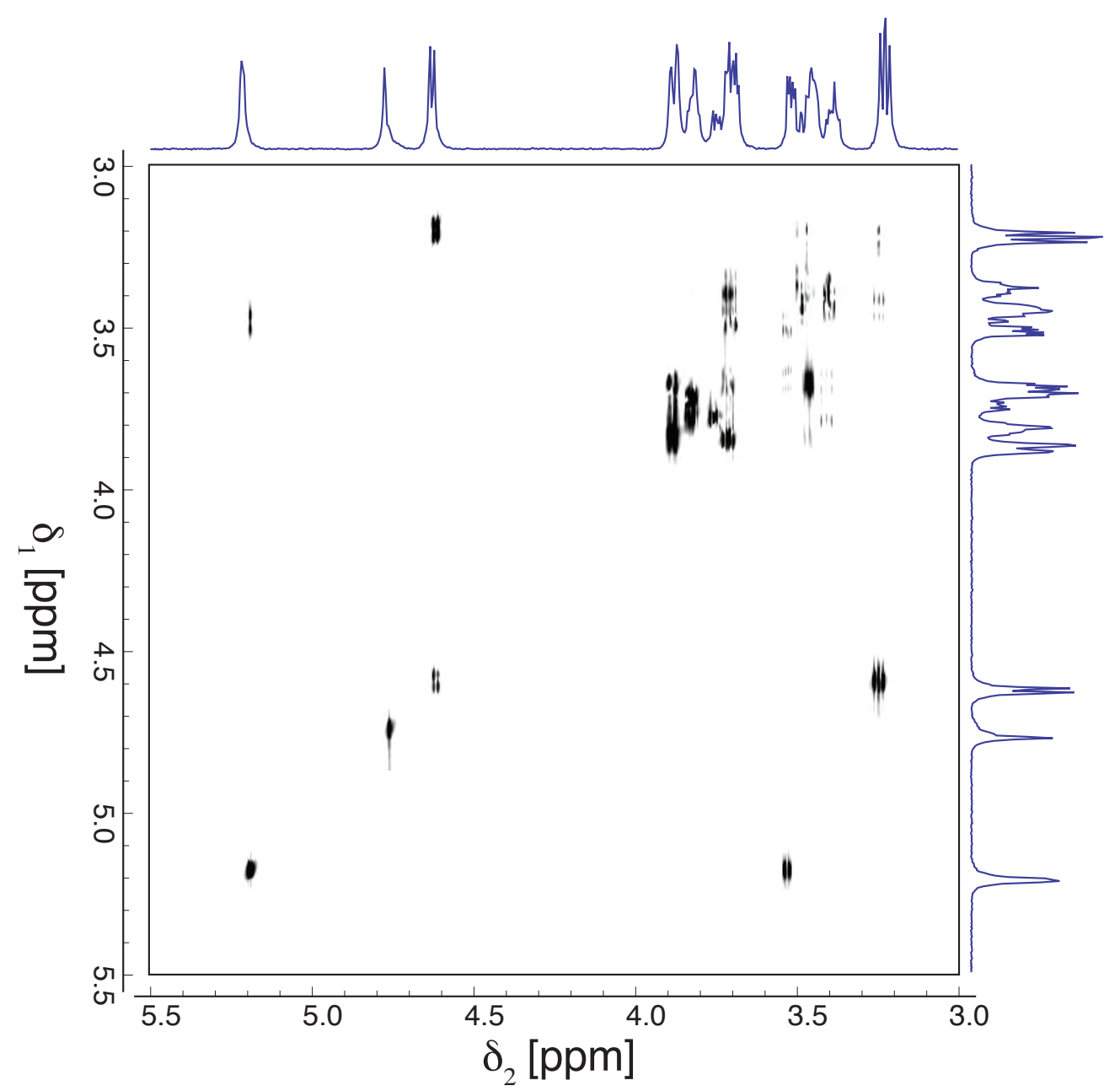

Figure 4.9: COSY spectrum of $1.2 \mu \mathrm{l} 500 \mathrm{mM}$ glucose in $\mathrm{D}_{2} \mathrm{O}$ with water suppression by pre-saturation. $256 t_{1}$ increments were acquired over a total experiment time of $4 \mathrm{~h}$. The data was extended to $512 t_{1}$ points by linear prediction with 64 coefficients.

shape of $4.5 \mathrm{~Hz}$ width. Literature custom dictates the sensitivity of micro-NMR systems be evaluated through the anomeric proton (position 1) of glucose. As is well known, glucose isomerizes into $\alpha$-D-glucopyranose and $\beta$-D-glucopyranose (shown top left in Figure 4.8) in aqueous solution over several hours at room temperature. Therefore, the sample was prepared a minimum of $24 \mathrm{~h}$ before the NMR measurements were made. The anomeric proton for $\alpha$ and $\beta$ isomers appear at $4.6 \mathrm{ppm}$ and $5.2 \mathrm{ppm}$, respectively [35]. 
The signal from the $\beta$ anomeric proton was used for sensitivity assessment. The singlescan SNR was recorded to be 106. With a assumed concentration of the $\beta$-D-glucopyranose of $250 \mathrm{mM}$, this corresponds to a specific SNR of $320 \mu \mathrm{mol}^{-1}$. For comparison, Massin et al. reported a value of $88 \mu \mathrm{mol}^{-1}$ for a conventionally coupled (wired) planar micro coil of very similar dimensions. Their experiment was carried out at a proton resonance frequency of $300 \mathrm{MHz}$, but the quoted value was scaled to $600 \mathrm{MHz}$, and so is directly comparable to our result. The mass sensitivity can be converted into a limit of detection, corresponding to the amount of sample that needs to be present to yield a SNR of 3 for an acquisition time of $1 \mathrm{~s}^{2}$. The normalized limit of detection, thus defined, is given by [15]:

$$
\mathrm{nLOD}_{m}=3 \frac{N_{S} \sqrt{t_{\mathrm{Exp}}}}{\mathrm{SNR}}
$$

where $t_{\operatorname{Exp}}$ denotes the time for the experiment. From the SNR reported above, we obtain $\mathrm{nLOD}_{m}=22.2 \mathrm{nmol} \cdot \mathrm{s}^{1 / 2}$, assuming an experiment time of $5.5 \mathrm{~s}$ (which includes the relaxation delay). Bart et al. have reported an almost identical value of $\mathrm{nLOD}_{m}=$ $22.3 \mathrm{nmol} \cdot \mathrm{s}^{1 / 2}$ for the sucrose anomeric proton in a volume of $0.6 \mu \mathrm{l}$ in a micro striplilne probe.

As demonstrated in Figure 4.9, it is possible to acquire homonuclear two-dimensional spectra using the same setup. This is of particular importance in the context of the analysis of the complex mixtures. Figure 4.9 shows a correlation spectrum (COSY) obtained from $1.2 \mu \mathrm{l}$ of $500 \mathrm{mM}$ glucose $\mathrm{D}_{2} \mathrm{O}$.

\footnotetext{
${ }^{2}$ The nLOD varies from the LOD in that reported SNR, sample volume, and total measurement time are collected and then scaled to $600 \mathrm{MHz}$.
} 


\subsection{Conclusions}

The original motivation for this project was to perform metabolomics on a chip and monitor non-invasively through NMR spectroscopy. The reported LOD in this thesis (e.g. $0.47 \mathrm{nmol} \cdot \mathrm{s}^{1 / 2}$ ) approaches the sensitivity required for interesting applications in metabolomics. This was a normalized/scaled value with the a sample size of $1.2 \mu \mathrm{l}$. In actual applications, this number would be expected to be tens of nanoliters [23], implying that the coil sizes need to scaled down further. One issue of doing this is thermal noise, which can be a dominate signal contribution for coils with diameters $\leq 1 \mathrm{~mm}$. A more pressing problem is resolution, which if not addressed in the fabrication process by susceptibility matching materials, can cause extreme linebroadening to the point where spectra are indistinguishable. Summarized in Chapter 4.6 are ongoing efforts towards resolving this limitation.

\subsection{Future Work}

Efforts moving forward will focus on two areas: microcoil/probe optimization and susceptibility mismatched materials. Reported resolution values from Olson et al. of $0.6 \mathrm{~Hz}$ suggest that there is still much improvement left in this area [23]. Indeed, one of primary reasons microNMR has not been adopted in earnest is because of resolution limitations. Preliminary computational results looking at susceptibility mismatched materials indicate that compensation structures could be placed in close proximity to boundaries, where mismatched materials meet, to homogenize the magnetic field. Figure 4.10 shows computational results of how this could work in practice. Sample chambers containing 


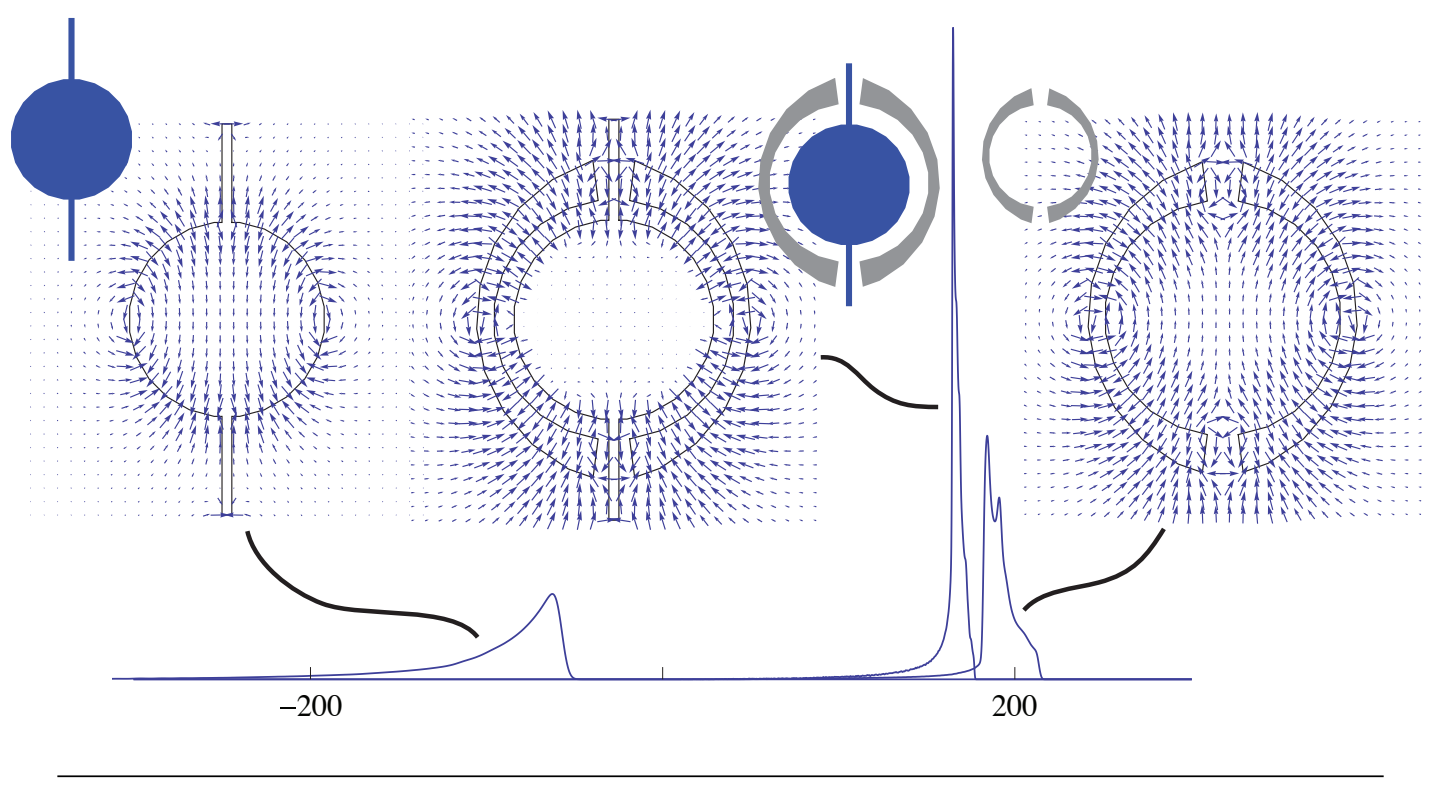

FIGURE 4.10: Preliminary computational results for susceptibility mismatched materials. In the top row magnetic field lines are approximated for three varying structures and their associated magnetic susceptibilities. The bottom row predicts what each

NMR line would look like based on the geometry and materials present.

solutions of different susceptibility than the chip material are surrounded with a compensating area of either diamagnetic or paramagnetic material, depending on the sign of the magnetic field at the boundaries.

The other area of focus going forward is in optimization of existing probe and microcoils. Shown in Figure 4.11 is the latest purposed layout of the probe coil. In this instance, the single induction loop is replaced with symmetric planar coils, made from copper foil, situated $\leq 3 \mathrm{~mm}$ from each chip face. The microfluidic device is tightly engulfed by the pickup coils, providing a better filling factor and improved sensitivity. A bonus advantage of this arrangement is a boost in resolution due to the high symmetry of the duel probe coils. 


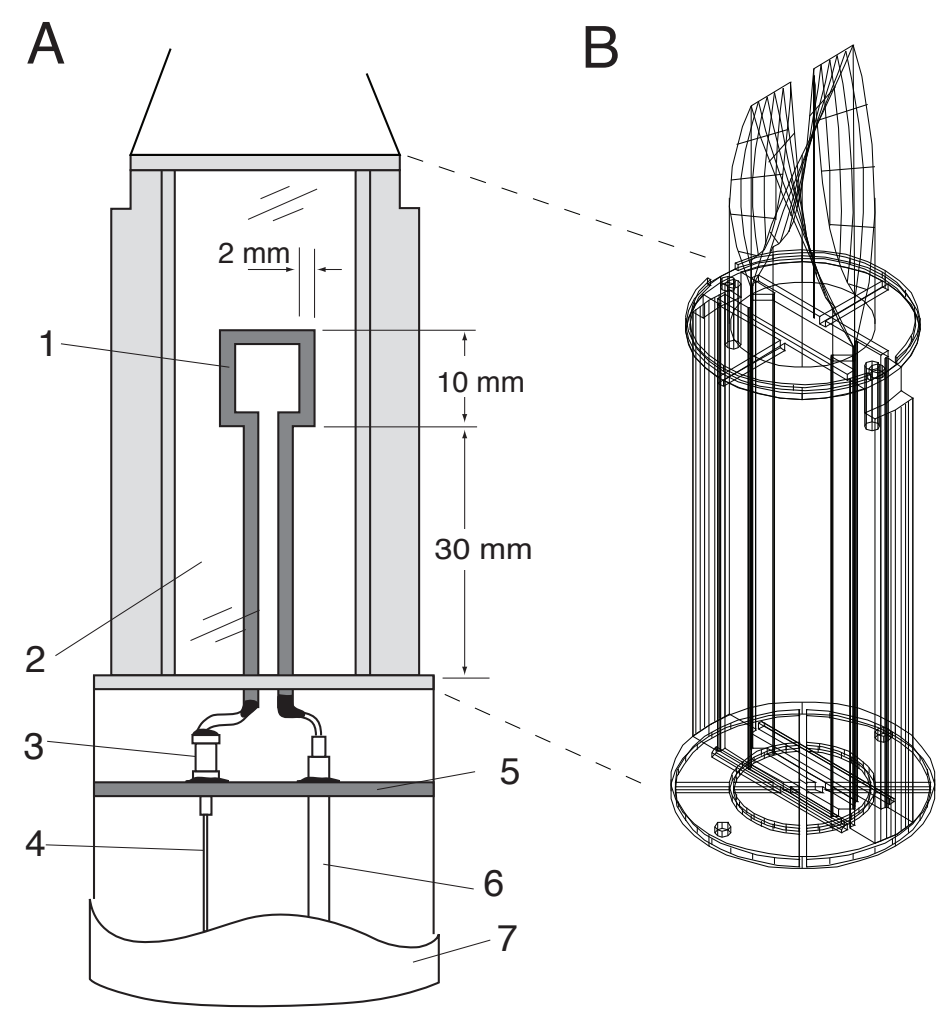

Figure 4.11: Schematic of the probe assembly (A) and 3D CAD drawing of the sample orientation / support structure (B). 1: Probe coil, made from self-adhesive $\mathrm{Cu}$ tape mounted on 2: support glass slide; 3: matching capacitor $(210 \mathrm{pF}) ; 4$ : matching adjustment rod; ground plane; 6: semirigid coaxial cable; 7: probe sheath. 


\section{Chapter 5}

\section{Final Remarks}

Microfluidic devices integrated with self-resonant planar coils can be coupled inductively to the spectrometer, allowing for high-resolution NMR spectroscopy with sensitivities similar to those obtained from other micro-NMR approaches. The biggest advantage of the inductive coupling mode is that it allows for easy exchange of the sample, since there is no tethered wire leads. While the demonstration experiments reported here have used very simple fluidic geometries, this opens the possibility to equip more complex lab-on-a-chip devices with planar resonators. The spectral resolution is limited by the susceptibility mismatch between the fluidic chip material and the sample. Currently, this requires avoidance of edges perpendicular to the magnetic field near the sample volume. This is a severe restriction in the design of NMR LOC devices. Efforts towards eliminating this constraint are currently underway by the introduction of compensation structures as outlined previously. 
Appendix A

\section{Inductive Coupling Calculation}




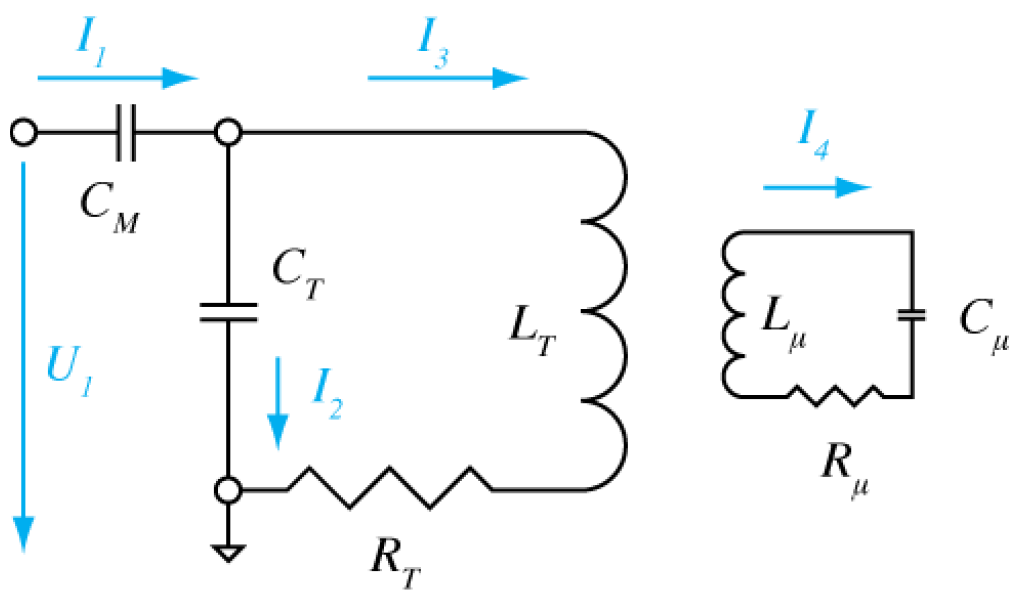

\section{Circuit equations and Ohm's law}

ProbeCircuit $=\{I 1==I 2+I 3$, ULT + URT - UCT $==0\}$

$\{\mathrm{I} 1=\mathrm{I} 2+\mathrm{I} 3,-\mathrm{UCT}+\mathrm{ULT}+\mathrm{URT}=\mathbf{0}\}$

Resonatorcircuit $=\{\mathrm{ULm}+\mathrm{URm}+\mathrm{UCm}=\mathbf{0}\}$

$\{\mathrm{UCm}+\mathrm{ULm}+\mathrm{URm}==0\}$

MatchingCircuit $=\{\mathrm{UCa}+\mathrm{UCT}-\mathrm{U} 1=0\}$

$\{-\mathrm{U} 1+\mathrm{UCa}+\mathrm{UCT}==0\}$

OhmsLaw $=\{$ ULT $==$ I $\omega$ LT I3 + I $\omega$ k Sqrt [LT Lm] I4, URT == RT I3, I $\omega$ CT UCT $==$ I2,

I $\omega \mathrm{Cm} \mathrm{UCm}=\mathrm{I} 4, \mathrm{ULm}=\mathrm{I} \omega \mathrm{Lm} \mathrm{I4}+\mathrm{I} \omega \mathrm{k}$ Sqrt $[\mathrm{LT} \mathrm{Lm}] \mathrm{I3}, \mathrm{URm}=\mathrm{Rm} \mathrm{I} 4, \mathrm{I} \omega \mathrm{Ca}$ UCa $==\mathrm{I} 1\}$

$\{\mathrm{ULT}==\dot{\mathbb{1}} \mathrm{I} 3 \mathrm{LT} \omega+\dot{\mathbb{1}} \mathrm{I} 4 \mathrm{k} \sqrt{\mathrm{LmLT}} \omega, \mathrm{URT}==\mathrm{I} 3 \mathrm{RT}, \dot{i} \mathrm{CT} \mathrm{UCT} \omega==\mathrm{I} 2$,

i $\mathrm{Cm} \mathrm{UCm} \omega==\mathrm{I} 4, \mathrm{ULm}==$ i I $4 \mathrm{Lm} \omega+$ i I $3 \mathrm{k} \sqrt{\mathrm{Lm} \mathrm{LT}} \omega, \mathrm{URm}==\mathrm{I} 4 \mathrm{Rm}$, i Ca UCa $\omega==\mathrm{I} 1\}$

\$Assumptions $=\mathrm{LT}>0 \& \& \mathrm{k}>0 \& \& \mathrm{Lm}>0 \& \& \mathrm{RT}>0 \& \& \mathrm{CT}>0 \& \& \mathrm{Cm}>0 \& \& \mathrm{Rm}>0 \& \& \mathrm{Ca}>0 \& \& \mathrm{I} 1>0 \& \& \omega>0$

$\mathrm{LT}>0 \& \& \mathrm{k}>0 \& \& \mathrm{Lm}>0 \& \& \mathrm{RT}>0 \& \& \mathrm{CT}>0 \& \& \mathrm{Cm}>0 \& \& \mathrm{Rm}>0 \& \& \mathrm{Ca}>0 \& \& \mathrm{I} 1>0 \& \& \omega>0$

FIGURE A.1: Inductive coupling calculation part (a). 


\section{Solutions}

These equations can be solved simultaneously for the currents $I_{3}$ and $I_{4}$, as well as the input voltage $U_{1}$. The input current $I_{1}$ is used as an input parameter. Together, that allows to compute the input impedance of the circuit.

solutions = Solve [Join [ProbeCircuit, ResonatorCircuit, MatchingCircuit, OhmsLaw],

$\{$ I3, I4, UCT, ULT, U1, ULm, URm, UCm, URT, I2, UCa $\}$

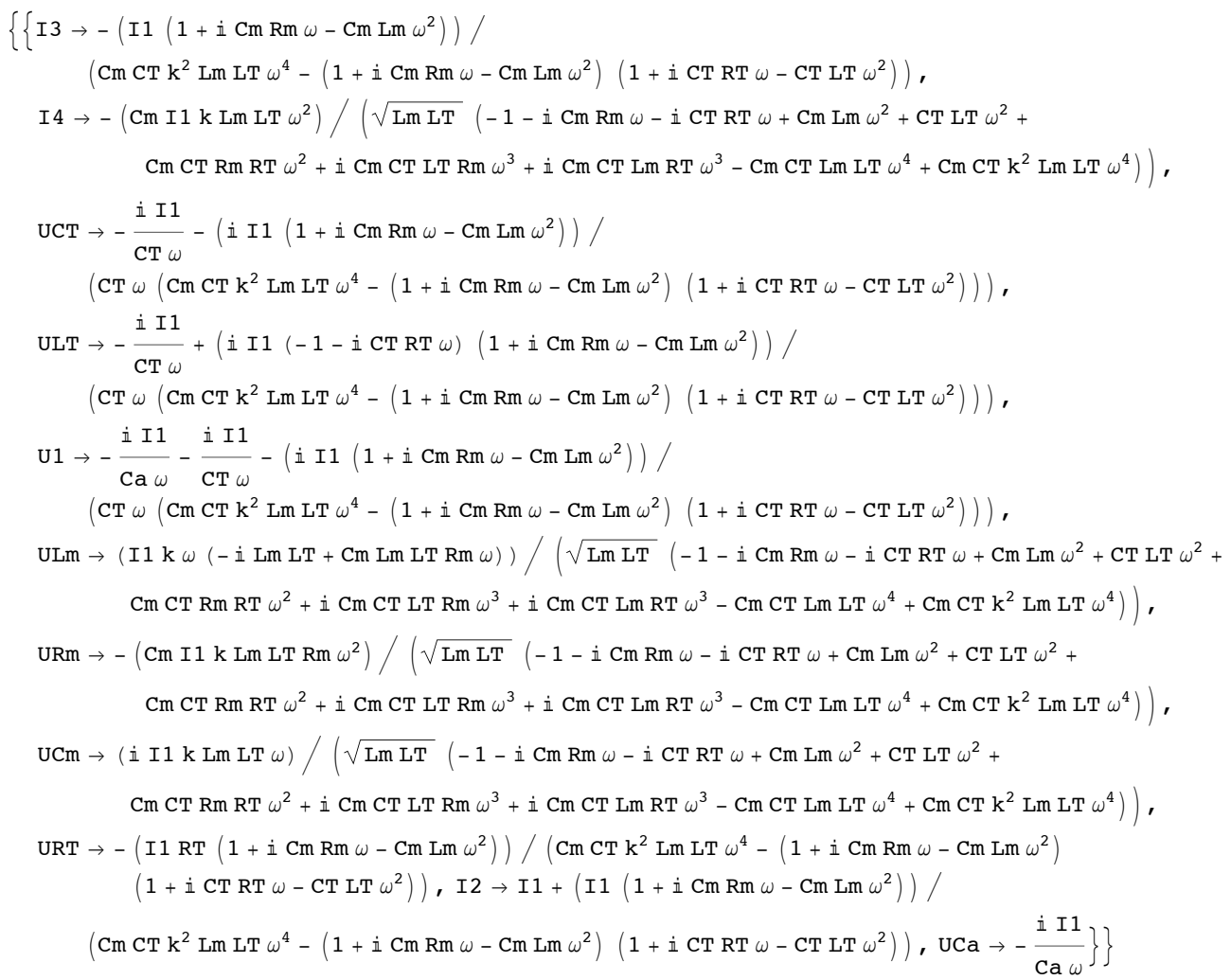

Figure A.2: Inductive coupling calculation part (b). 


\section{Power Dissipation}

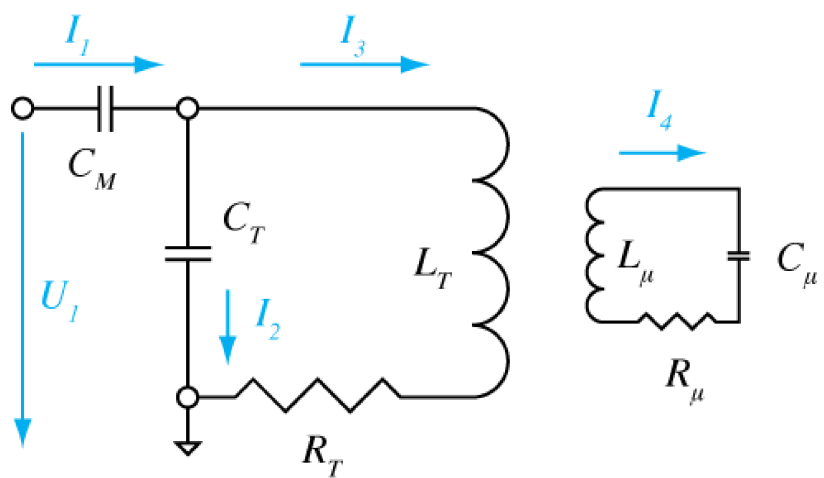

PowerProbe $=($ ComplexExpand $[$ I3 Conjugate [I3] RT //. solutions [1] ] ] // Simplify $)$

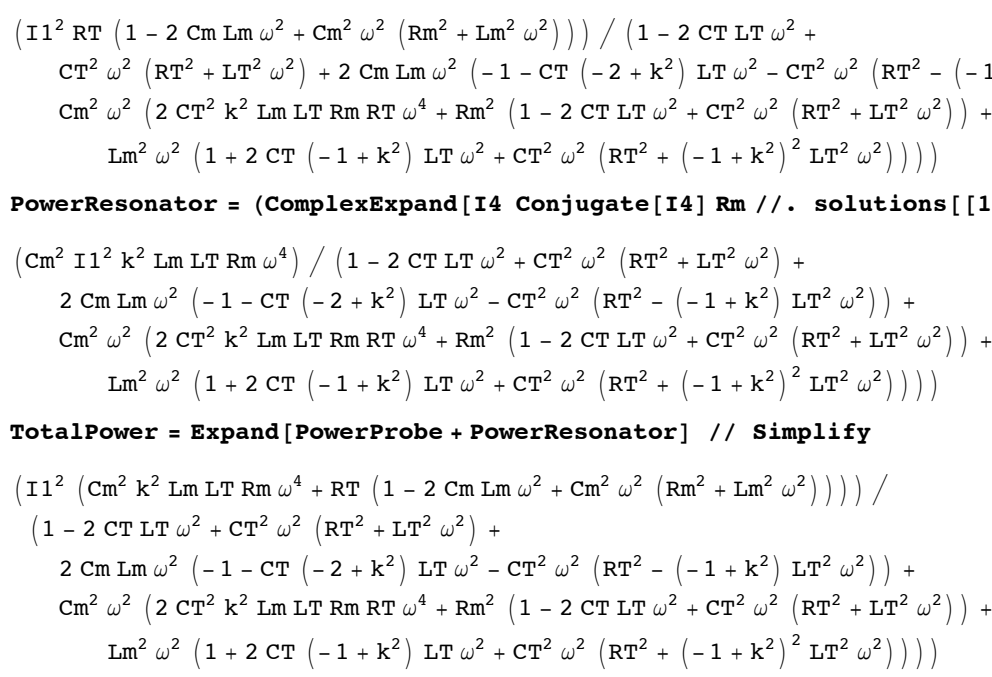

The total power dissipated should be equal to the power input to the system ComplexExpand[Re[U1] I1//. solutions[[1]]] // Simplify

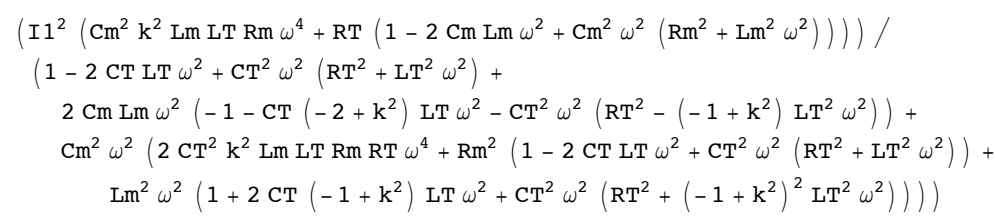

$\%=$ TotalPower

True

PowerEfficiency $=($ PowerResonator $/$ TotalPower $/ /$ Simplify $)$

$\left(\mathrm{Cm}^{2} \mathrm{k}^{2} \mathrm{LmLTRm} \omega^{4}\right) /\left(\mathrm{Cm}^{2} \mathrm{k}^{2} \operatorname{LmLTRm} \omega^{4}+\mathrm{RT}\left(1-2 \mathrm{Cm} \mathrm{Lm} \omega^{2}+\mathrm{Cm}^{2} \omega^{2}\left(\mathrm{Rm}^{2}+\mathrm{Lm}^{2} \omega^{2}\right)\right)\right)$

PowerEfficiency //. $\{\mathrm{Rm} \rightarrow \omega \mathrm{Lm} / \mathrm{Qm}, \mathrm{RT} \rightarrow \omega \mathrm{LT} / \mathbf{Q T}, \omega \rightarrow \Omega /$ Sqrt $[\mathrm{Lm} \mathrm{Cm}]\} / /$ Simplify

$\frac{\mathrm{k}^{2} \mathrm{Qm} \mathrm{QT} \Omega^{4}}{\Omega^{4}+\mathrm{k}^{2} \mathrm{Qm} \mathrm{QT} \Omega^{4}+\mathrm{Qm}^{2}\left(-1+\Omega^{2}\right)^{2}}$

$\% / . \Omega \rightarrow 1$

$\frac{\mathrm{k}^{2} \mathrm{Qm} \mathrm{QT}}{1+\mathrm{k}^{2} \mathrm{Qm} \mathrm{QT}}$

Figure A.3: Inductive coupling calculation part (c). 


\section{Reflection Ratio and Power Efficiency}

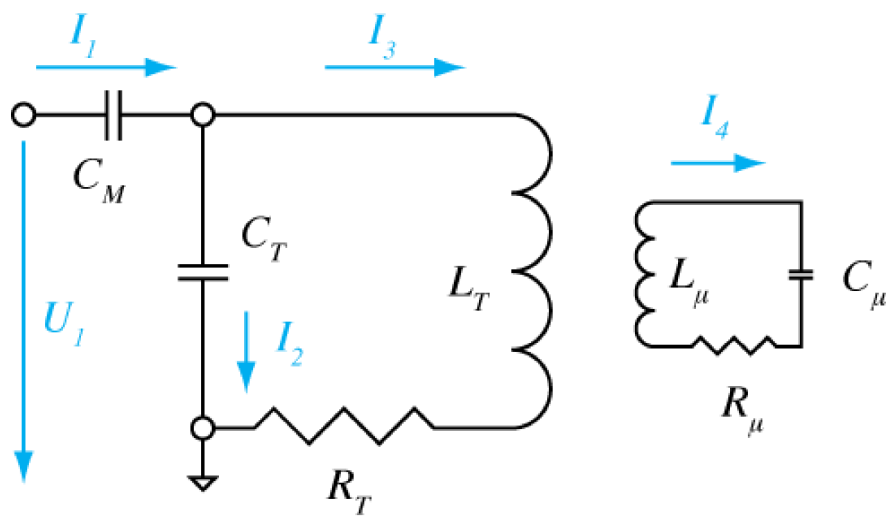

Manipulate [

GraphicsGrid [

$\{$ \{LogLinearPlot [

Evaluate $[20 / \log [10] \log [$ Abs [\#] ] \& /@ ( PowerEfficiency, $(U 1-50) /(U 1+50)\}) / /$. solutions //. $\{\mathrm{Ca} \rightarrow \mathrm{Cmatch}, \mathrm{Cm} \rightarrow \mathrm{Cmu}, \mathrm{Lm} \rightarrow 40 \mathrm{nH}, \mathrm{Rm} \rightarrow 2$,

$\mathrm{CT} \rightarrow \mathrm{Ct}, \mathrm{LT} \rightarrow 40 \mathrm{nH}, \mathrm{RT} \rightarrow 0.3, \omega \rightarrow 2 \pi \vee \mathrm{MHz}, \mathrm{I} 1 \rightarrow 1, \mathrm{k} \rightarrow \mathrm{coupl}\}]$

$\{v, 200,2000\}$, PlotRange $\rightarrow\{-20,5\}$, PlotPoints $\rightarrow 100$, PlotStyle $\rightarrow$ Thick,

Basestyle $\rightarrow$ \{FontFamily $\rightarrow$ "Helvetica", FontSize $\rightarrow 14$ \},

Frame $\rightarrow$ True, Framelabel $\rightarrow\{$ " $v / \mathrm{MHz} "$, "Gain/dB" $\}$, GridLines $\rightarrow$ True $]\}$

$\{\operatorname{LogLinearPlot}[E v a l u a t e[10 / \log [10] \log [\mathrm{Abs}[\#]] \& / @(\{I 3, I 4\}) / /$. solutions //.

$\{\mathrm{Ca} \rightarrow$ Cmatch $, \mathrm{Cm} \rightarrow \mathrm{Cmu}, \mathrm{Lm} \rightarrow 40 \mathrm{nH}, \mathrm{Rm} \rightarrow 2, \mathrm{CT} \rightarrow \mathrm{Ct}, \mathrm{LT} \rightarrow 40 \mathrm{nH}, \mathrm{RT} \rightarrow 0.3, \omega \rightarrow 2 \pi v \mathrm{MHz}$,

$I 1 \rightarrow 1, \mathbf{k} \rightarrow$ coupl $\}],\{v, 200,2000\}$, PlotRange $\rightarrow\{-10,30\}$, PlotPoints $\rightarrow 100$,

PlotStyle $\rightarrow$ Thick, Basestyle $\rightarrow$ \{FontFamily $\rightarrow$ "Helvetica", FontSize $\rightarrow 14\}$,

Frame $\rightarrow$ True, FrameLabel $\rightarrow\{$ "v/MHz", "Gain/dB" $\}]\}\}]$,

$\{C t, 0.001 \mathrm{pF}, 10 \mathrm{pF}\},\{\mathrm{Cmu}, 0.1 \mathrm{pF}, 10 \mathrm{pF}\}$,

\{Cmatch,

$0.01 \mathrm{pF}, 10 \mathrm{pF}\}$,

$\{$ coupl, $0.0001,0.2\}] / /$. Units

Figure A.4: Inductive coupling calculation part (d). 


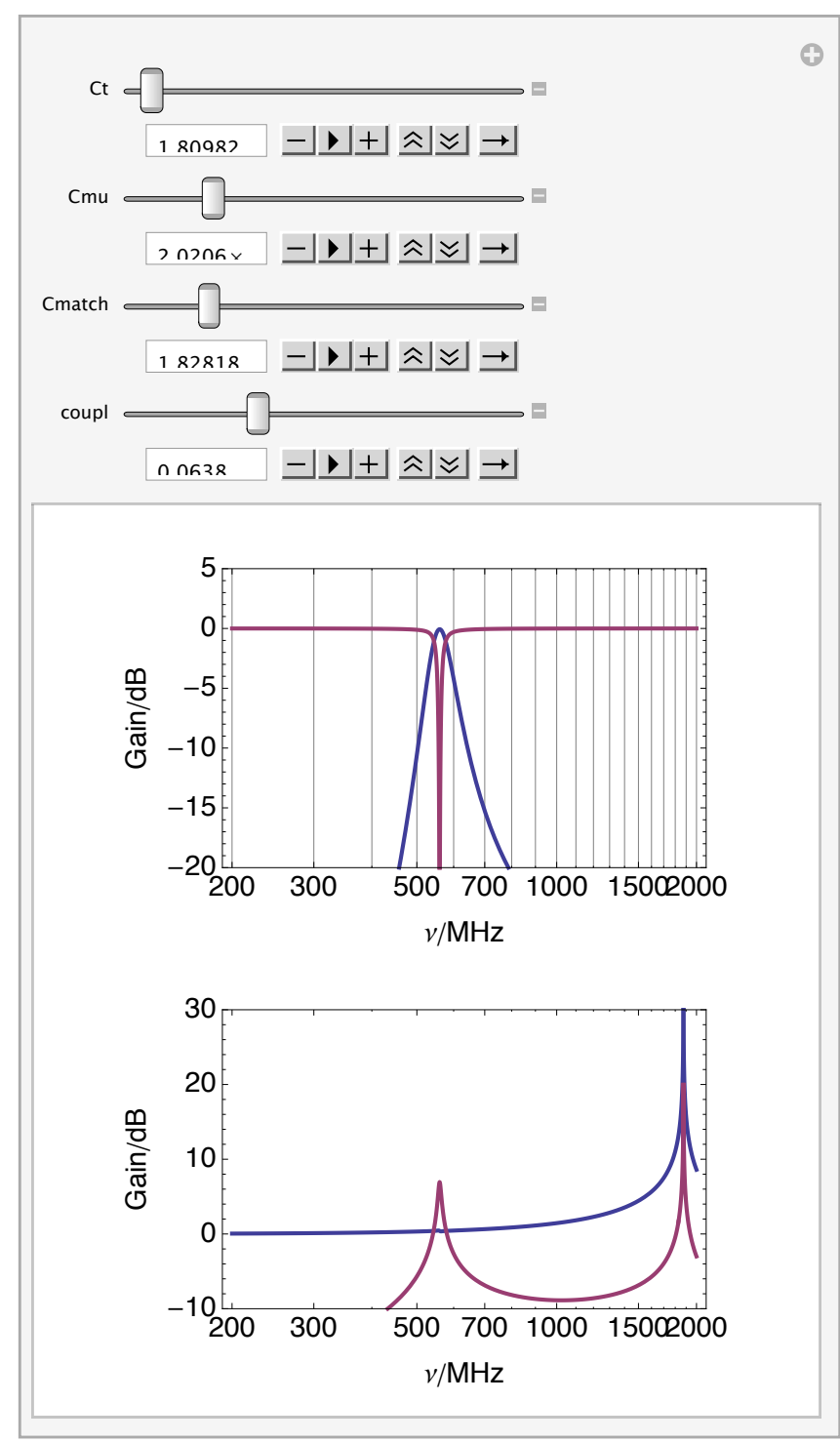

Figure A.5: Inductive coupling calculation part (e). 


\section{Current Resonance in Primary Coil and Resonator}

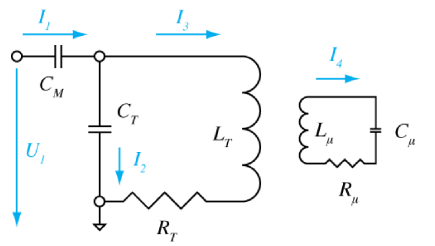

Units $=\left\{\mathrm{MHz} \rightarrow 10^{\wedge} 6, \mathrm{pF} \rightarrow 10^{\wedge}(-12), \mathrm{nH} \rightarrow 10^{\wedge}(-9)\right\}$;

Manipulate [LogLinearPlot [Evaluate [

$10 / \log [10] \log [\mathrm{Abs}[\#]] \& / @(\{I 3, \mathrm{I} 4\}) / /$. solutions //. $\{\mathrm{Ca} \rightarrow \mathrm{Cmatch}, \mathrm{Cm} \rightarrow \mathrm{Cmu}$

$\mathrm{Lm} \rightarrow 40 \mathrm{nH}, \mathrm{Rm} \rightarrow 4, \mathrm{CT} \rightarrow \mathrm{Ct}, \mathrm{LT} \rightarrow 20 \mathrm{nH}, \mathrm{RT} \rightarrow 0.3, \omega \rightarrow 2 \pi v \mathrm{MHz}$, I $1 \rightarrow 1, \mathrm{k} \rightarrow$ coupl $\}]$, $\{v, 200,2000\}$, PlotRange $\rightarrow\{-10,30\}$, PlotPoints $\rightarrow 100$, PlotStyle $\rightarrow$ Thick,

BaseStyle $\rightarrow$ FontFamily $\rightarrow$ "Helvetica", FontSize $\rightarrow 14\}$, Frame $\rightarrow$ True,

FrameLabel $\rightarrow\{" v / M H z ", "$ Gain $/ \mathrm{dB} "\}],\{\mathrm{Ct}, 0.1 \mathrm{pF}, 10 \mathrm{pF}\}$,

$\{\mathrm{Cmu}, 0.1 \mathrm{pF}, 10 \mathrm{pF}\},(*\{$ Cmatch, $0.01 \mathrm{pF}, 20 \mathrm{pF}\}, *)\{$ coupl $, 0.0001,0.2\}] / /$. Units

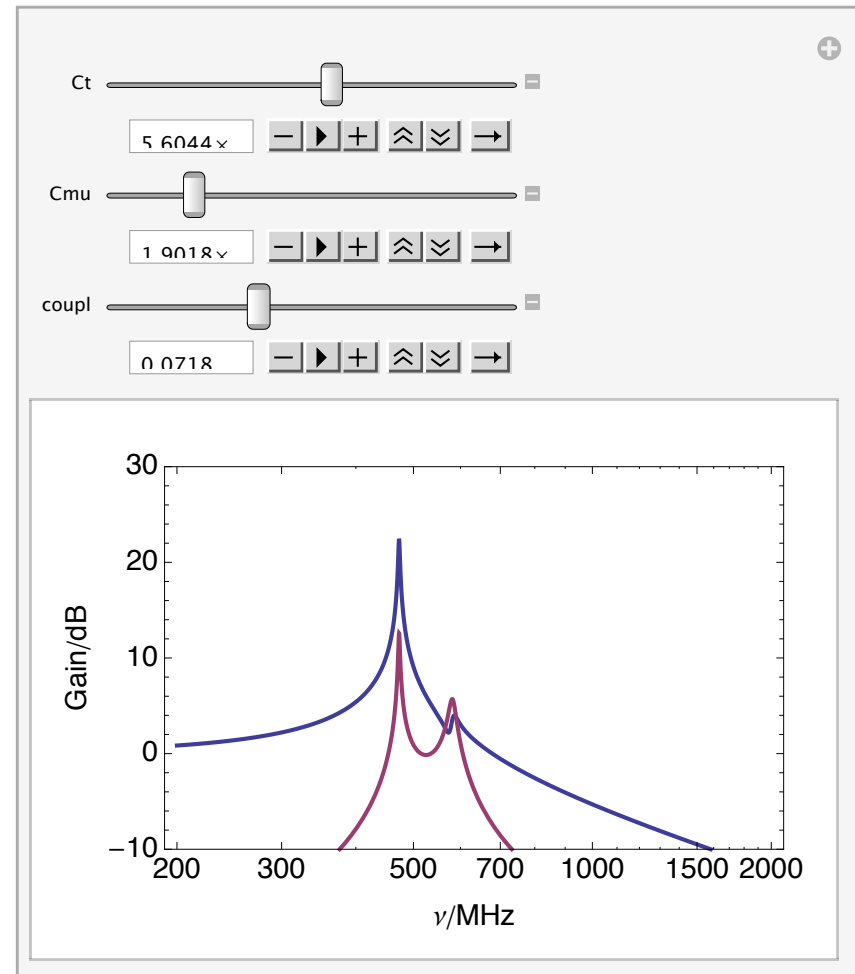

Figure A.6: Inductive coupling calculation part (f). 
Appendix B

Mathematica Coil Calculations 


\section{Flat Spiral Coils}

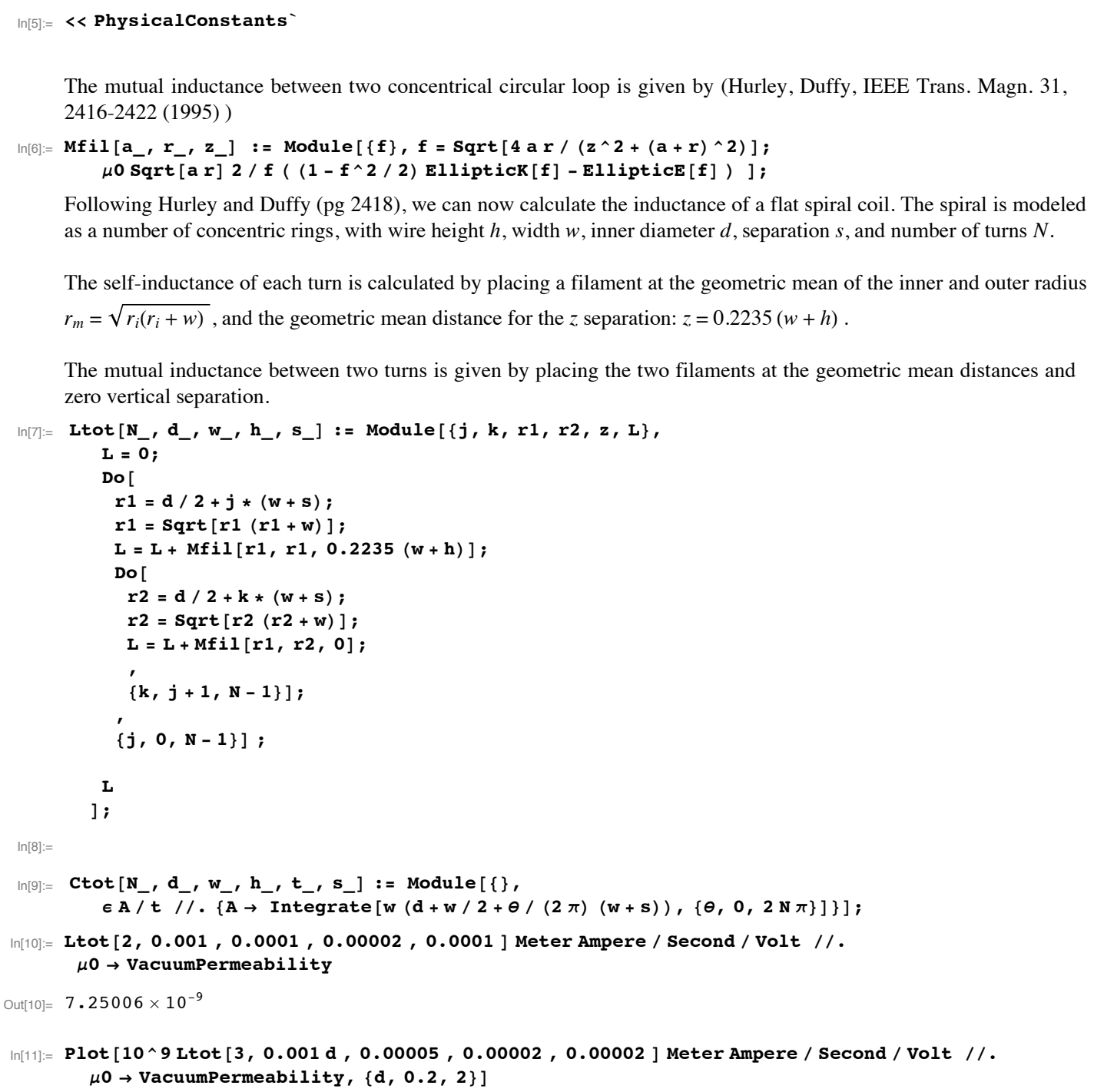

The mutual inductance between two concentrical circular loop is given by (Hurley, Duffy, IEEE Trans. Magn. 31, 2416-2422 (1995))

Following Hurley and Duffy (pg 2418), we can now calculate the inductance of a flat spiral coil. The spiral is modeled as a number of concentric rings, with wire height $h$, width $w$, inner diameter $d$, separation $s$, and number of turns $N$.

The self-inductance of each turn is calculated by placing a filament at the geometric mean of the inner and outer radius $r_{m}=\sqrt{r_{i}\left(r_{i}+w\right)}$, and the geometric mean distance for the $z$ separation: $z=0.2235(w+h)$.

The mutual inductance between two turns is given by placing the two filaments at the geometric mean distances and zero vertical separation.

\section{Coil Resonator Simulation}

We assume the coil to be fabricated on top of a dielectric layer of thickness $t$, which separates it from another conducting layer.

Figure B.1: Microcoil inductance calculations part (a). 


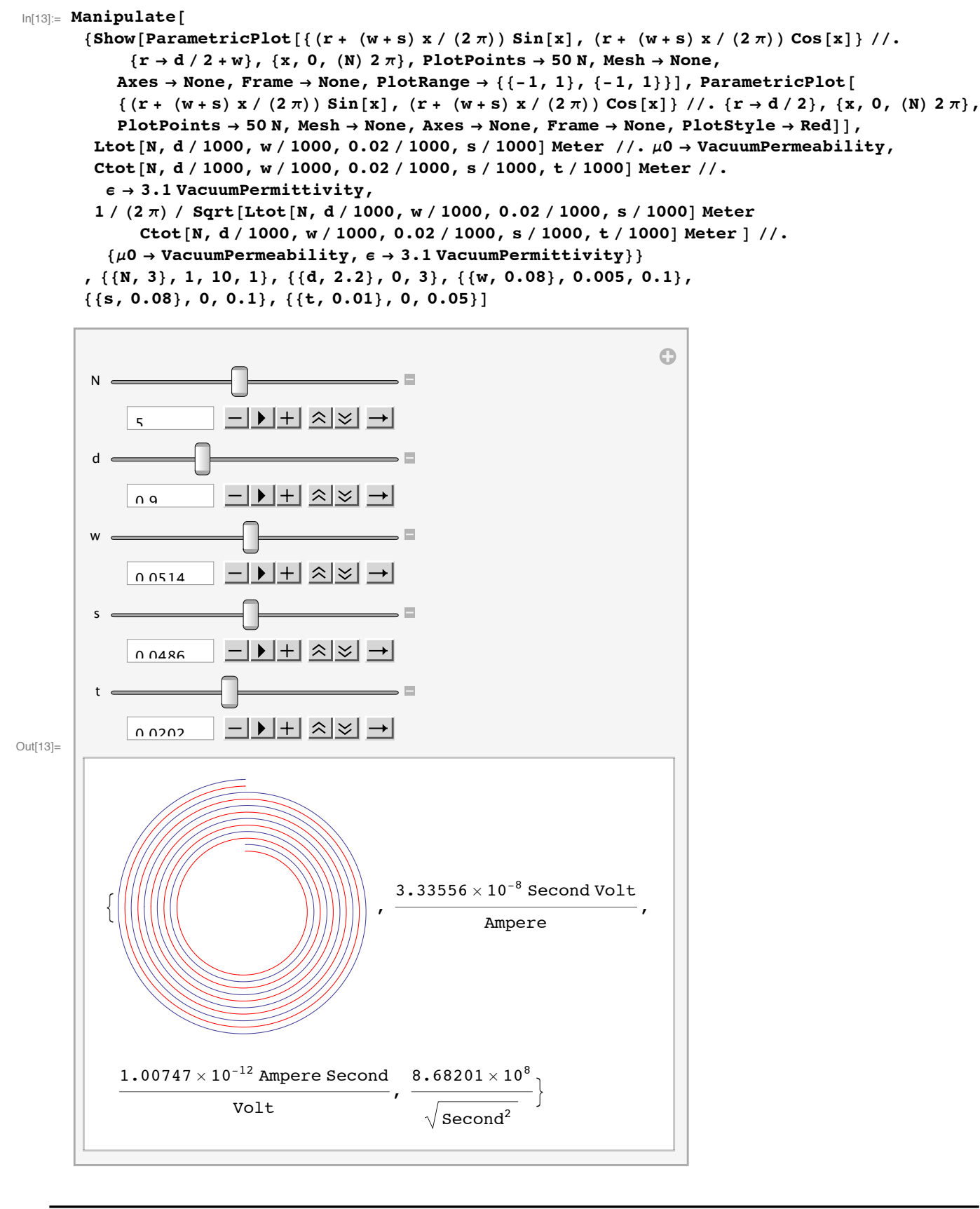

Figure B.2: Microcoil inductance calculations part (b). 


\section{Wheeler Formula (Inductance)}

Herbert Ryan

$6 / 21 / 2011$

Clear [ "Global * *"]

- Geometrical Parameters

Relative magnetic permeability of free space, $S U-8=1.08$

$\mathrm{mu}=1.08$;

Magnetic permeability of free space $\left[H / m=N / A^{2}=T m / A\right]$

muo $=1.257 \times 10^{-6} ;$

Number of turns

$\mathrm{Nu}=4$;

Coil Width [m]

$\mathrm{w}=100 \times 10^{-6} ;$

spacing [m]

$\mathbf{s}=100 \times 10^{-6}$;

outer radius $[\mathrm{m}]$

ro $=5 \times 10^{-3}$;

inner radius $[\mathrm{m}]$

$r i=N[r o-N u w-(N u-1) s]$

0.0043

Inductance $[\mathrm{nH}]$

$\mathrm{L}=\mathrm{mu}$ muo $\mathrm{Nu}^{2}\left((\mathrm{ro}+\mathrm{ri}+\mathrm{w})^{2} /(2.14 \mathrm{ro}-\mathrm{ri}+0.57 \mathrm{w})\right) 10^{9}$

297.238

- Intrinsic Capactiance

relative permittivity, $\mathrm{SU}-8=3.2$, glass $=4.6[\mathrm{~F} / \mathrm{m}]$

$\epsilon r=3.2$;

Permittivity of free space (vacuum)

$\epsilon O=8.85 \times 10^{-12} ;$

Capacitance $[\mathrm{pF}]$

$\mathrm{Ca}=\frac{\pi^{2} \in 0 \in \mathrm{r}(\mathrm{ri}+\mathrm{ro}) 10^{12}}{\operatorname{ArcCosh}[(\mathrm{s}+\mathrm{w}) / 2 / \mathrm{w}(\mathrm{Nu}-1)]}+70 \mathrm{ro}+0.06$

1.88464

- Comb Capacitor Capactiance

Capcitance

Figure B.3: Wheeler formula coil calculations part (c). 


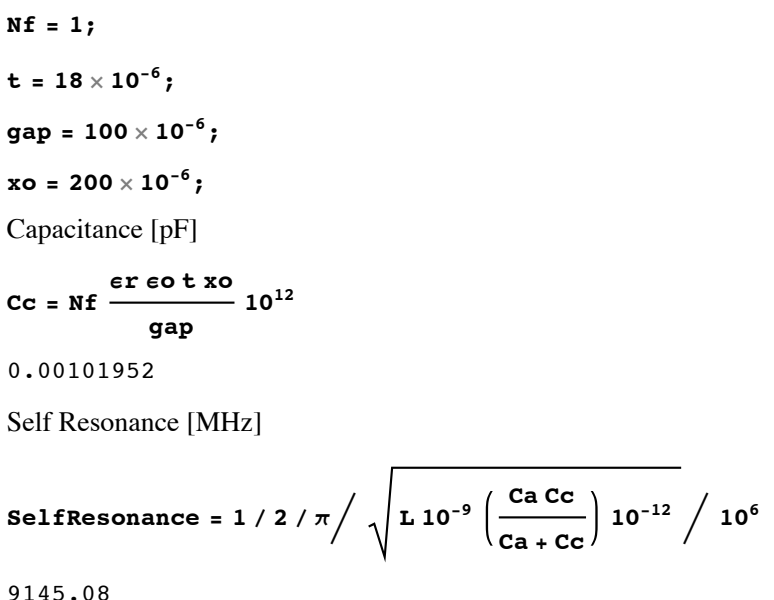

- Helmboltz Coil (two microcoils) ------ Not Used for microcoil

- Magnetic field induction along the axis

$\mathrm{x}$ distance

$x=$ Table $[i,\{i, 0$, ro, ro $/ 100\}]$;

num $=\frac{\text { muo }}{2} \mathrm{Nu} \mathrm{ro}{ }^{2}$

$6.285 \times 10^{-11}$

Total field on the axis of a pair of helmboltz coils

$\operatorname{den}=\mathbf{N}\left[\frac{1}{\left(r \mathrm{r}^{2}+(\mathrm{x}-\mathrm{ro})^{2}\right)^{\frac{3}{2}}}+\frac{1}{\left(\mathrm{ro}^{2}+\mathrm{x}^{2}\right)^{\frac{3}{2}}}\right]$

$\left\{1.08284 \times 10^{7}, 1.087 \times 10^{7}, 1.09098 \times 10^{7}, 1.09478 \times 10^{7}, 1.09841 \times 10^{7}\right.$,

$1.10187 \times 10^{7}, 1.10516 \times 10^{7}, 1.10828 \times 10^{7}, 1.11124 \times 10^{7}, 1.11404 \times 10^{7}, 1.11668 \times 10^{7}$, $1.11916 \times 10^{7}, 1.12149 \times 10^{7}, 1.12367 \times 10^{7}, 1.12571 \times 10^{7}, 1.12762 \times 10^{7}, 1.12938 \times 10^{7}$, $1.13102 \times 10^{7}, 1.13253 \times 10^{7}, 1.13393 \times 10^{7}, 1.1352 \times 10^{7}, 1.13637 \times 10^{7}, 1.13744 \times 10^{7}$ $1.1384 \times 10^{7}, 1.13928 \times 10^{7}, 1.14006 \times 10^{7}, 1.14076 \times 10^{7}, 1.14139 \times 10^{7}, 1.14194 \times 10^{7}$, $1.14242 \times 10^{7}, 1.14285 \times 10^{7}, 1.14322 \times 10^{7}, 1.14353 \times 10^{7}, 1.1438 \times 10^{7}, 1.14403 \times 10^{7}$, $1.14422 \times 10^{7}, 1.14437 \times 10^{7}, 1.1445 \times 10^{7}, 1.1446 \times 10^{7}, 1.14468 \times 10^{7}, 1.14474 \times 10^{7}$, $1.14478 \times 10^{7}, 1.14481 \times 10^{7}, 1.14484 \times 10^{7}, 1.14485 \times 10^{7}, 1.14486 \times 10^{7}, 1.14486 \times 10^{7}$, $1.14487 \times 10^{7}, 1.14487 \times 10^{7}, 1.14487 \times 10^{7}, 1.14487 \times 10^{7}, 1.14487 \times 10^{7}, 1.14487 \times 10^{7}$, $1.14487 \times 10^{7}, 1.14486 \times 10^{7}, 1.14486 \times 10^{7}, 1.14485 \times 10^{7}, 1.14484 \times 10^{7}, 1.14481 \times 10^{7}$, $1.14478 \times 10^{7}, 1.14474 \times 10^{7}, 1.14468 \times 10^{7}, 1.1446 \times 10^{7}, 1.1445 \times 10^{7}, 1.14437 \times 10^{7}$, $1.14422 \times 10^{7}, 1.14403 \times 10^{7}, 1.1438 \times 10^{7}, 1.14353 \times 10^{7}, 1.14322 \times 10^{7}, 1.14285 \times 10^{7}$, $1.14242 \times 10^{7}, 1.14194 \times 10^{7}, 1.14139 \times 10^{7}, 1.14076 \times 10^{7}, 1.14006 \times 10^{7}, 1.13928 \times 10^{7}$, $1.1384 \times 10^{7}, 1.13744 \times 10^{7}, 1.13637 \times 10^{7}, 1.1352 \times 10^{7}, 1.13393 \times 10^{7}, 1.13253 \times 10^{7}$, $1.13102 \times 10^{7}, 1.12938 \times 10^{7}, 1.12762 \times 10^{7}, 1.12571 \times 10^{7}, 1.12367 \times 10^{7}, 1.12149 \times 10^{7}$, $1.11916 \times 10^{7}, 1.11668 \times 10^{7}, 1.11404 \times 10^{7}, 1.11124 \times 10^{7}, 1.10828 \times 10^{7}, 1.10516 \times 10^{7}$, $\left.1.10187 \times 10^{7}, 1.09841 \times 10^{7}, 1.09478 \times 10^{7}, 1.09098 \times 10^{7}, 1.087 \times 10^{7}, 1.08284 \times 10^{7}\right\}$

Magnetic field current ratio (T/A), Magnetic field (Bx) [Tesla]

FiguRE B.4: Wheeler formula coil calculations part (b). 


\begin{abstract}
$\mathrm{Bxi}=$ num den
$\{0.000680567,0.000683178,0.000685679,0.00068807,0.000690352$,

$0.000692526,0.000694594,0.000696556,0.000698416,0.000700173,0.000701831$, $0.000703391,0.000704857,0.000706229,0.000707511,0.000708707,0.000709817$, $0.000710846,0.000711797,0.000712673,0.000713476,0.000714211,0.00071488$, $0.000715487,0.000716035,0.000716528,0.000716969,0.000717361,0.000717708$, $0.000718013,0.00071828,0.000718511,0.000718709,0.000718878,0.000719021$, $0.000719139,0.000719237,0.000719316,0.00071938,0.000719429,0.000719467$, $0.000719495,0.000719515,0.000719529,0.000719538,0.000719544,0.000719547$, $0.000719548,0.000719549,0.000719549,0.000719549,0.000719549,0.000719549$, $0.000719548,0.000719547,0.000719544,0.000719538,0.000719529,0.000719515$, $0.000719495,0.000719467,0.000719429,0.00071938,0.000719316,0.000719237$, $0.000719139,0.000719021,0.000718878,0.000718709,0.000718511,0.00071828$, $0.000718013,0.000717708,0.000717361,0.000716969,0.000716528,0.000716035$, $0.000715487,0.00071488,0.000714211,0.000713476,0.000712673,0.000711797$, $0.000710846,0.000709817,0.000708707,0.000707511,0.000706229,0.000704857$, $0.000703391,0.000701831,0.000700173,0.000698416,0.000696556,0.000694594$, $0.000692526,0.000690352,0.00068807,0.000685679,0.000683178,0.000680567\}$
\end{abstract}

$d=N[T a b l e[i,\{i,-r o / 2, r o / 2, r o / 100\}]]$

$\{-0.0025,-0.00245,-0.0024,-0.00235,-0.0023,-0.00225,-0.0022,-0.00215,-0.0021$, $-0.00205,-0.002,-0.00195,-0.0019,-0.00185,-0.0018,-0.00175,-0.0017,-0.00165$, $-0.0016,-0.00155,-0.0015,-0.00145,-0.0014,-0.00135,-0.0013,-0.00125$,

$-0.0012,-0.00115,-0.0011,-0.00105,-0.001,-0.00095,-0.0009,-0.00085$,

$-0.0008,-0.00075,-0.0007,-0.00065,-0.0006,-0.00055,-0.0005,-0.00045$,

$-0.0004,-0.00035,-0.0003,-0.00025,-0.0002,-0.00015,-0.0001,-0.00005,0$. $0.00005,0.0001,0.00015,0.0002,0.00025,0.0003,0.00035,0.0004,0.00045,0.0005$, $0.00055,0.0006,0.00065,0.0007,0.00075,0.0008,0.00085,0.0009,0.00095,0.001$, $0.00105,0.0011,0.00115,0.0012,0.00125,0.0013,0.00135,0.0014,0.00145,0.0015$, $0.00155,0.0016,0.00165,0.0017,0.00175,0.0018,0.00185,0.0019,0.00195,0.002$ ， $0.00205,0.0021,0.00215,0.0022,0.00225,0.0023,0.00235,0.0024,0.00245,0.0025\}$ $\mathrm{x}[\mathrm{mm}], \mathrm{Bx}[\mathrm{mT} / \mathrm{A}]$

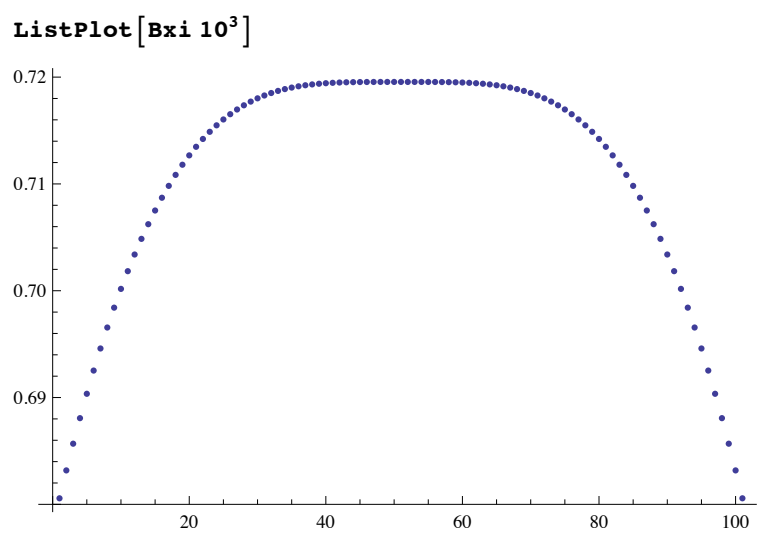

FigURE B.5: Wheeler formula coil calculations part . 
Appendix C

Microfabrication Masks 
ए

$\eta$
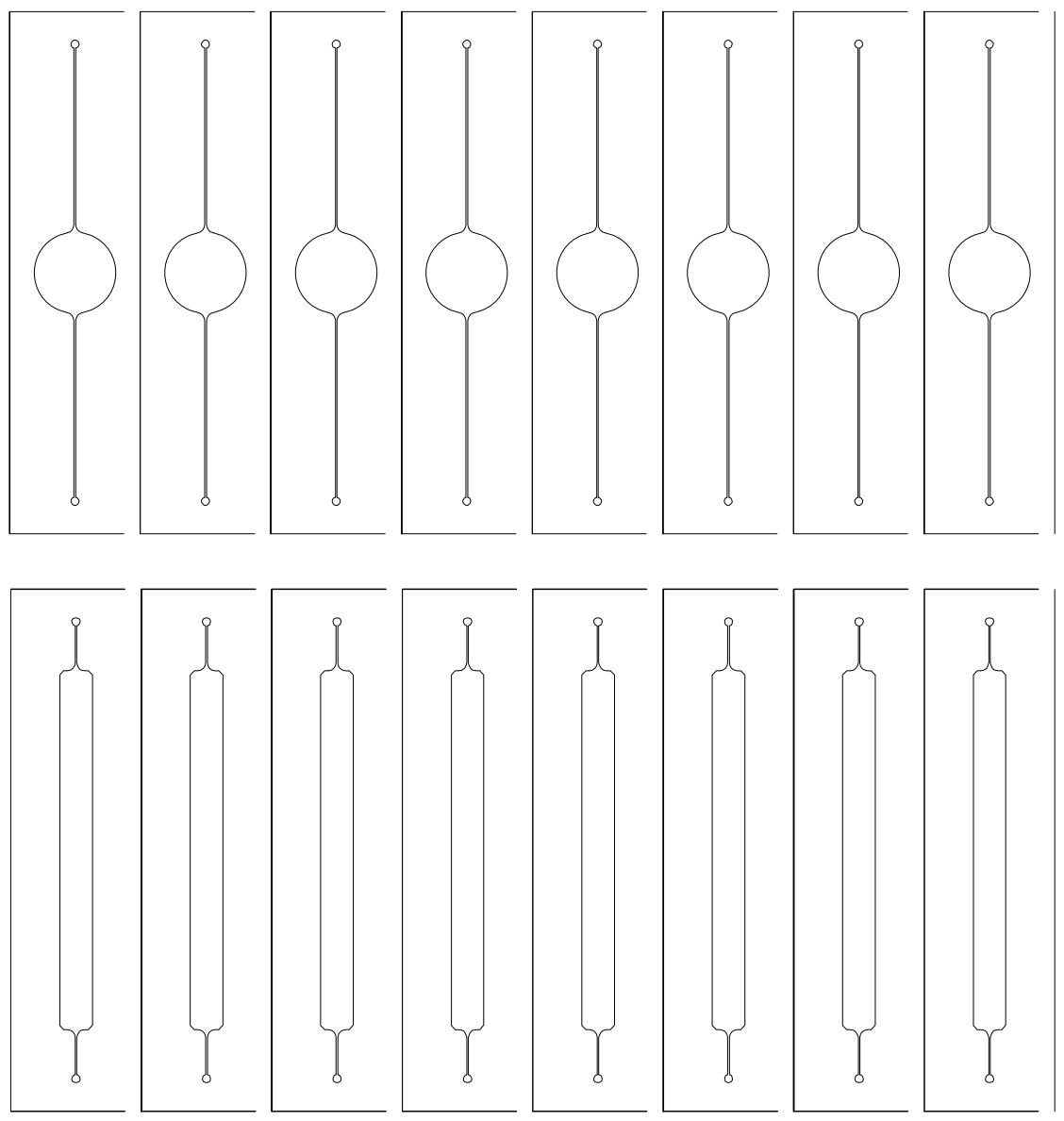

$\longleftarrow$

Figure C.1: Microfluidic chip mask. 


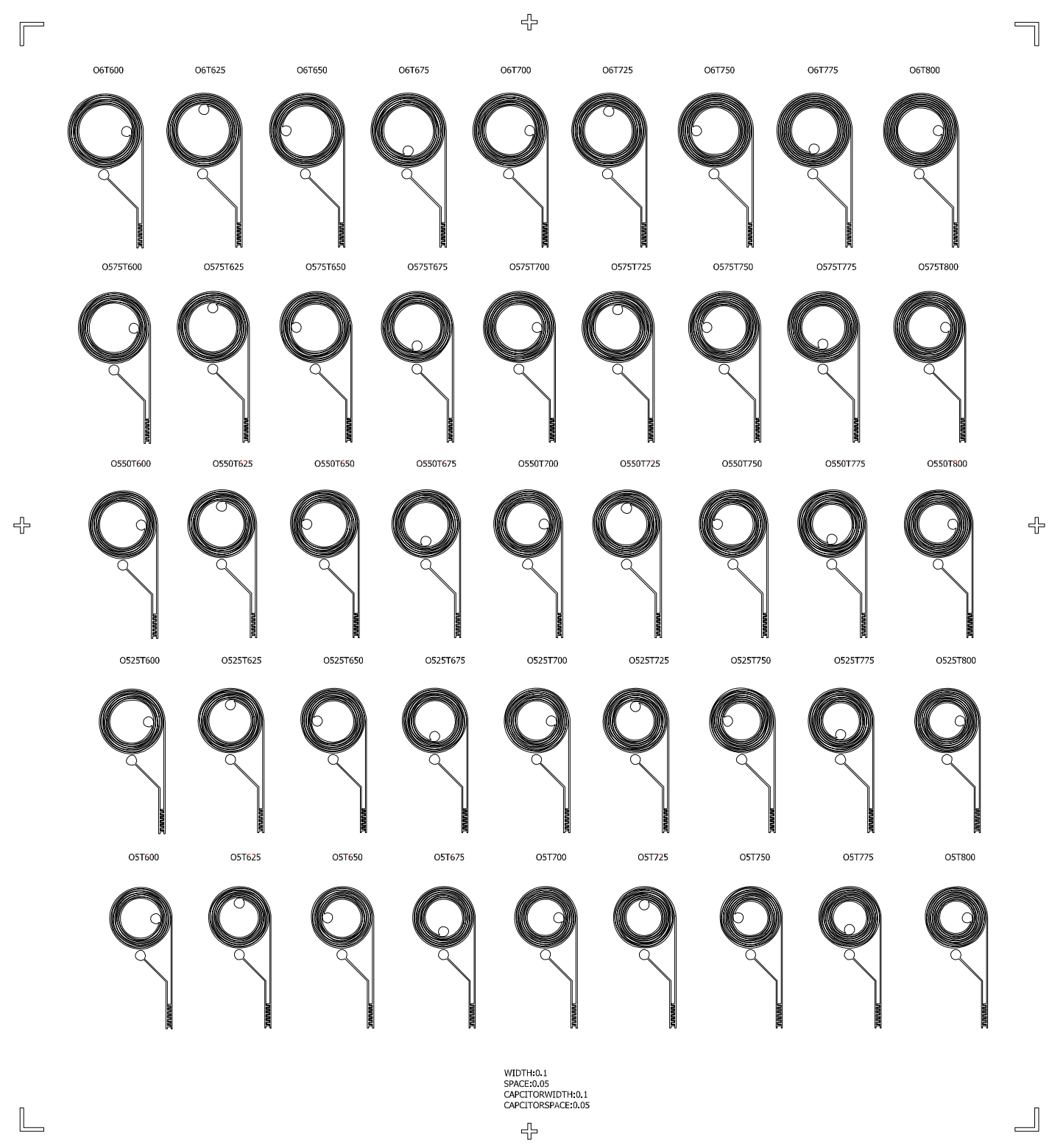

FiguRE C.2: Microcoil resonator mask 1. 


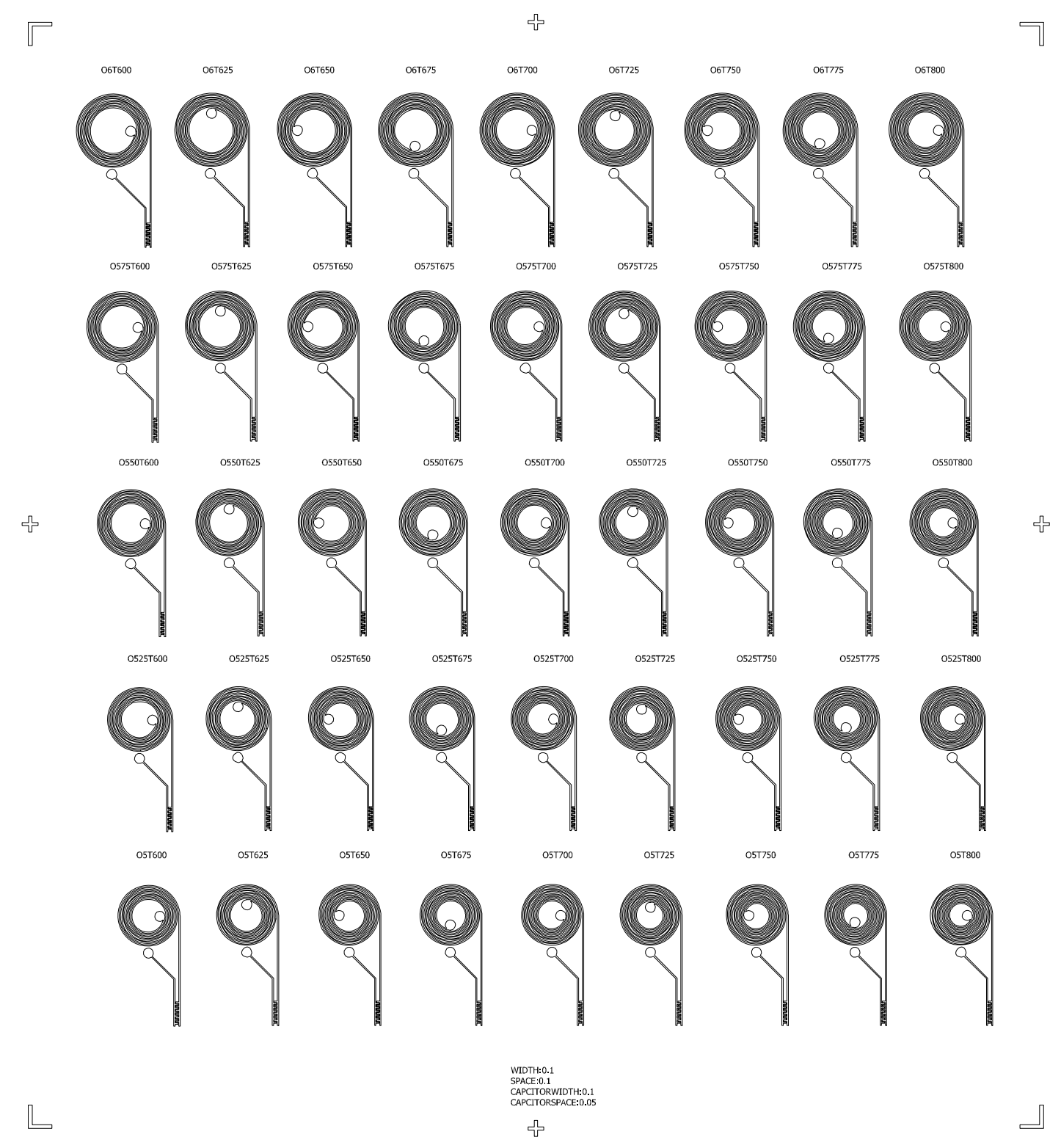

Figure C.3: Microcoil resonator mask 1. 


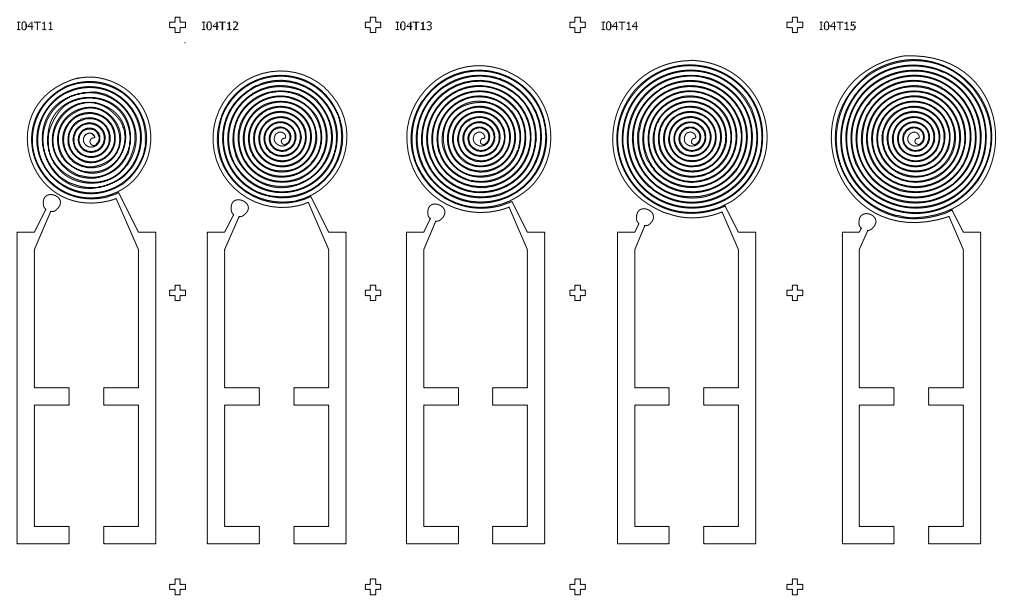

$\longleftarrow$

Figure C.4: Adjustable microcoil resonator mask. 
Appendix D

Mathematica Network Analyzer

Data Processing 


\section{HP8753 Data}

\section{HP8753d-2206c Processing}

- HP8753d-2206c Probe $S_{11}$ and Microcoil $S_{22}$ Ice Cream Cone SetDirectory [" /Desktop/Research/Microfluidic_NMR/data"]

/home/hbr3e/Desktop/Research/Microfluidic_NMR/data

data1 = Import $[$ "hp8753d-2206c/7-15-2011/4coil18s.d1"];

data2 = Import ["hp8753d-2206c/7-15-2011/4coil19s.d1"];

data3 = Import ["hp8753d-2206c/circuit_245c.d1"];

HP8753Plot [data_, options__] := Module [ start, stop, $\mathrm{n}$, values ,

start $=$ ToExpression $[$ StringSplit $[\operatorname{data}[[7,1]]][[2]]] / 10^{\wedge} \mathbf{6}_{\text {; }}$

stop $=\operatorname{ToExpression}[\operatorname{StringSplit}[\operatorname{data}[[7,1]]][[3]]] / 10^{\wedge} 6_{\text {; }}$

$\mathrm{n}=$ ToExpression $[$ StringSplit $[\operatorname{data}[[7,1]]]][[4]]$;

values = Table $[$ start $+\mathbf{k}$ (stop - start) $/ \mathbf{n}$,

$\left.\left.\operatorname{data}[[k+9]] / .\left\{a_{-}, b_{-}\right\} \rightarrow 20 / \log [10] \log [\operatorname{Abs}[a+I b]]\right\},\{k, 1, \mathbf{n}\}\right] ;$ ListPlot [values, options] ]

Show [HP8753Plot [data1, Joined $\rightarrow$ True, PlotRange $\rightarrow\{0,-40\}$, PlotStyle $\rightarrow\{$ Darker [Green] $\}]$, HP8753Plot [data2, Joined $\rightarrow$ True, PlotStyle $\rightarrow\{\{$ Thick, Dashed $\}\}]$, HP8753Plot [data3, Joined $\rightarrow$ True, PlotStyle $\rightarrow\{$ Red $\}$ ],

Frame $\rightarrow$ True, AspectRatio $\rightarrow 1$, BaseStyle $\rightarrow$ \{FontFamily $\rightarrow$ "Helvetica", FontSize $\rightarrow 14$ \} Axes $\rightarrow$ None, Framelabel $\left.\rightarrow\left\{v[\mathrm{MHz}], \mathbf{S}_{11}[\mathrm{~dB}]\right\}\right]$

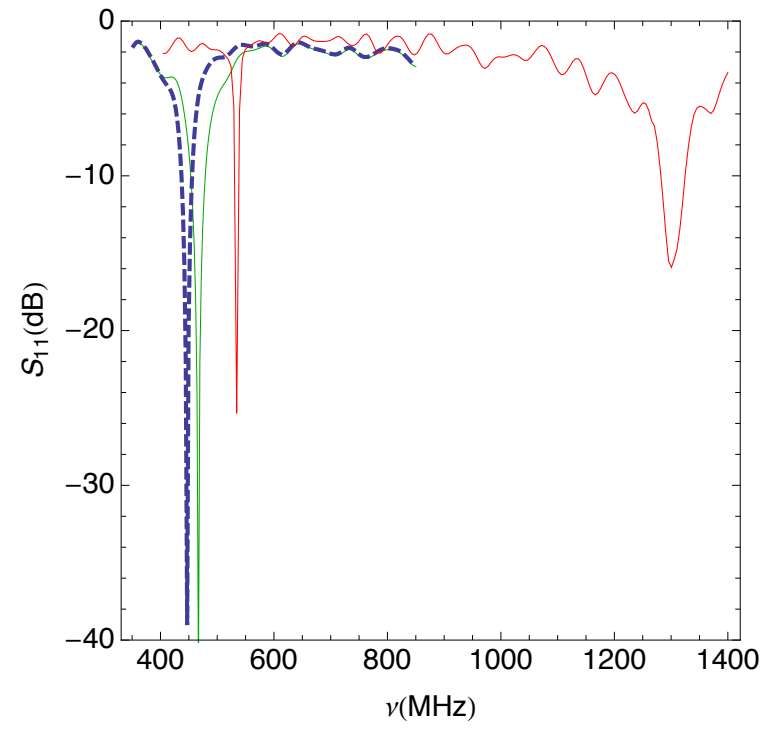

FiguRE D.1: Microcoil data processing part (a). 


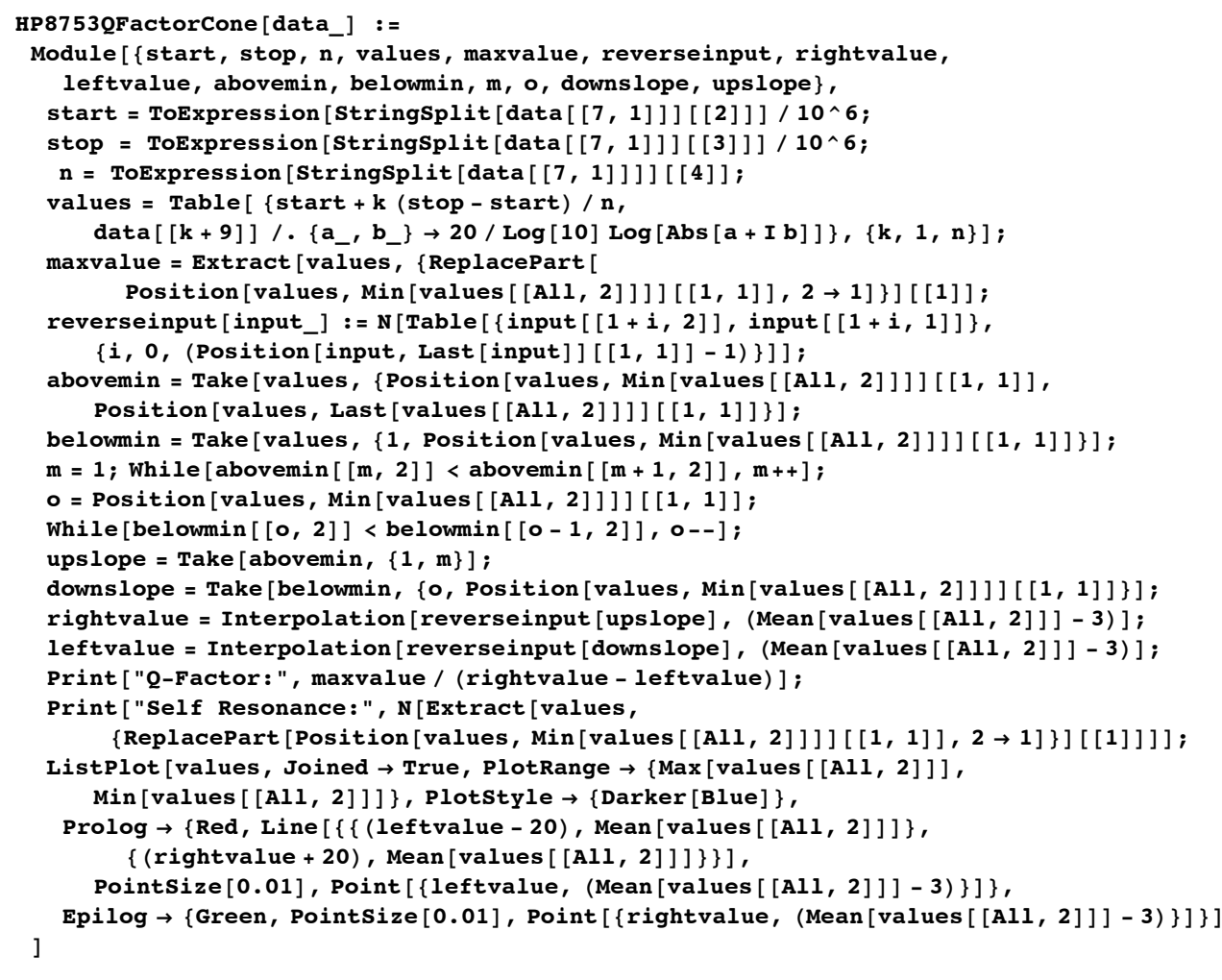

N [ HP8753QFactorCone [data2] ]

Q-Factor: 12.8603

Self Resonance: 447.015

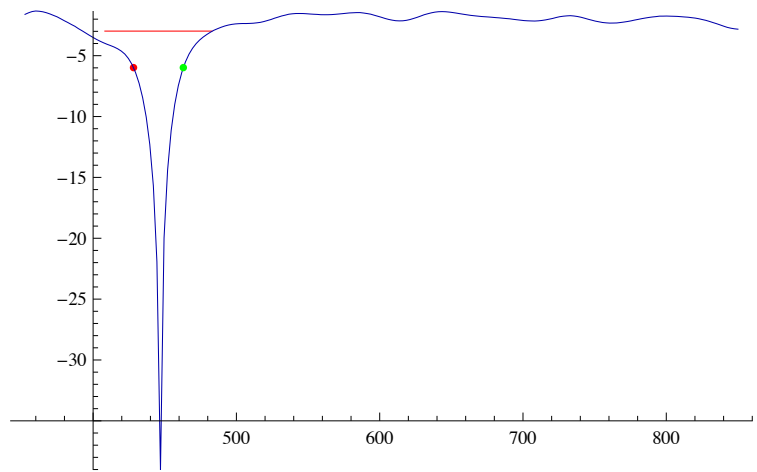

- Alternate Calculation

HP8753QFactorCone [data_] : =

Module [ start, stop, n, values, maxvalue, rightvalue, leftvalue\},

start $=$ ToExpression $[$ StringSplit $[\operatorname{data}[[7,1]]][[2]]] / 10^{\wedge} 6$;

stop $=$ ToExpression $[\operatorname{StringSplit}[\operatorname{data}[[7,1]]][[3]]] / 10^{\wedge} 6$;

$\mathbf{n}=$ ToExpression $[$ StringSplit $[\operatorname{data}[[7,1]]]][[4]]$;

Figure D.2: Microcoil data processing part (b). 
values = Table $[$ \{start $+\mathbf{k}$ (stop - start $) / \mathbf{n}$,

$\left.\left.\operatorname{data}[[\mathbf{k}+9]] / .\left\{a_{-}, b_{-}\right\} \rightarrow 20 / \log [10] \log [\operatorname{Abs}[a+I b]]\right\},\{k, 1, n\}\right] ;$

maxvalue $=$ Extract $[$ values, $\{$ ReplacePart [

Position [values, Min[values [ [Al1, 2] ] ] [ [1, 1]], 2 $\rightarrow 1]\}][[1]]$; rightvalue $=$

(*find if an acutal data point is less than average-

$3 \mathrm{~dB}$. absolute values on all values $*$ )

(If [Abs [Mean [values [ [Al1, 2] ] ] - 3] > Abs [Nearest [Take [values [ [All, 2]], \{Position [values, Min[values[[All, 2] ] ] $[[1,1]]$, Position[values, Last [values [ [Al1, 2] ] ] [ [1, 1]]\}], (Mean [values [ [Al1, 2] ] - 3)] ] [ [1] ], (*If true than interpolate to get a resonance data point at average-dB*) Interpolation [values [ $[A 11,1]]$,

(Position [values, Nearest [Take $[$ values $[$ All, 2] ], \{Position[values, Min [ values $[[A 11,2]]]][[1,1]]$, Position[values, Last [values [[All, 2] ] ] [ [ $1,1]]\}],($ Mean [values [ [Al1, 2] ] -3$)][[1]]][[1,1]]-$

( (Abs [Mean [values [ [Al1, 2] ] ] - 3] - Abs [Extract[values, \{Position[values, Nearest [ Take [values [ [Al1, 2] ], \{Position [values, Min[values [[All, 2]]]] [

$1,1]]$, Position [values, Last [values $[[A 11,2]]]][[1,1]]\}]$, (Mean[values [ [Al1, 2] ] ] 3)] [[1]]][[1]]\}][[1]]])/

(Abs [Extract [values, \{Position[values, Nearest [Take [values $[[A 11,2]]$, \{Position [values, $\operatorname{Min}[\operatorname{values}[[\mathrm{All}, 2]]]][[1,1]]$, Position [values, Last [values $[[A 11,2]]]][[1,1]]\}]$,

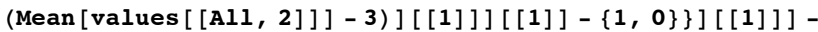

Abs [Extract [values, \{Position [values, Nearest [Take [values $[[A 11,2]]$, $\{$ Position [values, $\operatorname{Min}[\operatorname{values}[[\mathrm{All}, 2]]]][[1,1]]$, Position [values, Last [values $[[A 11,2]]]][[1,1]]\}]$ (Mean [values [ [Al1, 2]]]-3)][[1]]][[1]]\}][[1]]]))) ], Interpolation [values [ [All, 1] , (Position[values, Nearest[Take[

values $[$ [Al1, 2] $],\{$ Position[values, $\operatorname{Min}[\operatorname{values}[[A 11,2]]]][[1,1]]$, Position [values, Last [values $[[A 11,2]]]][[1,1]]\}]$,

(Mean [values [ [Al1, 2] ] ] - 3) ] [ [1] ] ] [ $[1,1]]+$

( (Abs [Extract [values, \{Position [values, Nearest [Take[values [ $[A 11,2]]$, $\{$ Position[values, Min[values $[[A 11,2]]]][[1,1]]$, Position [values, Last [values [ [Al1, 2] ] ] [ [1, 1] ] ] , (Mean [values [ [Al1, 2] ] ] 3) ] [ [1] ] [ [1] ] ] [ [1]]] - Abs [Mean[values [ [Al1, 2]]] - 3]) /

(Abs [Extract [values, \{Position[values, Nearest [Take[values [ [Al1, 2]], \{Position [values, $\operatorname{Min}[\operatorname{values}[[\mathrm{Al1}, 2]]]][[1,1]]$, Position [values, Last $[\operatorname{values}[[A 11,2]]]][[1,1]]\}]$, (Mean [values [ [Al1, 2] ] ] - 3)] [[1]]][[1]]\}][[1]]]-

Abs [Extract [values, \{Position[values, Nearest [Take [values [ [Al1, 2]] \{Position[values, $\operatorname{Min}[\operatorname{values}[[\mathrm{All}, 2]]]][[1,1]]$, Position [values, Last [values $[[A 11,2]]]][[1,1]]\}]$, $($ Mean [values [ [All, 2] ] $]-3)][[1]]][[1]]+\{1,0\}\}][[1]]])))]$, (*if not true or false then pull out resonance value*) Extract [

values,

$\{$ ReplacePart [Position [values,

Nearest [Take [values $[$ [All, 2] ], $\{$ Position[values, Min [values $[[A 11,2]]]][[$

$1,1]]$, Position [values, Last [values $[[A 11,2]]]][[1,1]]\}]$,

$($ Mean [values [ [Al1, 2] ] ] - 3) ] [ [1]]] [ [1]], 2 $\rightarrow 1]\}][[1]]])$;

leftvalue $=($ If $[$ Abs $[$ Mean $[$ values $[[$ Al1, 2] $]]-3]>$ Abs [Nearest [

Take [values $[[A 11,2]],\{1, \operatorname{Position}[\operatorname{values,} \operatorname{Min}[\operatorname{values}[[A 11,2]]]][[1,1]]\}]$,

(Mean [values [ [Al1, 2] ] $]-3)]][[1]]$, Interpolation [values [ [All, 1] ],

( ( (Abs [Mean [values [ [All, 2] ] ] - 3] - Abs [Extract [values, \{Position[values, Nearest [ Take $[\operatorname{values}[[A 11,2]],\{1$, Position[values, Min[values $[[A 11,2]]]][[$ $1,1]]\}],($ Mean [values [ [Al1, 2]]] - 3)][[1]]][[1]]\}][[1]]])/

(Abs [Extract [values, \{Position [values, Nearest [Take [values [ [All, 2] ], $\{1, \operatorname{Position}[$ values, $\operatorname{Min}[\operatorname{values}[[\mathrm{Al1}, 2]]]][[1,1]]\}]$, $($ Mean [values [ [Al1, 2] ] $]-3)][[1]]][[1]]+\{1,0\}\}][[1]]]-$

Abs [Extract $[$ values, Position $[$ values, Nearest $[$ Take $[$ values $[[A 11,2]]$,

Figure D.3: Microcoil data processing part (c). 


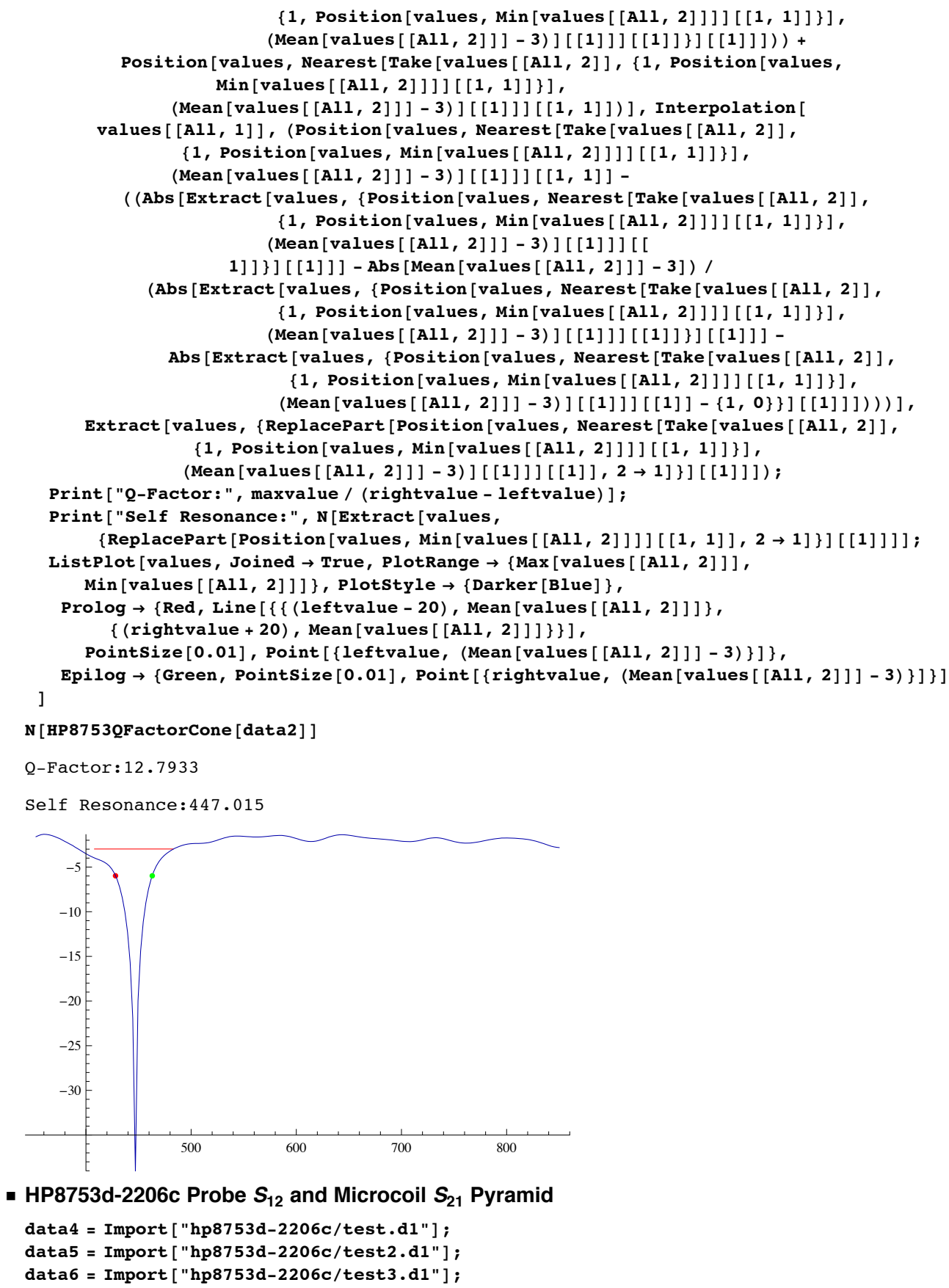

- HP8753d-2206c Probe $S_{12}$ and Microcoil $S_{21}$ Pyramid

data4 = Import $[$ "hp8753d-2206c/test.d1"];

data5 = Import [ hp8753d-2206c/test2.d1"]; data6 $=$ Import [ "hp8753d-2206c/test3.d1"] ;

Figure D.4: Microcoil data processing part (d). 


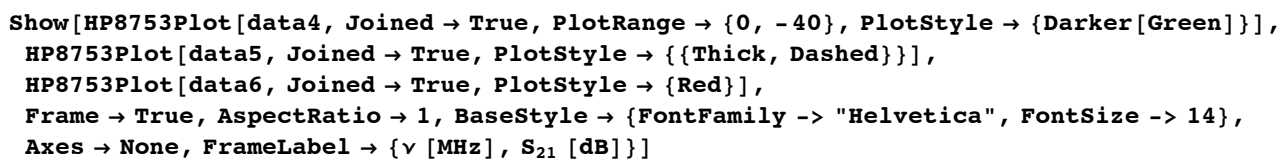

Axes $\rightarrow$ None, FrameLabel $\left.\rightarrow\left\{v[\mathrm{MHz}], \mathbf{S}_{21}[\mathrm{~dB}]\right\}\right]$

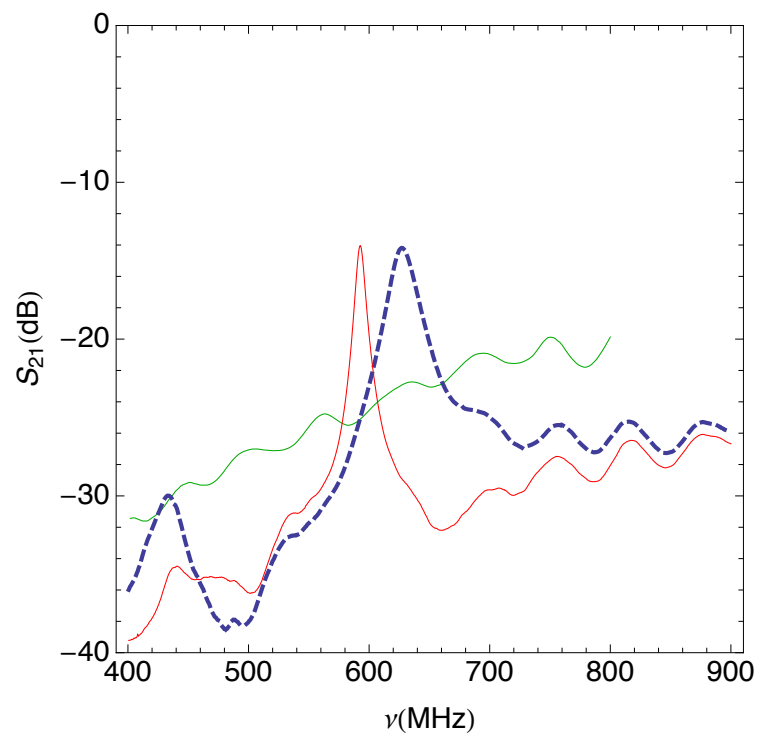

HP8753QFactorPyramid[data_] := Module [\{start, stop, $n$, values, maxvalue

rightvalue, leftvalue, abovemax, belowmax, $m, 0$, downslope, upslope\},

start $=$ ToExpression $[$ StringSplit $[$ data $[[7,1]]][[2]]] / 10^{\wedge} 6$;

stop $=\operatorname{ToExpression}[\operatorname{StringSplit}[\operatorname{data}[[7,1]]][[3]]] / 10^{\wedge} 6$;

$\mathbf{n}=$ ToExpression $[$ StringSplit $[\operatorname{data}[[7,1]]]][[4]]$;

values $=$ Table $[$ start $+\mathbf{k}$ (stop - start $) / \mathbf{n}$,

$\left.\left.\operatorname{data}[[\mathbf{k}+9]] / .\left\{a_{-}, b_{-}\right\} \rightarrow 20 / \log [10] \log [\operatorname{Abs}[a+I b]]\right\},\{k, 1, \mathbf{n}\}\right] ;$

maxvalue = Extract $[$ values, $\{$ ReplacePart [

Position [values, Max [values [[Al1, 2]] ] ] [1 , 1]], 2 $\rightarrow 1]\}][[1]]$;

abovemax $=$ Take $[$ values, $\{$ Position $[$ values, $\operatorname{Max}[$ values $[[A 11,2]]]][1,1]]$,

Position [values, Last[values $[[A 11,2]]]][[1,1]]\}]$;

belowmax $=$ Take $[\operatorname{values},\{1, \operatorname{Position}[\operatorname{values}, \operatorname{Max}[\operatorname{values}[[A 11,2]]]][[1,1]]\}]$;

$\mathrm{m}=1$; While $[\operatorname{abovemax}[[\mathrm{m}, 2]]>\operatorname{abovemax}[[\mathrm{m}+1,2]], \mathrm{m}++]$;

$0=\operatorname{Position}[$ values, $\operatorname{Max}[\operatorname{values}[[A 11,2]]]][[1,1]]$;

While [belowmax $[0,2]]>$ belowmax $[[0-1,2]], 0--]$;

downslope $=$ Take $[$ abovemax, $\{1, \mathrm{~m}\}]$;

upslope $=$ Take $[$ belowmax, $\{0, \operatorname{Position}[\operatorname{values}, \operatorname{Max}[\operatorname{values}[[\mathrm{All}, 2]]]][[1,1]]\}]$;

rightvalue $=($ If $[(\operatorname{Max}[\operatorname{values}[[\mathrm{Al1}, 2]]]-3)>$

Nearest [downslope $[[A 11,2]],(\operatorname{Max}[\operatorname{values}[[A 11,2]]]-3)][[1]]$,

Interpolation [downslope $[[A 11,1]]$, (Position [downslope,

Nearest [downslope $[[A 11,2]],(\operatorname{Max}[\operatorname{values}[[A 11,2]]]-3)][[1]]][[1,1]]-$

$(((\operatorname{Max}[\operatorname{values}[[A 1), 2]]]-3)-$ Extract [downslope, \{Position [downslope, Nearest [ downs lope [[Al1, 2]], (Max[values [ [Al1, 2] ] - 3)] [ [1]]][[1]]\}] [ [1]]) /

(Extract [downslope, \{Position [downslope, Nearest [downslope $[[A 11,2]]$,

$(\operatorname{Max}[\operatorname{values}[[\mathrm{Al1}, 2]]]-3)][[1]]][[1]]-\{1,0\}\}][[1]]-$

Extract [downslope, \{Position [downslope, Nearest [downslope $[$ [All, 2]] $(\operatorname{Max}[\operatorname{values}[[\mathrm{All}, 2]]]-3)][[1]]][[1]]\}][[1]])))]$,

Interpolation [downslope $[$ [All, 1]] , (Position[downslope, Nearest [

downslope $[[A 11,2]],(\operatorname{Max}[\operatorname{values}[[\mathrm{Al1}, 2]]]-3)][[1]]][[1,1]]+$

( (Extract [downslope, \{Position [downslope, Nearest [downslope [ [Al1, 2]],

FiguRE D.5: Microcoil data processing part (e). 


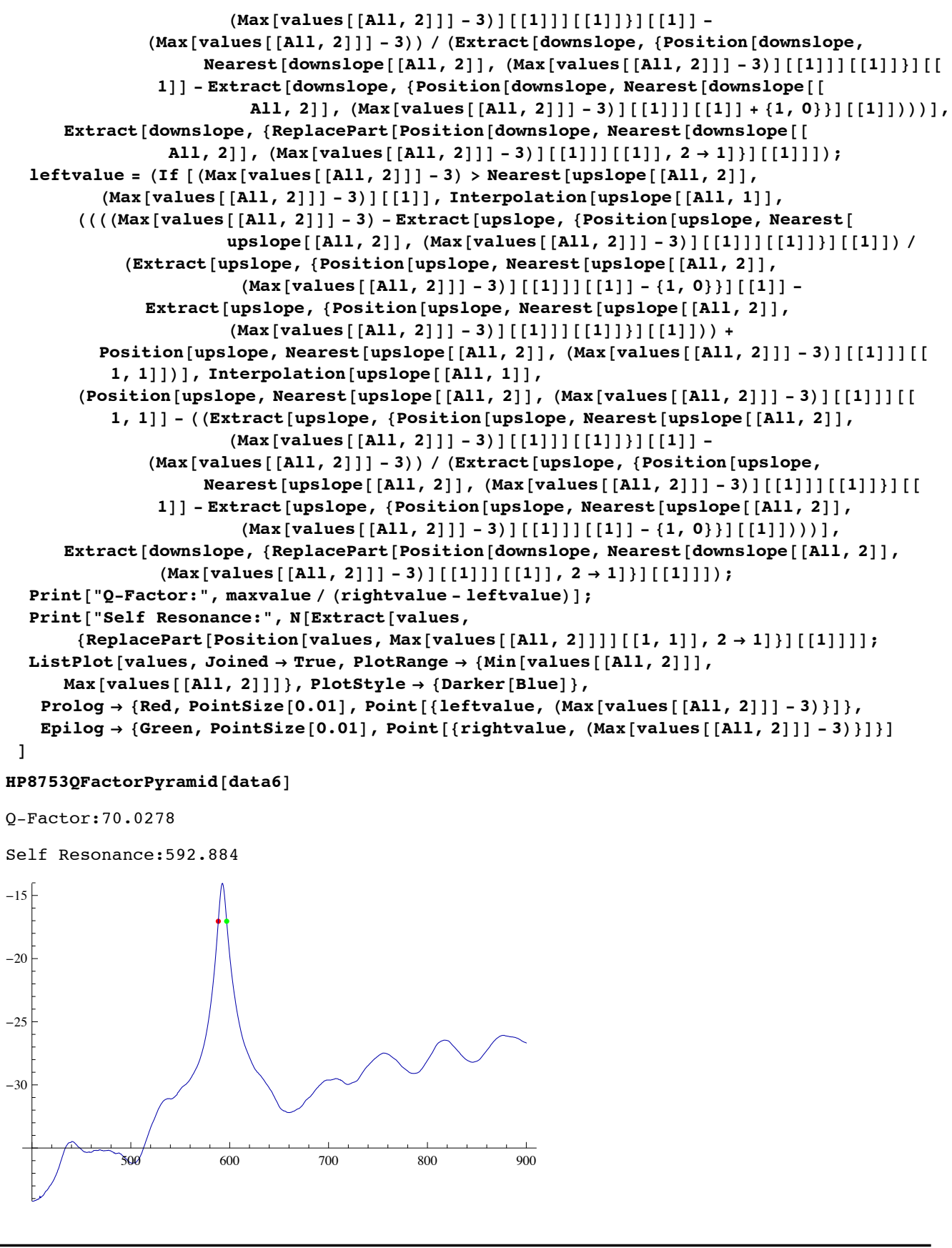

Figure D.6: Microcoil data processing part (f). 


\section{- Alternate Calculation}

HP87530FactorPyramid[data_] : =

Module [ start, stop, $n$, values, maxvalue, rightvalue, leftvalue,

reverseinput, abovemax, belowmax, $m, 0$, downslope, upslope

start $=$ ToExpression $[$ StringSplit $[\operatorname{data}[[7,1]]][[2]]] / 10^{\wedge} 6$;

stop $=$ ToExpression $[$ StringSplit $[\operatorname{data}[[7,1]]][[3]]] / 10^{\wedge} 6$;

n = ToExpression [StringSplit $[\operatorname{data}[[7,1]]]][[4]] ;$

values $=$ Table $[$ s start $+\mathbf{k}$ (stop - start $) / n$,

$\left.\left.\operatorname{data}[[\mathbf{k}+9]] / .\left\{a_{-}, b_{-}\right\} \rightarrow 20 / \log [10] \log [\operatorname{Abs}[a+I b]]\right\},\{k, 1, \mathbf{n}\}\right]$;

maxvalue = Extract $[$ values, $\{$ ReplacePart [

Position [values, Max [values [ [Al1, 2] ] ] [ [1, 1]], 2 $\rightarrow 1]\}][[1]]$;

reverseinput $[$ input $]:=N[$ Table $[\{$ input $[[1+i, 2]]$, input $[[1+i, 1]]\}$

$\{i, 0$, (Position [input, Last [input] $][[1,1]]-1)\}]]$;

abovemax $=$ Take $[$ values, $\{$ Position $[$ values, $\operatorname{Max}[$ values $[[A 11,2]]]][[1,1]]$,

Position [values, Last[values [ [Al1, 2] ] ] $[[1,1]]\}]$;

belowmax $=$ Take $[\operatorname{values},\{1, \operatorname{Position}[\operatorname{values}, \operatorname{Max}[\operatorname{values}[[A 11,2]]]][[1,1]]\}]$;

$\mathrm{m}=1$; While $[$ abovemax $[[\mathrm{m}, 2]]>\operatorname{abovemax}[[\mathrm{m}+1,2]], \mathrm{m}++]$;

$0=\operatorname{Position}[$ values, $\operatorname{Max}[\operatorname{values}[[A 11,2]]]][[1,1]]$;

While $[$ belowmax $[[0,2]]>\operatorname{belowmax}[[0-1,2]], 0--]$;

downslope $=$ Take $[$ abovemax, $\{1, \mathrm{~m}\}]$

upslope $=$ Take $[$ belowmax,$\{0, \operatorname{Position}[\operatorname{values}, \operatorname{Max}[\operatorname{values}[[\mathrm{All}, 2]]]][1,1]]\}]$;

rightvalue $=$ Interpolation $[$ reverseinput $[$ downslope $],(\operatorname{Max}[\operatorname{values}[[A 11,2]]]-3)]$

leftvalue = Interpolation [reverseinput [upslope], (Max [values $[$ [All, 2] ] ] - 3) ];

Print ["Q-Factor: ", maxvalue / (rightvalue- leftvalue)] ;

Print ["Self Resonance:", N[Extract [values,

$\{$ ReplacePart [Position [values, Max[values[[Al1, 2] ]] $[[1,1]], 2 \rightarrow 1]\}][[1]]]]$;

ListPlot [values, Joined $\rightarrow$ True, PlotRange $\rightarrow$ \{Min[values [ $[A l 1,2]]]$,

Max $[$ values $[$ [Al1, 2] ] ] $\}$, PlotStyle $\rightarrow\{$ Darker [Blue $]\}$,

Prolog $\rightarrow\{$ Red, PointSize [0.01], Point [\{leftvalue, (Max[values[[Al1, 2] ] $]-3)\}]\}$,

Epilog $\rightarrow\{$ Green, PointSize[0.01], Point [\{rightvalue, (Max[values [All, 2] ] $]-3)\}]\}]$ ]

HP8753QFactorPyramid [data6]

Q-Factor: 70.0395

Self Resonance:592.884

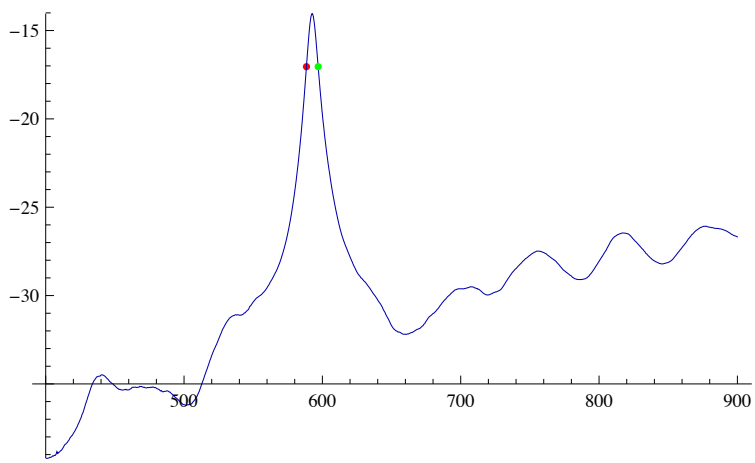

Figure D.7: Microcoil data processing part (g). 
Appendix E

Mathematica FID and Imaging

Data Processing 


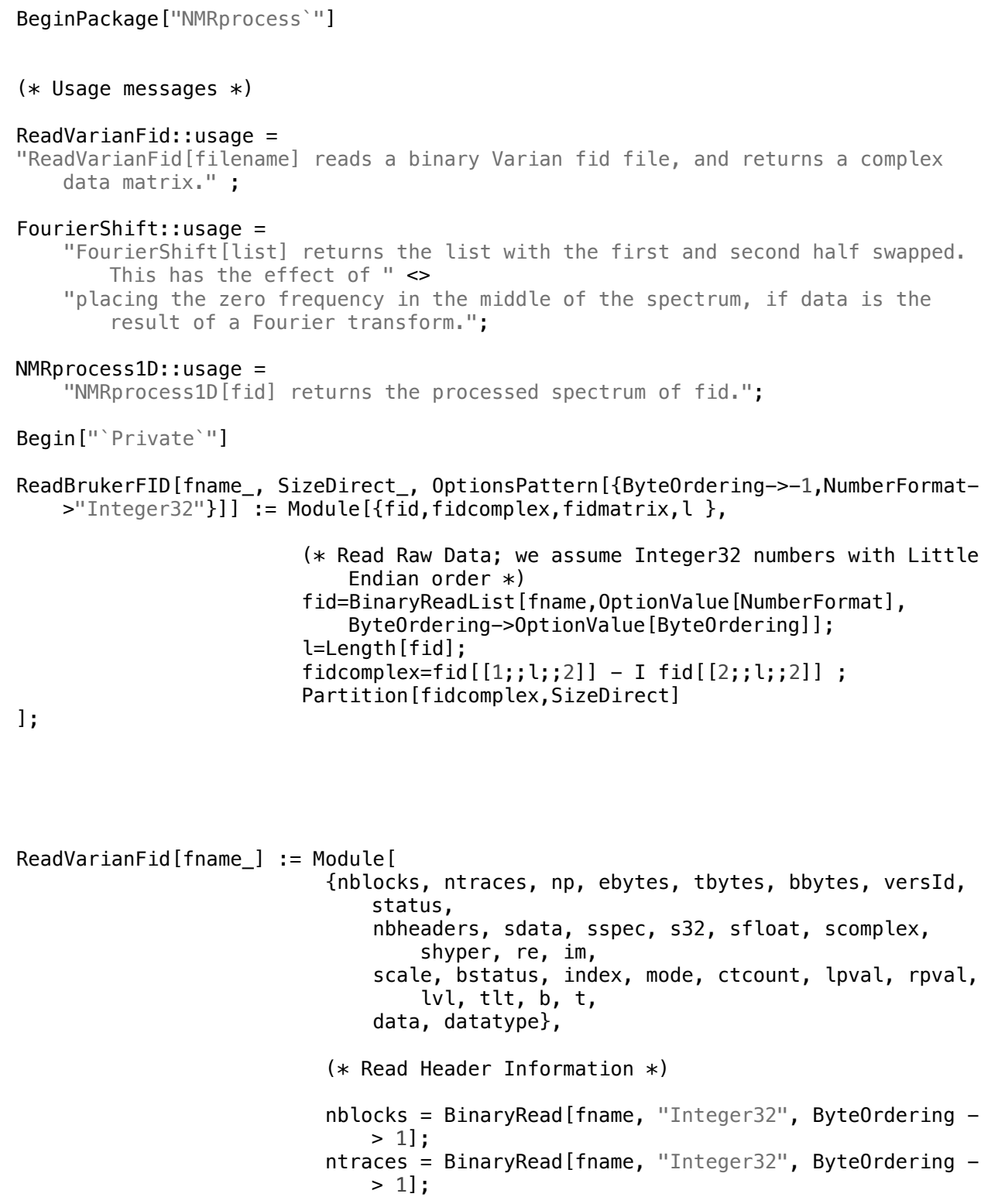

FIGURE E.1: Varian data import routine part (a). 
$\mathrm{np}=$ BinaryRead[fname, "Integer32", Byte0rdering $\rightarrow$ 1]; ebytes = BinaryRead [fname, "Integer32", Byte0rdering $\rightarrow$ $1]$;

tbytes = BinaryRead[fname, "Integer32", Byte0rdering $\rightarrow$ 1];

bbytes = BinaryRead[fname, "Integer32", Byte0rdering $\rightarrow$ $1]$;

versId = BinaryRead[fname, "Integer16", Byte0rdering $\rightarrow$ $1]$;

status = BinaryRead [fname, "Integer16", Byte0rdering $\rightarrow$ 1];

nbheaders = BinaryRead[fname, "Integer32", Byte0rdering $\rightarrow 1]$;

sdata $=$ BitGet [status, 0] == 1 ;

sspec = BitGet [status, 1] == 1

s32 = BitGet [status, 2] == 1;

sfloat $=$ BitGet $[$ status, 3] == 1;

scomplex = BitGet [status, 4] == 1;

shyper $=$ BitGet $[$ status, 5$]==1$;

datatype = "Integer16";

If $[$ s32, datatype = "Integer32"] ;

If [sfloat, datatype = "Real32"];

Print ["Reading ",nblocks, " blocks of ", $n p / 2$, "

complex points; data type: ", datatype];

(* Print [datatype] ; *)

(* Read Blocks *)

re $=\{\} ;$

Do [

(* Print ["Reading block ",b]; *)

(* Read block header *)

scale = BinaryRead [fname, "Integer16", Byte0rdering $\rightarrow 1]$;

bstatus = BinaryRead [fname, "Integer16", Byte0rdering $\rightarrow 1]$;

index = BinaryRead [fname, "Integer16", Byte0rdering

$$
\rightarrow 1] \text {; }
$$

mode $=$ BinaryRead $[$ fname, "Integer16", Byte0rdering $>1]$;

ctcount = BinaryRead [fname, "Integer32", Byte0rdering $\rightarrow 1]$;

lpval = BinaryRead [fname, "Real32", Byte0rdering $\rightarrow$ $1]$;

rpval = BinaryRead [fname, "Real32", Byte0rdering $\rightarrow$ $1]$;

lvl = BinaryRead [fname, "Real32", Byte0rdering $\rightarrow 1]$

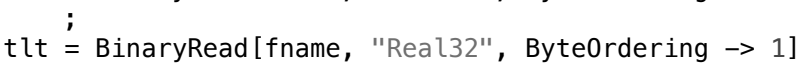

Do [

data $=$

Figure E.2: Varian data import routine part (b). 


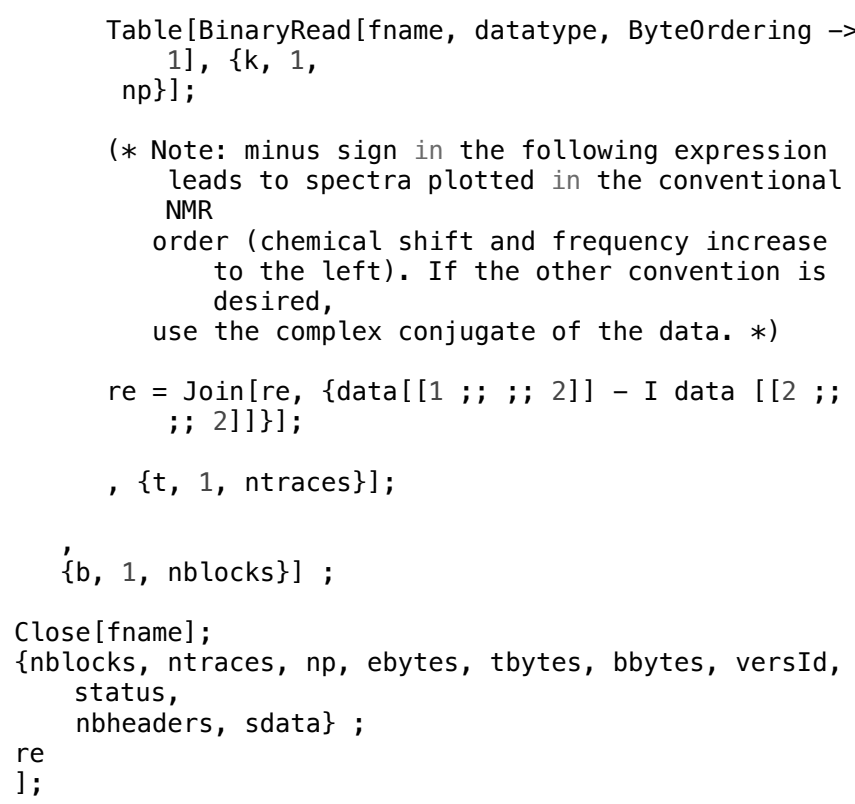

SetAttributes [ReadVarianFid, Join [Attributes [ReadVarianFid] , \{ReadProtected\}] ] ;

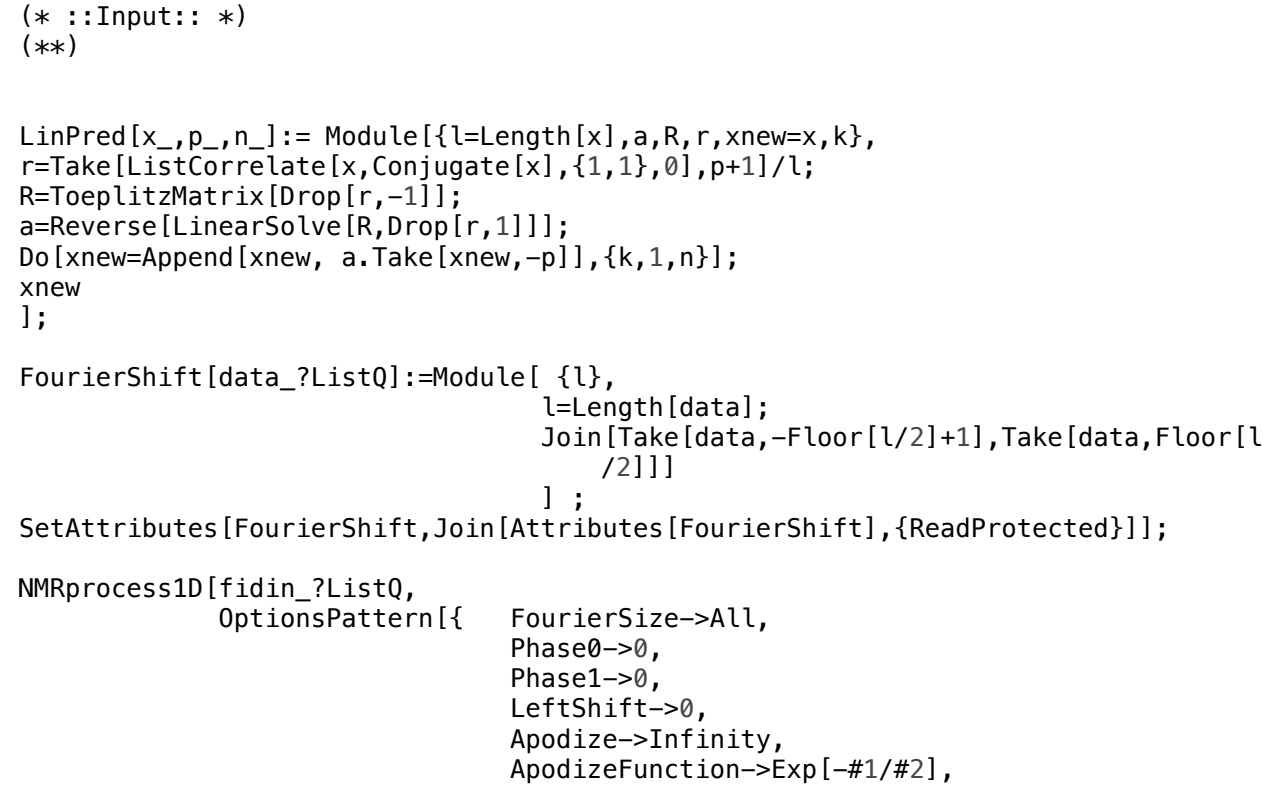

FiguRE E.3: Varian data import routine part (c). 


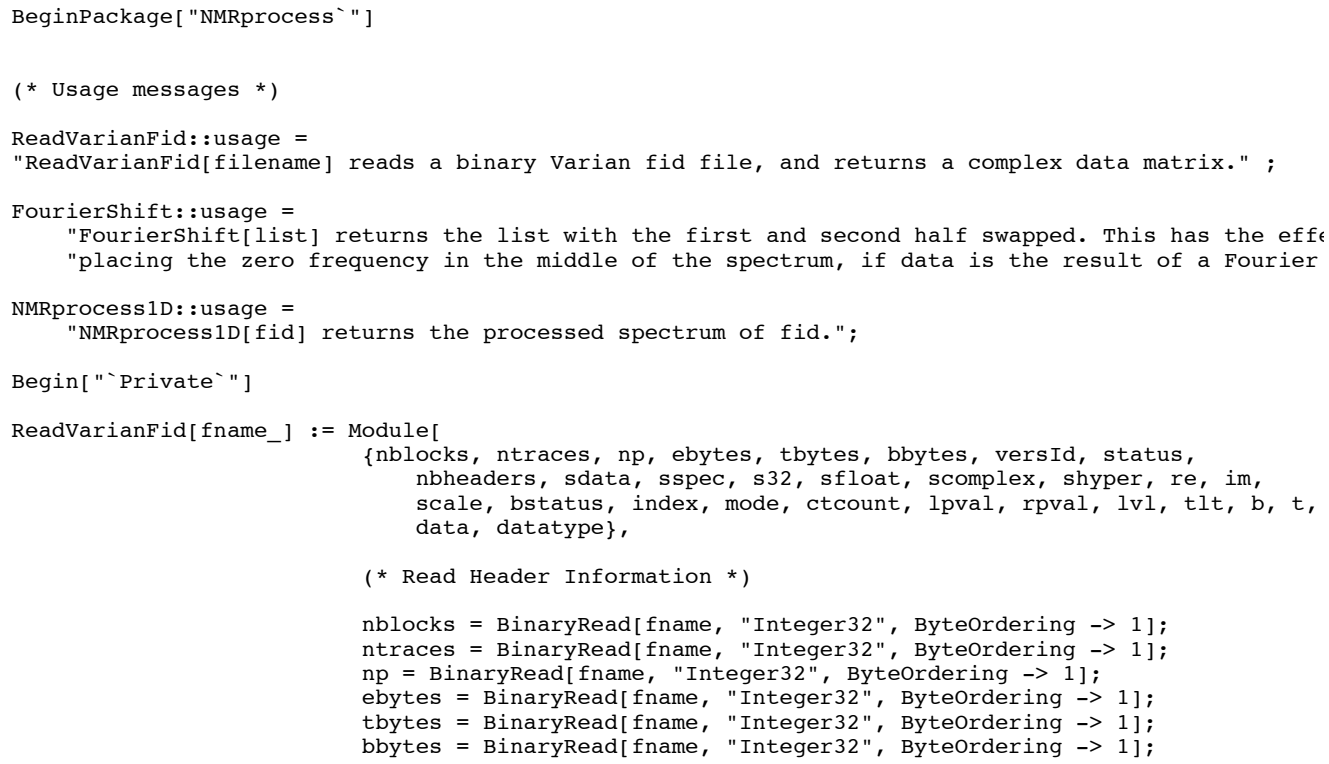

FiguRE E.5: Mathematica spectra processing routine part (a). 


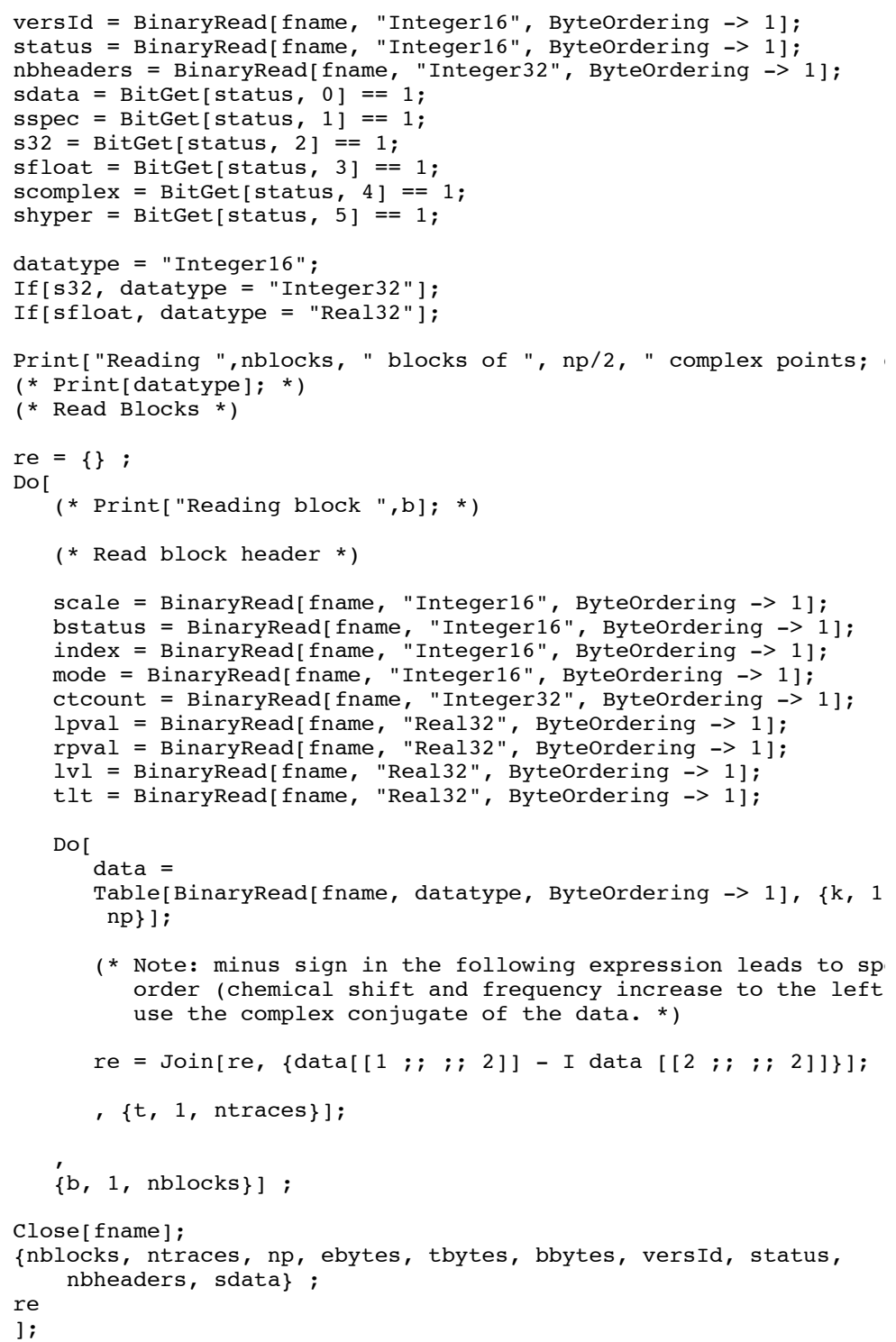

SetAttributes [ReadVarianFid, Join [Attributes [ReadVarianFid], \{ReadProtected\}] ] ;

FourierShift[data_?ListQ]:=Module[ $\{1\}$,

l=Length [ data ] ;

Join [Take [data, $-F l o o r[1 / 2]+1]$, Take [data,Floor[1/2]]]

] ;

SetAttributes [FourierShift, Join [Attributes [FourierShift], \{ReadProtected\}] ] ;

NMRprocess1D[fidin_?ListQ,

OptionsPattern[ $\{$ FourierSize->All,

Phase0 $->0$,

Phase1->0,

FiguRE E.6: Mathematica spectra processing routine part (b). 


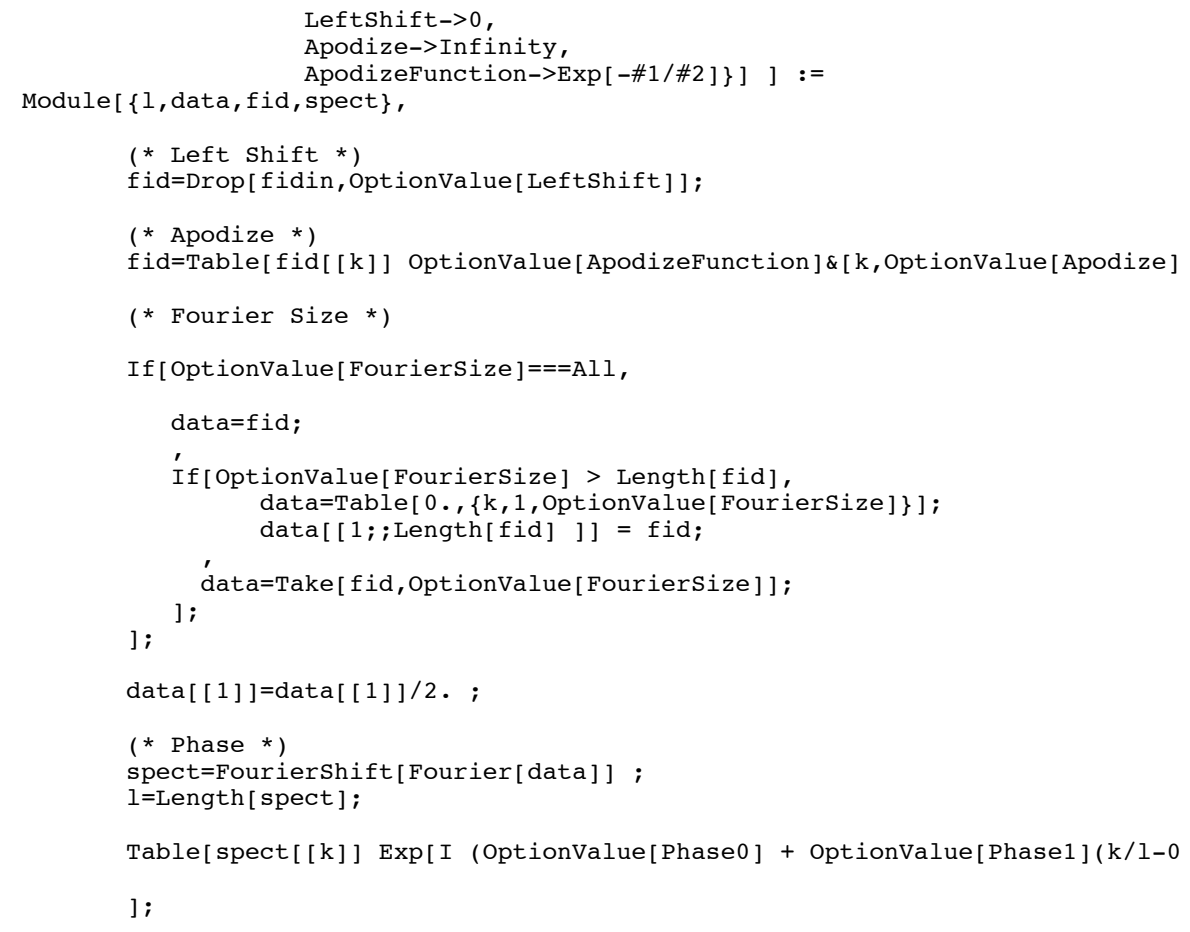

End [ ]

EndPackage [ ]

\section{Reading the FID and Processing}

- Reading Varian binary formatted FID files:

data = ReadVarianFid ["spect-2292b.fid"];

Reading 1 blocks of 10000 complex points; data type: Real32

nblocks $=1$;

cpoints $=10000 ;$

spectrawidth $=5000$;

\section{- Phasing a spectrum}

The data above contains 16000 complex points for each block. We can Fourier transform it at low resolution at first, to obtain values for the zero and first order phase correction. Then these values can be used to generate a high-resolution plot.

FIGURE E.7: Mathematica spectra processing routine part (c). 
Manipulate [ListPlot [Re [NMRprocess1D [data [ [1] ], Phase0 $\rightarrow$ ph0 $\pi$,

Phase $1 \rightarrow \operatorname{ph} 1 \pi$, LeftShift $\rightarrow 0$, FourierSize $\rightarrow$ cpoints ] ], PlotJoined $\rightarrow$ True

PlotRange $\rightarrow\{$ All, All $\}$, Frame $\rightarrow$ True $],\{\{$ ph0, 0$\},-1,1\},\{\{$ ph 1,0$\},-10,10\}]$

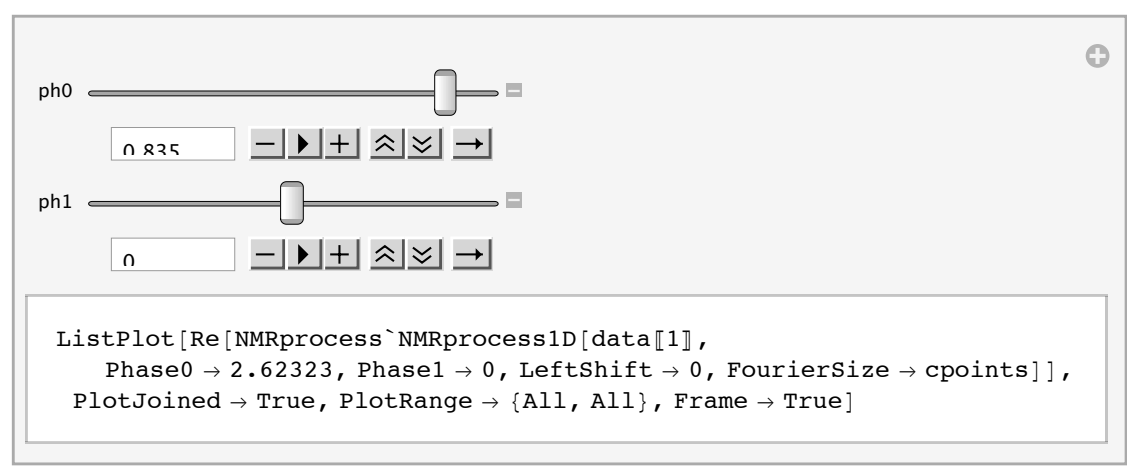

pha0 $=.835 ;$

pha1 =0;

apodize $=8000$;

leftshift $=0$;

nutationdata $=$ Table $[\operatorname{Re}[$ NMRprocess $1 \mathrm{D}[$ data $[[\mathrm{k}]]$

Phase $\rightarrow$ pha0 $\pi$, Phase $\rightarrow$ pha1 $\pi$, LeftShift $\rightarrow$ leftshift, Apodize $\rightarrow$ apodize,

FourierSize $\rightarrow$ cpoints, ApodizeFunction $\rightarrow \operatorname{Exp}[-(\# 1 / \# 2)]]],\{\mathbf{k}, 1$, nblocks $\}]$;

maxblock $=$ Position $[$ nutationdata, Max $[$ nutationdata $]][[1,1]]$;

norm $=$ StandardDeviation $[$ Take $[\operatorname{Re}[$

NMRprocess1D[data [ [maxblock] ], Phase $\rightarrow$ pha0 $\pi$, Phase1 $\rightarrow$ pha1 $\pi$, LeftShift $\rightarrow$ leftshift, Apodize $\rightarrow$ apodize, FourierSize $\rightarrow$ cpoints, ApodizeFunction $\rightarrow \operatorname{Exp}[-(\# 1 / \# 2)]]], 500]]$;

NMRPlot [fid_, ctr_, scale_, pha0_, pha1_, leftshift_, apodize_, cpoints_, nblocks_,

spectrawidth_] := Module[\{nutationdata, maxblock, norm, datafid, values\},

nutationdata $=$ Table $[\operatorname{Re}[$ NMRprocess1D $[\mathrm{fid}[\mathrm{k}]]$, Phase $\rightarrow$ pha0 $\pi$, Phase $1 \rightarrow$ pha1 $\pi$, LeftShift $\rightarrow$ leftshift, Apodize $\rightarrow$ apodize, Fouriersize $\rightarrow$ cpoints,

ApodizeFunction $\rightarrow \operatorname{Exp}[-(\# 1 / \# 2)]]],\{k, 1$, nblocks $\}]$;

maxblock $=$ Position $[$ nutationdata, $\operatorname{Max}[$ nutationdata $][[1,1]]$;

norm =

StandardDeviation [Take [Re [NMRprocess1D [fid[ [maxblock] ], Phase0 $\rightarrow$ pha0 $\pi$, Phase1 $\rightarrow$ pha1 $\pi$ LeftShift $\rightarrow$ leftshift, Apodize $\rightarrow$ apodize, FourierSize $\rightarrow$ cpoints, ApodizeFunction $\rightarrow \operatorname{Exp}[-(\# 1 / \# 2)]]], 500]]$;

datafid $=\operatorname{Re}[$ NMRprocess $1 D[$ data $[$ maxblock] $]$, Phase $\rightarrow$ pha0 $\pi$,

Phase $1 \rightarrow$ phal $\pi$, LeftShift $\rightarrow$ leftshift, Apodize $\rightarrow$ apodize,

FourierSize $\rightarrow$ cpoints, ApodizeFunction $\rightarrow \operatorname{Exp}[-(\# 1 / \# 2)]]] /$ norm;

values $=\operatorname{Table}\left[\left\{\mathrm{N}\left[\frac{\operatorname{spectrawidth}(i-1)}{\operatorname{cpoints} 600}-\operatorname{ctr}\right]\right.\right.$, datafid $\left.\left.[i]\right]\right\},\{i, 1$, Length $[$ datafid] $\}] ;$

ListPlot [values, PlotJoined $\rightarrow$ True, Axes $\rightarrow$ \{True, False $\},$ PlotRange $\rightarrow$ scale $\{-0.1,0.9\}$,

PlotLabel $\rightarrow$ Style $\left["{ }^{1} \mathrm{H}\right.$ NMR of $5 \% \mathrm{H}_{2} \mathrm{O}$ in $\mathrm{D}_{2} \mathrm{O} ", 17$, Bold, Red, TextAlignment $\rightarrow$ Center],

AxesLabel $\rightarrow\{$ "ppm", " $\}$,

Basestyle $\rightarrow$ FontFamily $\rightarrow$ "Courier", Bold, 12\}, Imagesize $\rightarrow 600]$

]

FIGURE E.8: Mathematica spectra processing routine part (d). 
NMRPlot $[$ data $, 3,1500, .835,0,0,8000,10000,1,5000]$

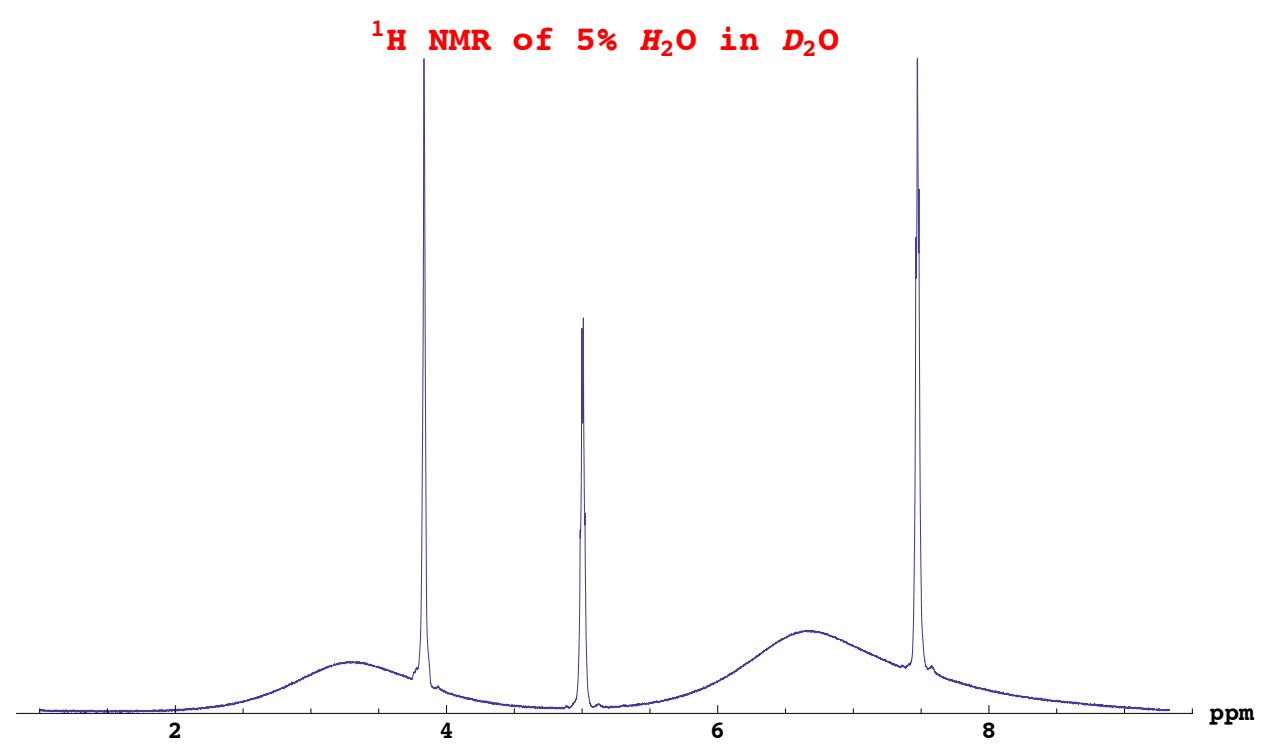

\section{ListPlot [}

Re [NMRprocess1D [data [ [maxblock] ], Phase $\rightarrow$ pha0 $\pi$, Phase $1 \rightarrow$ pha1 $\pi$, LeftShift $\rightarrow$ leftshift, Apodize $\rightarrow$ apodize, FourierSize $\rightarrow$ cpoints, ApodizeFunction $\rightarrow \operatorname{Exp}[-(\# 1 / \# 2)]]] /$ norm, DataRange $\rightarrow\{-6,0\}$, PlotJoined $\rightarrow$ True, PlotRange $\rightarrow\{$ Full, Full $\}$, Axes $\rightarrow\{$ False, False $\}$, Frame $\rightarrow$ True, FrameTicks $\rightarrow$ \{Automatic, Automatic, True, True ,

Framelabel $\rightarrow\{$ Style [" $\delta(\mathrm{ppm}) ", 15$, Bold], Style ["SNR", 15, Bold], None, None $]$

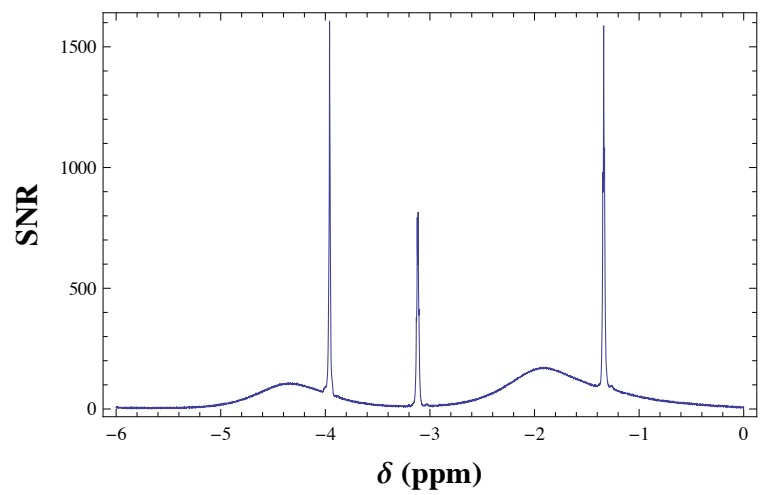

(*Export [ "spect-0xxx.pdf",\%]*)

FIGURE E.9: Mathematica spectra processing routine part (e). 


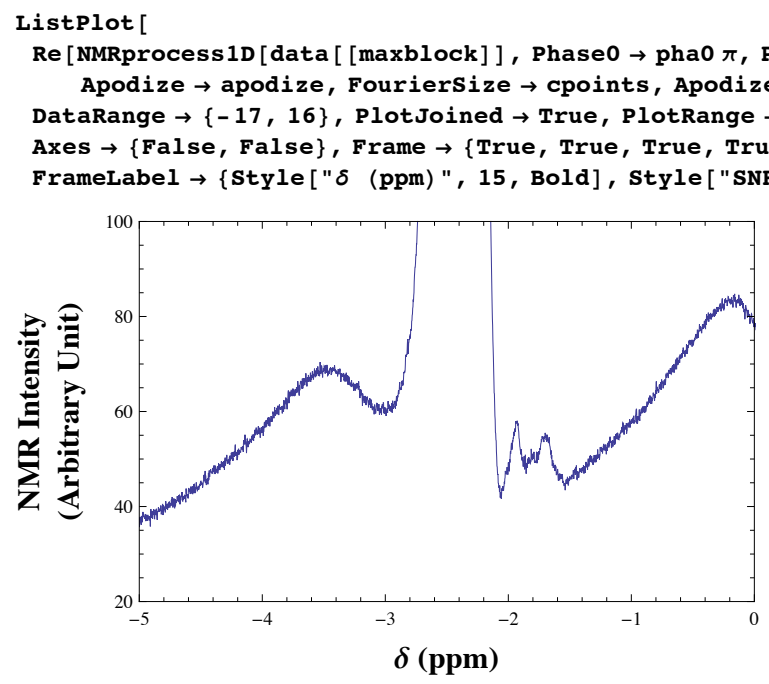

(*Export [ "spect-0xxx-1ineshape.pdf",\%]*)

Show [GraphicsArray [Table [ListPlot [

Re [NMRprocess1D [data [ [k] ], Phase $\rightarrow$ pha0 $\pi$, Phase1 $\rightarrow$ pha1 $\pi$, LeftShift $\rightarrow$ leftshift,

Apodize $\rightarrow$ apodize, FourierSize $\rightarrow$ cpoints, ApodizeFunction $\rightarrow \operatorname{Exp}[-(\# 1 / \# 2)]]] /$ norm,

PlotJoined $\rightarrow$ True, DataRange $\rightarrow\{-17,16\}$, PlotRange $\rightarrow\{$ Full, $\{-22000,28000\}\}$,

Axes $\rightarrow$ False, AspectRatio $\rightarrow 30],\{k, 1$, nblocks $\}]\}$, PlotRangePadding $\rightarrow 500$

Frame $\rightarrow$ True, FrameTicks $\rightarrow\{$ Automatic, True $\},\{$ True, True $\}$,

Framelabel $\rightarrow$ \{Style ["Pulse Length $(\mu s) ", 15$, Bold], Style ["NMR Intensity

(Arbitrary Unit) ", 15, Bold], None, None ] ]

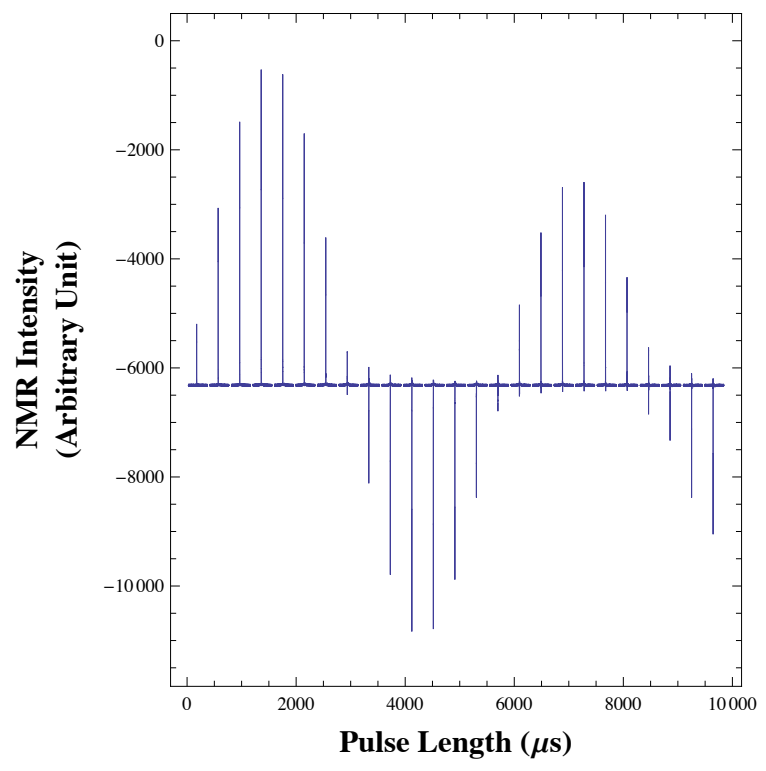

(*Export [ "nutation_0xxx.pdf",\%] *)

Figure E.10: Mathematica spectra processing routine part (f). 


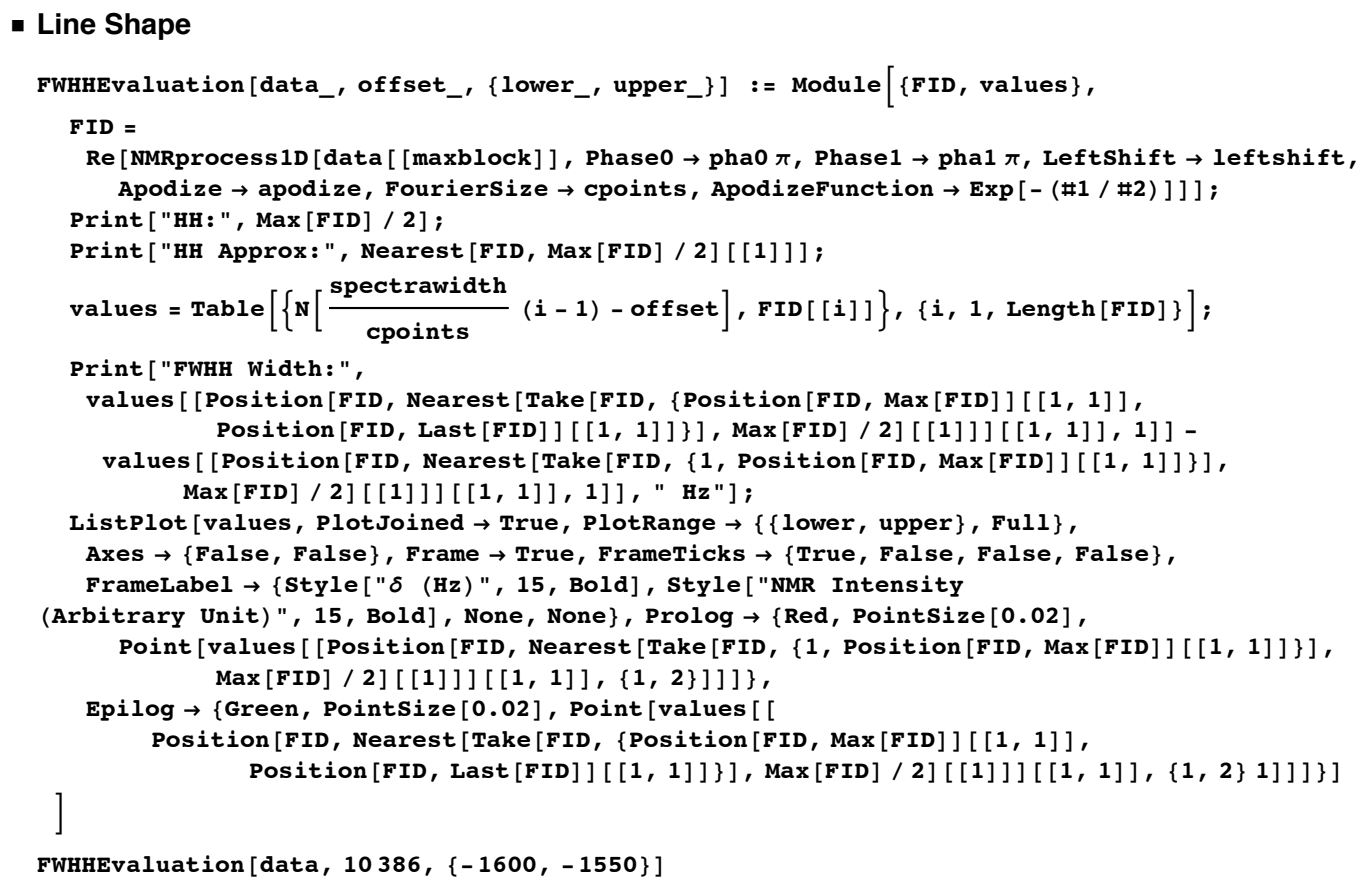

$\mathrm{HH}: 2424.77$

HH Approx:2418.29

FWHH Width: $8.82044 \mathrm{~Hz}$

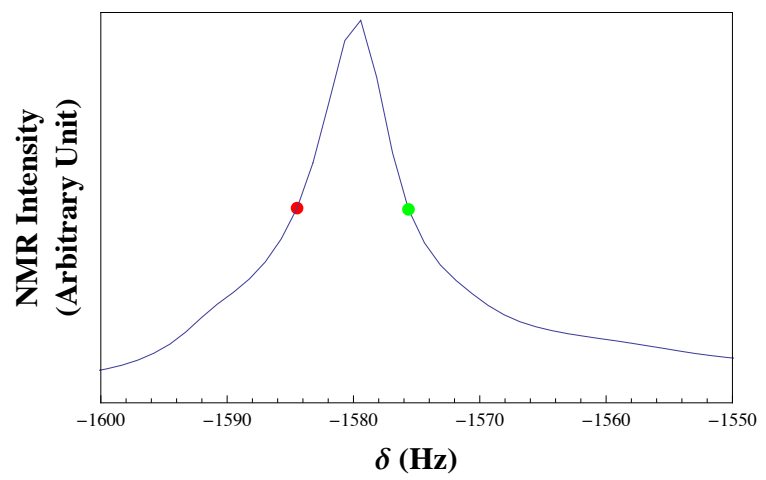

\section{- Probe Efficiency}

Input the power in $\mathrm{dB}$ and starting value/interval used for nutation.

pwrdB $=56$;

pwrWatt $=$ Solve $\left[\operatorname{pwrdB}=10 \log \left[10, \frac{\mathrm{W}}{(0.001)}\right], \mathrm{W}\right][[1]][[1]]$

$\mathrm{W} \rightarrow 398.107$

FIGURE E.11: Mathematica spectra processing routine part (g). 
start $=1$;

interval $=2$;

(*entered above

nutationdata $=$

Table [Re [NMRprocess1D [data [ [k] ], Phase $\rightarrow$ pha0 $\pi$, Phase $1 \rightarrow$ pha1 $\pi$, LeftShift $\rightarrow 0$, Apodize $\rightarrow 8000$ FourierSize $\rightarrow$ cpoints, ApodizeFunction $\rightarrow \operatorname{Exp}[-(\# 1 / \# 2)]]],\{k, 1$, nblocks $\}] ; *)$

totaltime $=($ interval $($ Position [nutationdata, Max [nutationdata] $][1,1]])-$ start) 4

28

Print ["Probe Efficiency:", $\frac{1}{\left(\frac{\text { totaltime }}{1000^{2}} \text { Sqrt [W/. pwrWatt] }\right)} \frac{1}{1000}$ ]

Probe Efficiency: 1.78995

\section{- Specific Sensitivity}

- Example

Example 1: $\mathrm{H}_{2} O \rightarrow \frac{1 \mathrm{~mL}}{1 g}$, so ' $\mathrm{X}$ ' $\mu \mathrm{L}$ or $\mathrm{H}_{2} O$ is:

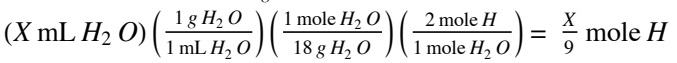

Example 2: $200 \mathrm{mg}$ Glucose $\rightarrow C_{6} H_{12} O_{6}$ in $2000 \mu \mathrm{L} D_{2} O$ where $D_{2} O \rightarrow 1.107 \frac{\mathrm{g}}{\mathrm{cm}^{3}}=$

$1.107 \frac{\mathrm{g}}{\mathrm{mL}}$ and $\mathrm{C}_{6} \mathrm{H}_{12} \mathrm{O}_{6} \rightarrow 1.54 \frac{\mathrm{g}}{\mathrm{cm}^{3}}=1.54 \frac{\mathrm{g}}{\mathrm{mL}}$

$(10 \mu \mathrm{L}$ Sample $)\left(\frac{(0.2 g \text { Glucose })}{(0.2 g \text { Glucose })\left(\frac{1000 \mu \mathrm{L}}{1.54 g}\right)+\left(2000 \mu \mathrm{L} D_{2} O\right)}\right)=0.000939024 g$ Glucose

$(0.000939024 \mathrm{~g}$ Glucose $)\left(\frac{1 \text { mole Glucose }}{180 g \text { Glucose }}\right)\left(\frac{12 \text { mole } H}{1 \text { mole Glucose }}\right)\left(1000^{2} \frac{\mu \text { mole }}{\text { mole }}\right)=62.602 \mu$ mole $H$

- Process

mLSample $=10 / 1000$

$\mu$ MolProtons $=\mathrm{N}\left[\mathrm{mLSample}\left(\frac{1}{18}\right)(2) *\left(1000^{2}\right)\right]$

1111.11

Print ["S/N:", Max [

Re [NMRprocess1D[data[[maxblock] ], Phase0 $\rightarrow$ pha0 $\pi$, Phase $1 \rightarrow$ pha1 $\pi$, LeftShift $\rightarrow$ leftshift Apodize $\rightarrow$ apodize, FourierSize $\rightarrow$ cpoints, ApodizeFunction $\rightarrow \operatorname{Exp}[-(\# 1 / \# 2)]]]] /$

StandardDeviation [Take [Re [NMRprocess1D [data [ [maxblock] ], Phase0 $\rightarrow$ pha0 $\pi$, Phase $1 \rightarrow$ phal $\pi$, LeftShift $\rightarrow$ leftshift, Apodize $\rightarrow$ apodize,

FourierSize $\rightarrow$ cpoints, ApodizeFunction $\rightarrow \operatorname{Exp}[-(\# 1 / \# 2)]]],-500]]]$

$\mathrm{S} / \mathrm{N}: 22551.2$

Print ["Specific Sensitivity:",

$\operatorname{Max}[\operatorname{Re}[$ NMRprocess1D [data [ [maxblock] ], Phase0 $\rightarrow$ pha0 $\pi$, Phase $\rightarrow$ pha1 $\pi$, LeftShift $\rightarrow$ leftshift, Apodize $\rightarrow$ apodize,

FourierSize $\rightarrow$ cpoints, ApodizeFunction $\rightarrow \operatorname{Exp}[-(\# 1 / \# 2)]]]] /$

StandardDeviation [Take [Re [NMRprocess1D [data [ [maxblock] ], Phase0 $\rightarrow$ pha0 $\pi$, Phase $1 \rightarrow$ pha1 $\pi$, LeftShift $\rightarrow$ leftshift, Apodize $\rightarrow$ apodize, FourierSize $\rightarrow$ cpoints ApodizeFunction $\rightarrow \operatorname{Exp}[-(\# 1 / \# 2)]]],-500]] / \mu$ MolProtons $]$

Specific Sensitivity:20.2961

FigURE E.12: Mathematica spectra processing routine part (h). 


\section{Prelims}

SetDirectory [ "/Users/mu3q/Experiment "]

/Users/mu3q/Experiment

$<$ NMRprocess

BaseDirectory = "/Users/mu3q/Experiment/field-map-jan-5-2012/" ;

SetDirectory [BaseDirectory]

/Users/mu3q/Experiment/field-map-jan-5-2012

\section{Process a Varian GEMS Image}

data = ReadVarianFid ["image-0324a.fid/fid"];

Reading 1 blocks of 128 complex points; data type: Real32

xf1=FourierShift/@Fourier/@data ;

xf2=FourierShift /@Fourier /@Transpose $[\mathrm{xf1}]$;

Image $[$ Abs $[x f 2] / 50]$

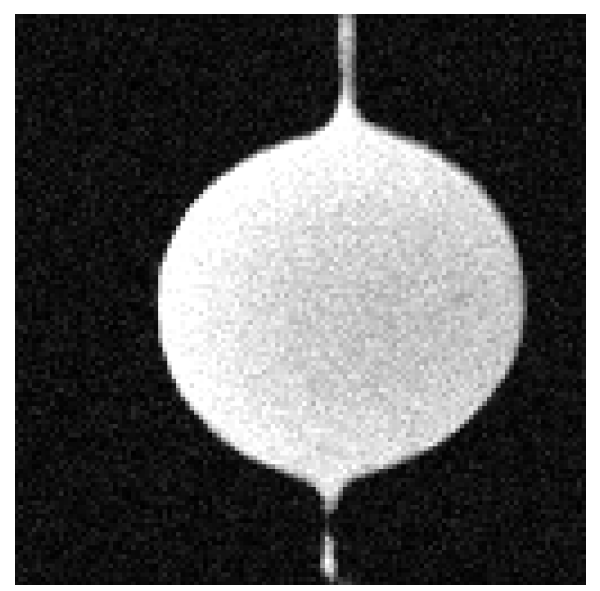

Figure E.13: Mathematica image processing routine. 


\section{Bibliography}

[1] Andréas Manz, N Graber, and HM Widmer. Miniaturized total chemical analysis systems: a novel concept for chemical sensing. Sensors and actuators B: Chemical, 1(1):244-248, 1990.

[2] Nian Wu, Andrew Webb, Timothy L Peck, and Jonathan V Sweedler. Online nmr detection of amino acids and peptides in microbore lc. Analytical chemistry, 67 (18):3101-3107, 1995.

[3] AG Webb. Nuclear magnetic resonance coupled microseparations. Magnetic Resonance in Chemistry, 43(9):688-696, 2005.

[4] Christian Hilty, Erin E McDonnell, Josef Granwehr, Kimberly L Pierce, Song-I Han, and Alexander Pines. Microfluidic gas-flow profiling using remote-detection nmr. Proceedings of the National Academy of Sciences of the United States of America, 102(42):14960-14963, 2005.

[5] Vikram S Bajaj, Jeffrey Paulsen, Elad Harel, and Alexander Pines. Zooming in on microscopic flow by remotely detected mri. Science, 330(6007):1078-1081, 2010.

[6] M Mohmmadzadeh, N Baxan, V Badilita, K Kratt, H Weber, JG Korvink, U Wallrabe, J Hennig, and D Von Elverfeldt. Characterization of a 3d mems 
fabricated micro-solenoid at 9.4 t. Journal of Magnetic Resonance, 208(1):20-26, 2011.

[7] C Massin, G Boero, F Vincent, J Abenhaim, P-A Besse, and RS Popovic. High-i i, qi/i; factor rf planar microcoils for micro-scale nmr spectroscopy. Sensors and Actuators A: Physical, 97:280-288, 2002.

[8] C Massin, F Vincent, A Homsy, K Ehrmann, G Boero, P-A Besse, A Daridon, E Verpoorte, NF De Rooij, and RS Popovic. Planar microcoil-based microfluidic nmr probes. Journal of Magnetic Resonance, 164(2):242-255, 2003.

[9] Henk Wensink, Fernando Benito-Lopez, Dorothee C Hermes, Willem Verboom, Han JGE Gardeniers, David N Reinhoudt, and Albert van den Berg. Measuring reaction kinetics in a lab-on-a-chip by microcoil nmr. Lab on a Chip, 5(3):280-284, 2005.

[10] Klaus Ehrmann, Nicolas Saillen, Franck Vincent, Matthieu Stettler, Martin Jordan, Florian Maria Wurm, Pierre-André Besse, and Radivoje Popovic. Microfabricated solenoids and helmholtz coils for nmr spectroscopy of mammalian cells. Lab on a Chip, 7(3):373-380, 2007.

[11] J Bart, JWG Janssen, PJM Van Bentum, APM Kentgens, and JGE Gardeniers. Optimization of stripline-based microfluidic chips for high-resolution nmr. Journal of Magnetic Resonance, 201(2):175-185, 2009.

[12] Jacob Bart, Ard J Kolkman, Anna Jo Oosthoek-de Vries, Kaspar Koch, Pieter J Nieuwland, Hans Janssen, Jan van Bentum, Kirsten AM Ampt, Floris PJT Rutjes, 
Sybren S Wijmenga, et al. A microfluidic high-resolution nmr flow probe. Journal of the American Chemical Society, 131(14):5014-5015, 2009.

[13] PJM Van Bentum, JWG Janssen, APM Kentgens, J Bart, and JGE Gardeniers. Stripline probes for nuclear magnetic resonance. Journal of Magnetic Resonance, 189(1):104-113, 2007.

[14] Oliver G Gruschke, Nicoleta Baxan, Lars Clad, Kai Kratt, Dominik von Elverfeldt, Andreas Peter, Jürgen Hennig, Vlad Badilita, Ulrike Wallrabe, and Jan G Korvink. Lab on a chip phased-array mr multi-platform analysis system. Lab on a Chip, 12 (3):495-502, 2012.

[15] Hans Georg Krojanski, Jrg Lambert, Yilmaz Gerikalan, Dieter Suter, and Roland Hergenrder. Microslot nmr probe for metabolomics studies. Analytical chemistry, 80(22):8668-8672, 2008.

[16] Marcel Utz and Reza Monazami. Nuclear magnetic resonance in microfluidic environments using inductively coupled radiofrequency resonators. Journal of Magnetic Resonance, 198(1):132-136, 2009.

[17] Jacques-François Jacquinot and Dimitris Sakellariou. Nmr signal detection using inductive coupling: Applications to rotating microcoils. Concepts in Magnetic Resonance Part A, 38(2):33-51, 2011.

[18] DI Hoult and RE Richards. Critical factors in the design of sensitive high resolution nuclear magnetic resonance spectrometers. Proceedings of the Royal Society of London. A. Mathematical and Physical Sciences, 344(1638):311-340, 1975. 
[19] David I Hoult and RE Richards. The signal-to-noise ratio of the nuclear magnetic resonance experiment. Journal of Magnetic Resonance (1969), 24(1):71-85, 1976.

[20] John A Sidles and Daniel Rugar. Signal-to-noise ratios in inductive and mechanical detection of magnetic resonance. Physical review letters, 70(22):3506-3509, 1993.

[21] David I Hoult. Sensitivity and power deposition in a high-field imaging experiment. Journal of Magnetic Resonance Imaging, 12(1):46-67, 2000.

[22] Nian Wu, Timothy L Peck, Andrew G Webb, Richard L Magin, and Jonathan V Sweedler. 1h-nmr spectroscopy on the nanoliter scale for static and online measurements. Analytical Chemistry, 66(22):3849-3857, 1994.

[23] Dean L Olson, Timothy L Peck, Andrew G Webb, Richard L Magin, and Jonathan V Sweedler. High-resolution microcoil 1h-nmr for mass-limited, nanoliter-volume samples. Science, 270(5244):1967-1970, 1995.

[24] Raluca M Fratila and Aldrik H Velders. Small-volume nuclear magnetic resonance spectroscopy. Annual Review of Analytical Chemistry, 4:227-249, 2011.

[25] Raju Subramanian, Wayne P Kelley, Philip D Floyd, Zhixin J Tan, Andrew G Webb, and Jonathan V Sweedler. A microcoil nmr probe for coupling microscale hplc with on-line nmr spectroscopy. Anal. Chem, 68:4431r5, 1996.

[26] Andrew M Wolters, Dimuthu A Jayawickrama, Andrew G Webb, and Jonathan V Sweedler. Nmr detection with multiple solenoidal microcoils for continuous-flow capillary electrophoresis. Chem. Rev, 99:3133-3152, 1999. 
[27] J Schewitz, K Pusecker, P Gfrörer, U Götz, L-H Tseng, K Albert, and E Bayer. Direct coupling of capillary electrophoresis and nuclear magnetic resonance spectroscopy for the identification of a dinucleotide. Chromatographia, 50(5-6):333-337, 1999.

[28] JD Trumbull, IK Glasgow, DJ Beebe, and RL Magin. Integrating microfabricated fluidic systems and nmr spectroscopy. Biomedical Engineering, IEEE Transactions on, 47(1):3-7, 2000.

[29] Ville-Veikko Telkki, Vladimir V Zhivonitko, Susanna Ahola, Kirill V Kovtunov, Jukka Jokisaari, and Igor V Koptyug. Microfluidic gas-flow imaging utilizing parahydrogen-induced polarization and remote-detection nmr. Angewandte Chemie International Edition, 49(45):8363-8366, 2010.

[30] Erin E McDonnell, SongI Han, Christian Hilty, Kimberly L Pierce, and Alexander Pines. Nmr analysis on microfluidic devices by remote detection. Analytical chemistry, 77(24):8109-8114, 2005.

[31] Elad Harel, Christian Hilty, Katherine Koen, Erin E McDonnell, and Alex Pines. Time-of-flight flow imaging of two-component flow inside a microfluidic chip. Physical review letters, 98(1):017601, 2007.

[32] DI Hoult and B Tomanek. Use of mutually inductive coupling in probe design. Concepts in magnetic resonance, 15(4):262-285, 2002.

[33] Mehmet Bilgen et al. Inductively-overcoupled coil design for high resolution magnetic resonance imaging. Biomed Eng Online, 5(3), 2006. 
[34] D Sakellariou, G Le Goff, and J-F Jacquinot. High-resolution, high-sensitivity nmr of nanolitre anisotropic samples by coil spinning. Nature, 447(7145):694-697, 2007.

[35] Jerome E Gurst. Nmr and the structure of d-glucose. Journal of Chemical Education, 68(12):1003, 1991.

[36] G Navon, YQ Song, T Room, S Appelt, RE Taylor, and A Pines. Enhancement of solution nmr and mri with laser-polarized xenon. SCIENCE-NEW YORK THEN WASHINGTON-, pages 1848-1850, 1996.

[37] Malcolm H Levitt. Spin dynamics. Wiley New York, 2001.

[38] Michael J Puddephat. Computer interface for convenient application for stereological methods for unbiased estimation of volume and surface area: studies using MRI with particular reference to the human brain. $\mathrm{PhD}$ thesis, University of Liverpool, 1999.

[39] N Hermanspahn, H Häffner, H-J Kluge, W Quint, S Stahl, J Verdú, and G Werth. Observation of the continuous stern-gerlach effect on an electron bound in an atomic ion. Physical review letters, 84(3):427-430, 2000.

[40] Thomas L. James. Biophysics textbook online: Fundamentals of nmr, chapter $1 @$ ONLINE, 2013. URL http://qudev.ethz.ch/content/courses/phys4/ studentspresentations/nmr/James_Fundamentals_of_NMR.pdf.

[41] James Keeler. Fourier transformation and data processing @ONLINE, 2013. URL http://www-keeler.ch.cam.ac.uk/lectures/Irvine/chapter4.pdf. 
[42] Jacob Bart. Stripline-based microfluidic devices for high-resolution NMR spectroscopy. University of Twente, 2009.

[43] C Connor. Advances in magnetic and optical resonance. Academic Press, Inc, 15: 201, 1990.

[44] Matthieu Guerquin-Kern. Wavelet-Based Reconstruction for Magnetic Resonance Imaging. PhD thesis, EPFL, 2012.

[45] DI Hoult and NS Ginsberg. The quantum origins of the free induction decay signal and spin noise. Journal of Magnetic Resonance, 148(1):182-199, 2001.

[46] JT Arnold, SS Dharmatti, and ME Packard. Chemical effects on nuclear induction signals from organic compounds. The Journal of Chemical Physics, 19(4):507-507, 1951.

[47] Charles P Slichter. Principles of magnetic resonance, volume 1. Springer, 1996.

[48] Arvin Moser. Philosophy to chemistry to elucidation @ONLINE, 2013. URL http://acdlabs.typepad.com/elucidation/cosytocsynoesyroesy/page/2/.

[49] Michael E Lacey, Raju Subramanian, Dean L Olson, Andrew G Webb, and Jonathan V Sweedler. High-resolution nmr spectroscopy of sample volumes from $1 \mathrm{nl}$ to $10 \mu \mathrm{l}$. Chem. Rev, 99(10):3133-3152, 1999.

[50] DI Hoult. The origins and present status of the radio wave controversy in $\mathrm{nmr}$. Concepts in Magnetic Resonance Part A, 34(4):193-216, 2009.

[51] Olaf Beckonert, Hector C Keun, Timothy MD Ebbels, Jacob Bundy, Elaine Holmes, John C Lindon, and Jeremy K Nicholson. Metabolic profiling, 
metabolomic and metabonomic procedures for nmr spectroscopy of urine, plasma, serum and tissue extracts. Nature protocols, 2(11):2692-2703, 2007.

[52] John C Lindon and Jeremy K Nicholson. Spectroscopic and statistical techniques for information recovery in metabonomics and metabolomics. Annu. Rev. Anal. Chem., 1:45-69, 2008.

[53] Zhengzheng Pan and Daniel Raftery. Comparing and combining nmr spectroscopy and mass spectrometry in metabolomics. Analytical and bioanalytical chemistry, $387(2): 525-527,2007$.

[54] Andrea Amantonico, Pawel L Urban, and Renato Zenobi. Analytical techniques for single-cell metabolomics: state of the art and trends. Analytical and bioanalytical chemistry, 398(6):2493-2504, 2010.

[55] Anatole Abragam. The principles of nuclear magnetism, volume 32. Oxford University Press, 1961.

[56] Albert W Overhauser. Polarization of nuclei in metals. Physical Review, 92(2): $411,1953$.

[57] Jan H Ardenkjær-Larsen, Björn Fridlund, Andreas Gram, Georg Hansson, Lennart Hansson, Mathilde H Lerche, Rolf Servin, Mikkel Thaning, and Klaes Golman. Increase in signal-to-noise ratio of ; 10,000 times in liquid-state nmr. Proceedings of the National Academy of Sciences, 100(18):10158-10163, 2003.

[58] R Kaptein. Chemically induced dynamic nuclear polarization in five alkyl radicals. Chemical Physics Letters, 2(4):261-267, 1968. 
[59] Ralph W Adams, Juan A Aguilar, Kevin D Atkinson, Michael J Cowley, Paul IP Elliott, Simon B Duckett, Gary GR Green, Iman G Khazal, Joaquín LópezSerrano, and David C Williamson. Reversible interactions with para-hydrogen enhance nmr sensitivity by polarization transfer. Science, 323(5922):1708-1711, 2009.

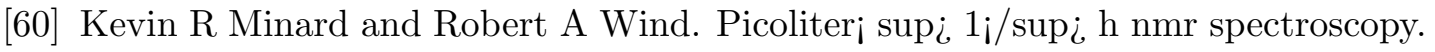
Journal of Magnetic Resonance, 154(2):336-343, 2002.

[61] Harald Günther. NMR spectroscopy: basic principles, concepts, and applications in chemistry. Wiley, 1992.

[62] Richard R Ernst, Geoffrey Bodenhausen, Alexander Wokaun, et al. Principles of nuclear magnetic resonance in one and two dimensions, volume 14. Clarendon Press Oxford, 1987.

[63] Edwin D Becker. High resolution NMR: theory and chemical applications. Academic Press, 1999.

[64] Vlad Badilita, Robert Ch Meier, Nils Spengler, Ulrike Wallrabe, Marcel Utz, and Jan G Korvink. Microscale nuclear magnetic resonance: a tool for soft matter research. Soft Matter, 8(41):10583-10597, 2012.

[65] AG Webb and SC Grant. Signal-to-noise and magnetic susceptibility trade-offs in solenoidal microcoils for nmr. Journal of magnetic resonance. Series B, 113(1):83, 1996.

[66] K Yamauchi, JWG Janssen, and APM Kentgens. Implementing solenoid microcoils for wide-line solid-state nmr. Journal of Magnetic Resonance, 167(1):87-96, 2004. 
[67] Kevin R Minard and Robert A Wind. Solenoidal microcoil design. part i: Optimizing rf homogeneity and coil dimensions. Concepts in Magnetic Resonance, 13 (2):128-142, 2001.

[68] Kevin R Minard and Robert A Wind. Solenoidal microcoil design. part ii: Optimizing winding parameters for maximum signal-to-noise performance. Concepts in Magnetic Resonance, 13(3):190-210, 2001.

[69] Frank Engelke. Electromagnetic wave compression and radio frequency homogeneity in nmr solenoidal coils: computational approach. Concepts in Magnetic Resonance, 15(2):129-155, 2002.

[70] Xiaoliang Zhang, Kamil Ugurbil, and Wei Chen. Microstrip rf surface coil design for extremely high-field mri and spectroscopy. Magnetic resonance in medicine, 46(3):443-450, 2001.

[71] Krzysztof Jasiński, Anna Młynarczyk, Peter Latta, Vyacheslav Volotovskyy, Władyslaw P Węglarz, and Bogusław Tomanek. A volume microstrip rf coil for mri microscopy. Magnetic Resonance Imaging, 30(1):70-77, 2012.

[72] Yael Maguire, Isaac L Chuang, Shuguang Zhang, and Neil Gershenfeld. Ultrasmall-sample molecular structure detection using microslot waveguide nuclear spin resonance. Proceedings of the National Academy of Sciences, 104(22):9198-9203, 2007.

[73] Herbert Ryan, Suk-Heung Song, Anja Zaß, Jan Korvink, and Marcel Utz. Contactless nmr spectroscopy on a chip. Analytical chemistry, 84(8):3696-3702, 2012. 
[74] David J Beebe, Jonathan D Trumbull, and Ian K Glasgow. Microfluidics and bioanalysis systems: issues and examples. In Engineering in Medicine and Biology Society, 1998. Proceedings of the 20th Annual International Conference of the IEEE, volume 4, pages 1692-1697. IEEE, 1998.

[75] Klaus Ehrmann, Kristopher Pataky, Matthieu Stettler, Florian Maria Wurm, Jürgen Brugger, Pierre-André Besse, and Radivoje Popovic. Nmr spectroscopy and perfusion of mammalian cells using surface microprobes. Lab on a Chip, 7(3): 381-383, 2007.

[76] Stefan Leidich, Marco Braun, Thomas Gessner, and Thomas Riemer. Silicon cylinder spiral coil for nuclear magnetic resonance spectroscopy of nanoliter samples. Concepts in Magnetic Resonance Part B: Magnetic Resonance Engineering, 35(1): $11-22,2009$.

[77] Curtis D Chin, Vincent Linder, and Samuel K Sia. Commercialization of microfluidic point-of-care diagnostic devices. Lab on a Chip, 12(12):2118-2134, 2012.

[78] Chong H Ahn, Jin-Woo Choi, Gregory Beaucage, Joseph H Nevin, Jeong-Bong Lee, Aniruddha Puntambekar, and Jae Y Lee. Disposable smart lab on a chip for point-of-care clinical diagnostics. Proceedings of the IEEE, 92(1):154-173, 2004.

[79] Anna J Tüdős, Geert AJ Besselink, and Richard BM Schasfoort. Trends in miniaturized total analysis systems for point-of-care testing in clinical chemistry. Lab on a Chip, 1(2):83-95, 2001.

[80] Anja Zaß. Self-resonant, inductively coupled microcoils for $\mathrm{nmr}$ spectroscopy on a chip. Master's thesis, University of Freiburg, 2011. 
[81] System Overview, Pub. No. 01-999261-00, Rev. A0305. Varian, Inc. NMR Systems, .

[82] High0Field Triple Resonance Probe Installation. Varian, Inc. NMR Systems, .

[83] Timothy L Peck, Richard L Magin, and Paul C Lauterbur. Design and analysis of microcoils for nmr microscopy. Journal of Magnetic Resonance, Series B, 108 (2):114-124, 1995.

[84] Sertac Eroglu, Barjor Gimi, Brian Roman, Gary Friedman, and Richard L Magin. Nmr spiral surface microcoils: design, fabrication, and imaging. Concepts in Magnetic Resonance Part B: Magnetic Resonance Engineering, 17(1):1-10, 2003.

[85] Dean L Olson, Michael E Lacey, and Jonathan V Sweedler. High-resolution microcoil nmr for analysis of mass-limited, nanoliter samples. Analytical chemistry, 70(3):645-650, 1998.

[86] David R Lide and Thomas J Bruno. CRC handbook of chemistry and physics. CRC PressI Llc, 2012.

[87] F David Doty, George Entzminger, and Y Andy Yang. Magnetism in highresolution nmr probe design. i: General methods. Concepts in Magnetic Resonance, 10(3):133-156, 1998.

[88] Andrew G. Webb. Radiofrequency microcoils in magnetic resonance. Progress in Nuclear Magnetic Resonance Spectroscopy, 31(1):1-42, 1997.

[89] Vlad Badilita, Kai Kratt, Nicoleta Baxan, Mohammad Mohmmadzadeh, Tobias Burger, Hans Weber, Dominik v Elverfeldt, Jürgen Hennig, Jan G Korvink, and 
Ulrike Wallrabe. On-chip three dimensional microcoils for mri at the microscale. Lab on a Chip, 10(11):1387-1390, 2010.

[90] ET Enikov and BJ Nelson. Three-dimensional microfabrication for a multi-degreeof-freedom capacitive force sensor using fibre-chip coupling. Journal of Micromechanics and Microengineering, 10(4):492, 2000.

[91] Zhixiong Xiao, Wuyong Peng, RF Wolffenbuttel, and KR Farmer. Micromachined variable capacitors with wide tuning range. Sensors and Actuators A: Physical, 104(3):299-305, 2003.

[92] Harold A Wheeler. Simple inductance formulas for radio coils. Proceedings of the Institute of Radio Engineers, 16(10):1398-1400, 1928.

[93] Sunderarajan S Mohan, Maria del Mar Hershenson, Stephen P Boyd, and Thomas H Lee. Simple accurate expressions for planar spiral inductances. SolidState Circuits, IEEE Journal of, 34(10):1419-1424, 1999.

[94] Carmen R Reedy, Carol W Price, Jeff Sniegowski, Jerome P Ferrance, Matthew Begley, and James P Landers. Solid phase extraction of dna from biological samples in a post-based, high surface area poly (methyl methacrylate)(pmma) microdevice. Lab on a Chip, 11(9):1603-1611, 2011.

[95] Wenhua Zhang, Shuichao Lin, Chunming Wang, Jia Hu, Cong Li, Zhixia Zhuang, Yongliang Zhou, Richard A Mathies, and Chaoyong James Yang. Pmma/pdms valves and pumps for disposable microfluidics. Lab on a Chip, 9(21):3088-3094, 2009. 
[96] Ray-Hua Horng, Pin Han, Hung-Yu Chen, Kuan-Wen Lin, Tung-Mung Tsai, and Jyh-Myng Zen. Pmma-based capillary electrophoresis electrochemical detection microchip fabrication. Journal of Micromechanics and Microengineering, 15(1):6, 2005.

[97] Jenny A Lounsbury, Brian L Poe, Michael Do, and James P Landers. Laserablated poly (methyl methacrylate) microdevices for sub-microliter dna amplification suitable for micro-total analysis systems. Journal of Micromechanics and Microengineering, 22(8):085006, 2012.

[98] Che-Hsin Lin, Chien-Hsiang Chao, and Che-Wei Lan. Low azeotropic solvent for bonding of pmma microfluidic devices. Sensors and Actuators B: chemical, 121 (2):698-705, 2007.

[99] F David Doty, George Entzminger Jr, and Cory D Hauck. Error-tolerant rf litz coils for nmr/mri. Journal of Magnetic Resonance, 140(1):17-31, 1999.

[100] L Renaud, M Armenean, L Berry, P Kleimann, P Morin, M Pitaval, J O'Brien, M Brunet, and H Saint-Jalmes. Implantable planar rf microcoils for nmr microspectroscopy. Sensors and Actuators A: Physical, 99(3):244-248, 2002. 